\title{
Electrochemical impedance spectroscopy of proton exchange membrane fuel cell stacks
}

\author{
Brian J. Hetzer \\ West Virginia University
}

Follow this and additional works at: https://researchrepository.wvu.edu/etd

\section{Recommended Citation}

Hetzer, Brian J., "Electrochemical impedance spectroscopy of proton exchange membrane fuel cell stacks" (2004). Graduate Theses, Dissertations, and Problem Reports. 1492.

https://researchrepository.wvu.edu/etd/1492

This Thesis is protected by copyright and/or related rights. It has been brought to you by the The Research Repository @ WVU with permission from the rights-holder(s). You are free to use this Thesis in any way that is permitted by the copyright and related rights legislation that applies to your use. For other uses you must obtain permission from the rights-holder(s) directly, unless additional rights are indicated by a Creative Commons license in the record and/ or on the work itself. This Thesis has been accepted for inclusion in WVU Graduate Theses, Dissertations, and Problem Reports collection by an authorized administrator of The Research Repository @ WVU. For more information, please contact researchrepository@mail.wvu.edu. 


\title{
Electrochemical Impedance Spectroscopy of Proton Exchange Membrane Fuel Cell Stacks
}

\author{
Brian J. Hetzer \\ Thesis submitted to the \\ College of Engineering and Mineral Resources \\ at West Virginia University \\ in partial fulfillment of the requirements \\ for the degree of \\ Master of Science \\ in \\ Electrical Engineering
}

\author{
Roy S. Nutter Jr., Ph.D., Chair \\ Wils L. Cooley, Ph.D. \\ Randall S. Gemmen, Ph.D. \\ Mark A. Jerabek, Ph.D. \\ Lane Department of Computer Science \\ and Electrical Engineering
}
Morgantown, West Virginia
2004

Keywords: EIS, Impedance Response, PEM, PEMFC, Model

Copyright 2004 Brian J. Hetzer 


\title{
ABSTRACT \\ Electrochemical Impedance Spectroscopy of Proton Exchange Membrane Fuel Cell Stacks
}

\author{
Brian J. Hetzer
}

Electrochemical Impedance Spectroscopy (EIS) is applied to a small, four cell Proton Exchange Membrane (PEM) fuel cell stack to examine the stack response as the load current changes. The measured stack response is compared to a simulated response generated by a stack model that has been developed using MATLAB. The results show that the model predicted well the behavior of the VI curve for currents less than 50 Amps. The model also qualitatively predicted the impedance response as a function of current and frequency. Because of apparent model incompleteness, it was not able to predict accurately the shape of the response in the complex plane at currents larger than 20 Amps and did not match the change in ohmic resistance with current. 


\section{Dedication}

This paper is dedicated to all those who have helped me reach this point in my life. My family and friends have provided me with many of the opportunities that gave me the chance to pursue this goal and a lot of support needed to accomplish this work. I would like to specifically mention my twin brother, Michael, who has been a great source of encouragement and healthy competition throughout my life. I am especially grateful to my loving wife and best friend, Laura, who has been a great source of strength and encouragement to me despite the many long and lonely nights she faced. 


\section{Acknowledgment}

I would like to acknowledge my advisor, Dr. Roy Nutter, who has been instrumental in helping me throughout the development of my thesis. I am also grateful to the other members of my committee, Dr. Wils Cooley, Dr. Randall Gemmen, and Dr. Mark Jerabek for their help and support. I also need to acknowledge the U.S. Department of Energy, National Energy Technology Laboratory, and all those who work in the MAC Lab for their assistance and hard work that went into developing this thesis. 


\section{Table of Contents}

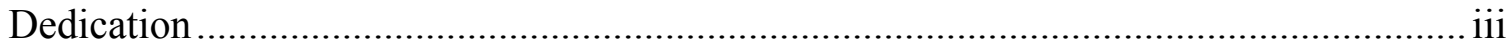

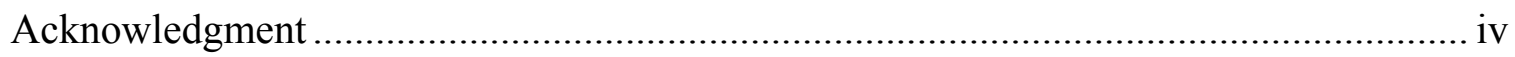

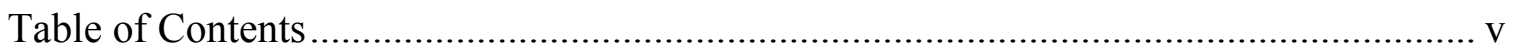

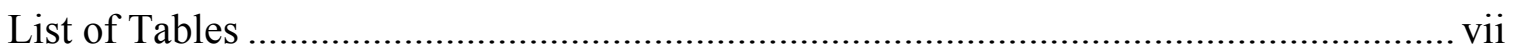

List of Figures ................................................................................................... viii

List of Abbreviations, Symbols, or Nomenclature ………………………................... $\mathrm{x}$

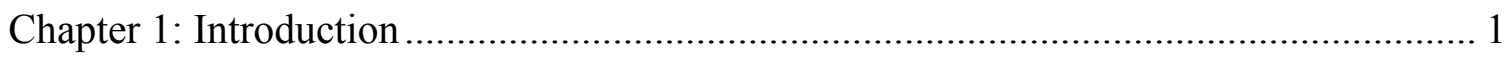

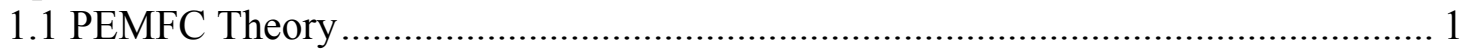

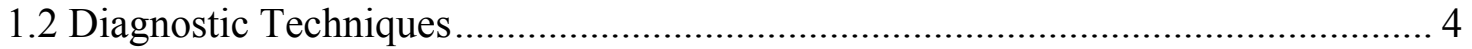

1.2.1 Survey of Diagnostic Techniques ................................................................ 5

1.2.2 Electrochemical Impedance Spectroscopy (EIS) ........................................... 8

1.2.3 Interpretation of EIS Measurements .......................................................... 9

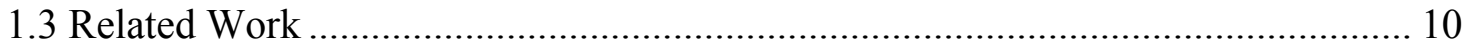

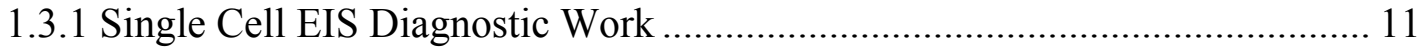

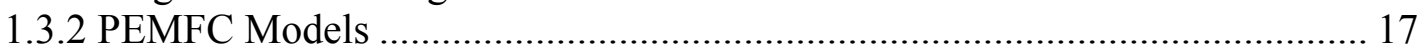

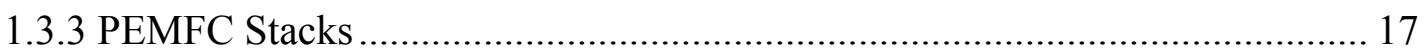

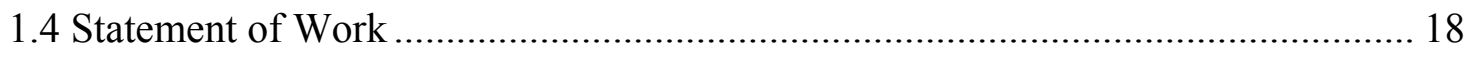

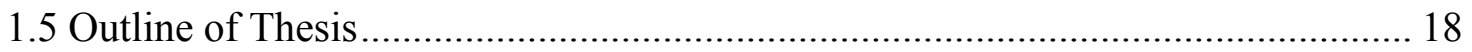

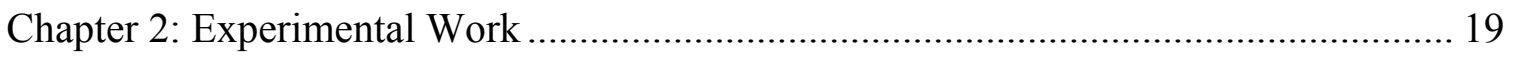

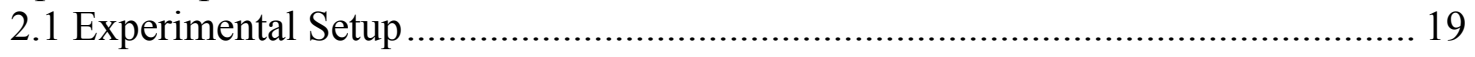

2.1.1 PEM Fuel Cell....................................................................................... 19

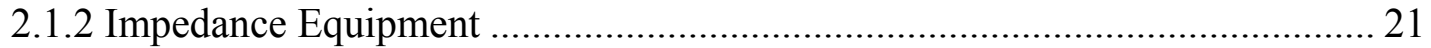

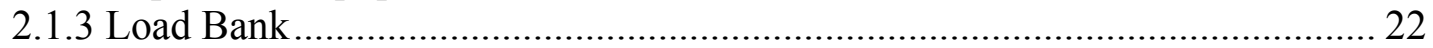

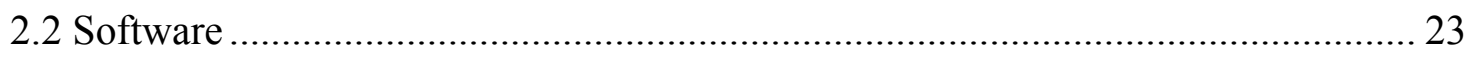

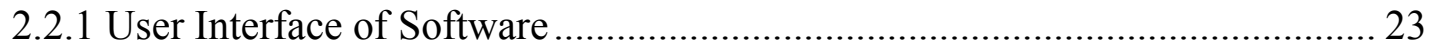

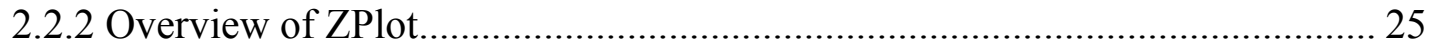

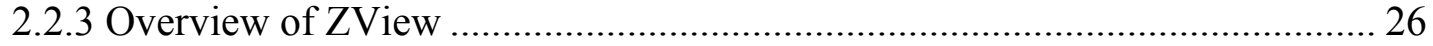

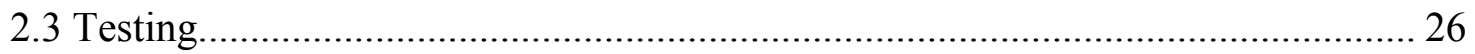

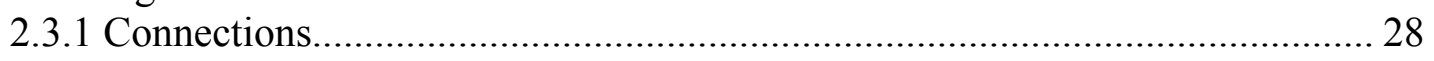

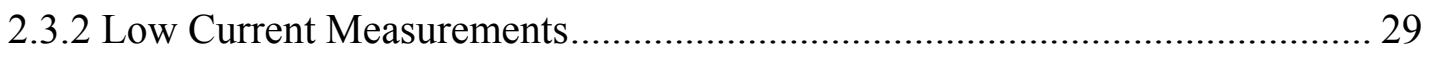

2.3.3 High Current Measurements ............................................................................. 30

2.3.4 Parallel Load Effects.................................................................................. 32

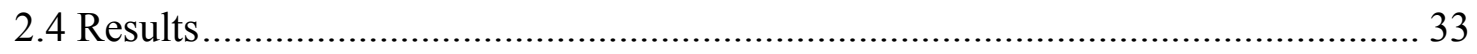

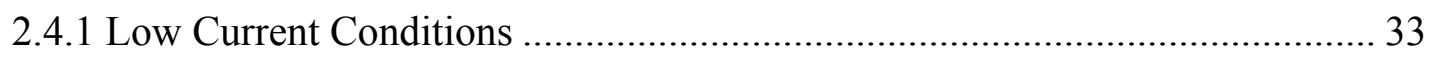

2.4.2 High Current Condition .............................................................................. 35 


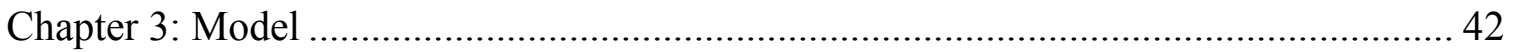

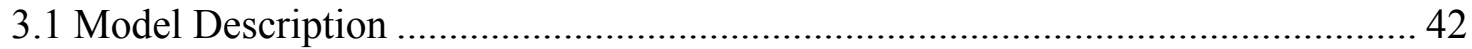

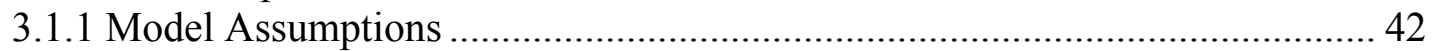

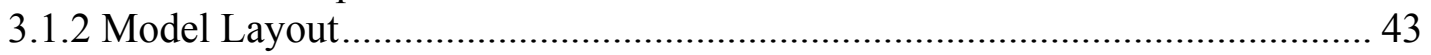

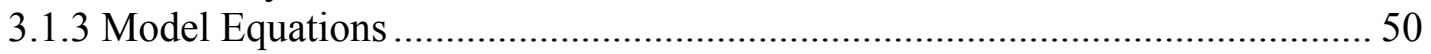

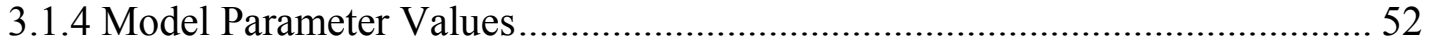

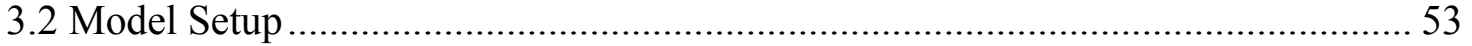

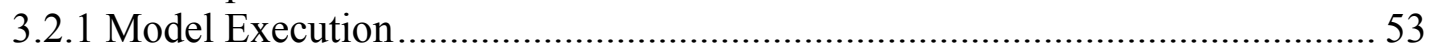

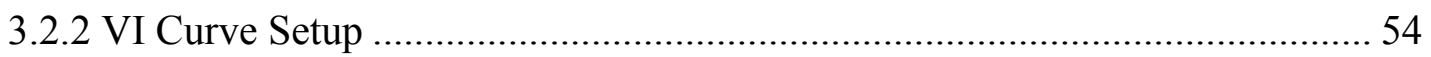

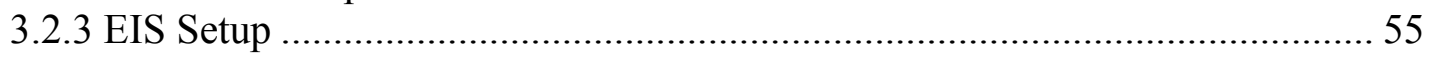

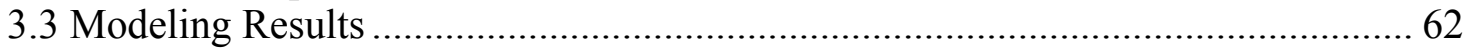

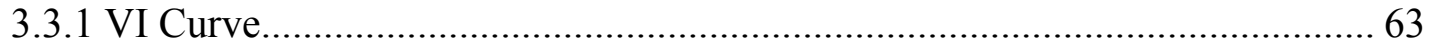

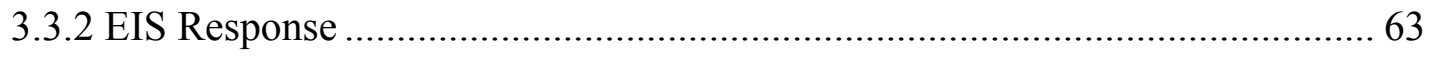

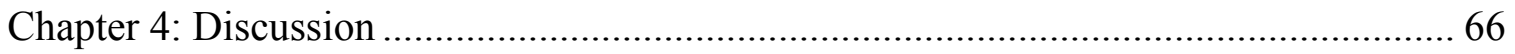

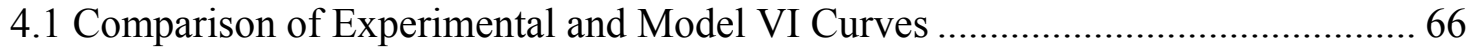

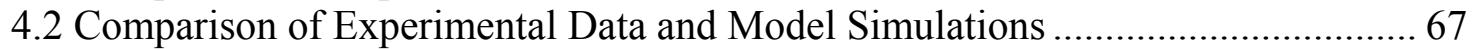

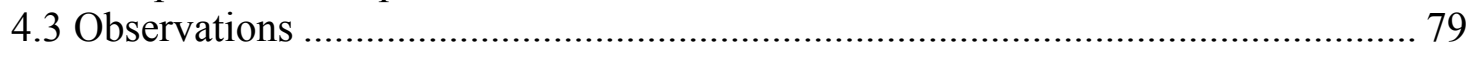

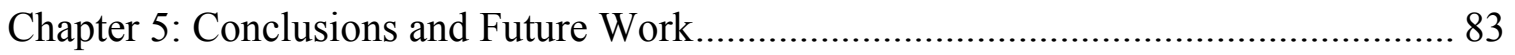

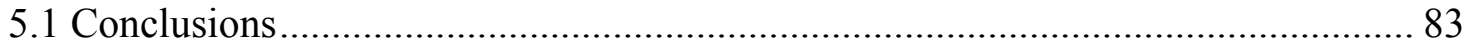

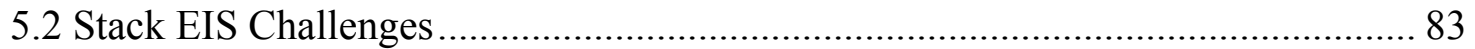

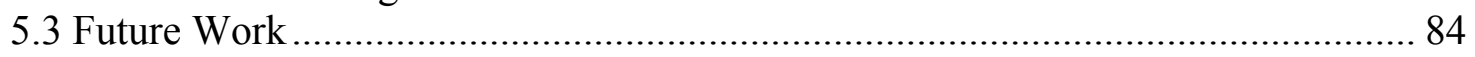

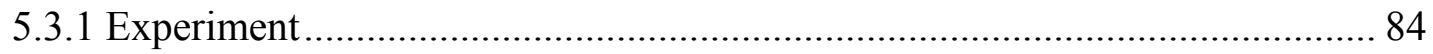

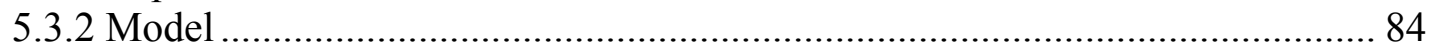

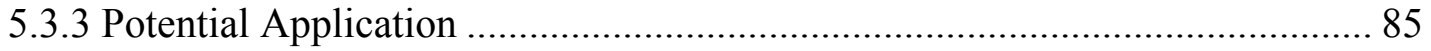

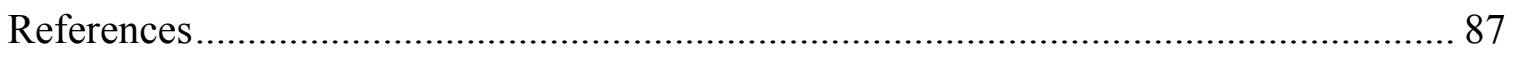

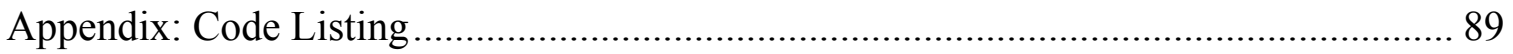




\section{List of Tables}

Table 2.1: Comparison of Current Setting and Actual Current ......................................... 32

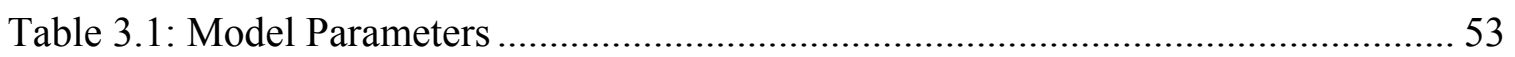

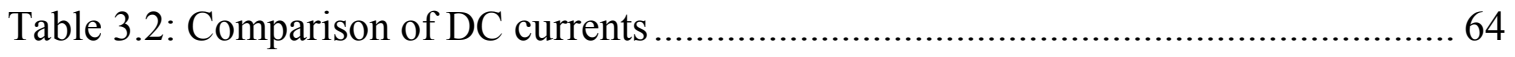

Table A.1: Assignment of Setup File Variables ……………………………………....... 95 


\section{List of Figures}

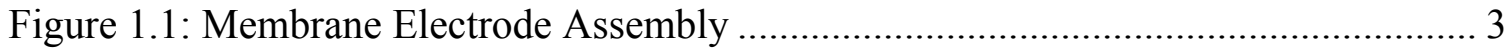

Figure 1.2: Sample VI Polarization Curve ............................................................... 5

Figure 1.3: Sample CI Curve ..................................................................................... 7

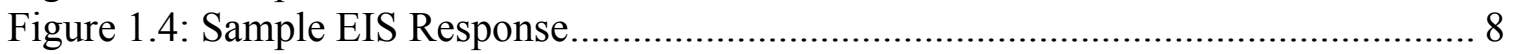

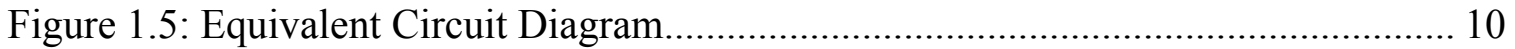

Figure 1.6: Parthasarathy et al. Results of Cathode Response at Potentials $0.8 \mathrm{~V}$ to $0.95 \mathrm{~V}$

Figure 1.7: Parthasarathy et al. Results of Cathode Response at Potentials $0.35 \mathrm{~V}$ to $0.75 \mathrm{~V}$

Figure 1.8: Springer et al. Results for Air Cathode ................................................ 13

Figure 1.9: Paganin et al. Results for Oxygen Cathode.................................................. 14

Figure 1.10: General Trend of EIS Response with Current............................................ 16

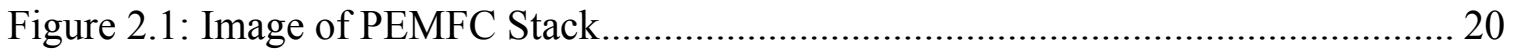

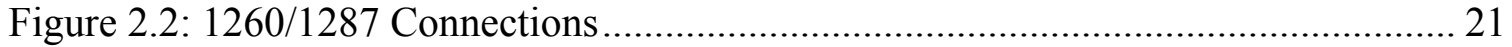

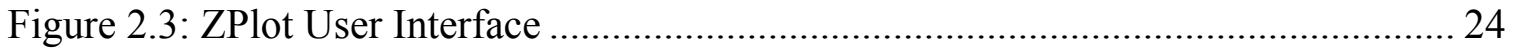

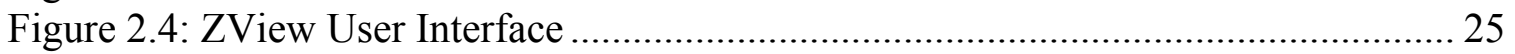

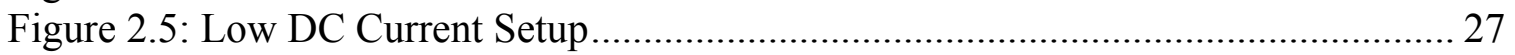

Figure 2.6: High DC Current Setup ......................................................................... 28

Figure 2.7: Two-Terminal Connection to PEM Fuel Cell using Solartron 1287............. 29

Figure 2.8: Typical VI curve for the PEMFC stack............................................... 31

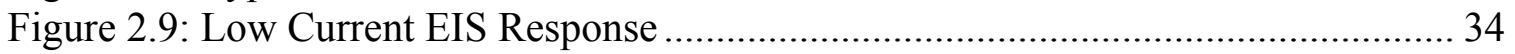

Figure 2.10: Bode Plot of Low Current Response ..................................................... 34

Figure 2.11: High Frequency of Low Current EIS Response ....................................... 35

Figure 2.12: Effect of Changing AC Magnitude at 20 Amps Load................................. 36

Figure 2.13: Measured Total Impedance Response ...................................................... 38

Figure 2.14: Impedance Response of Avtron Load at Various Currents (Inset: Close up of

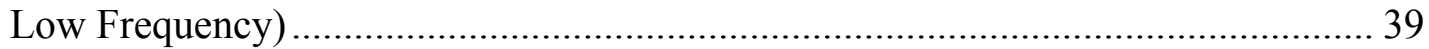

Figure 2.15: Load Correction Applied to 20A Measurement ........................................ 40

Figure 2.16: Corrected PEMFC Stack Response .......................................................... 41

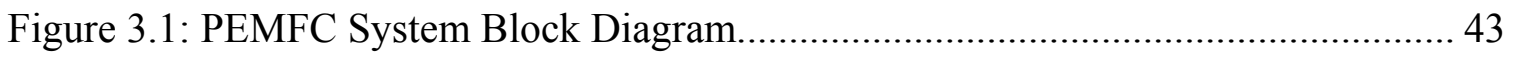

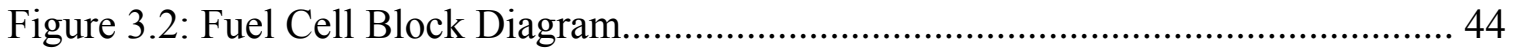

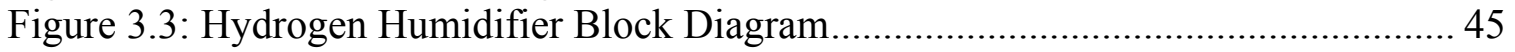

Figure 3.4: Air Humidifier Block Diagram .............................................................. 45

Figure 3.5: Individual Cell Block Diagram .................................................................. 46

Figure 3.6: Anode Hydrogen Conservation Block Diagram .......................................... 46

Figure 3.7: Cathode Oxygen / Water Conservation Block Diagram ............................. 47

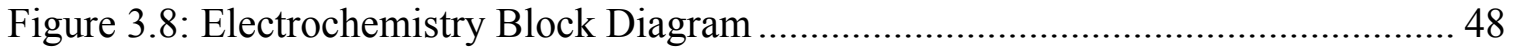

Figure 3.9: Activation Overpotential Calculation Block Diagram ................................ 49

Figure 3.10: Anode $\mathrm{H}_{2}$ Diffusion Gradient Block Diagram ......................................... 49

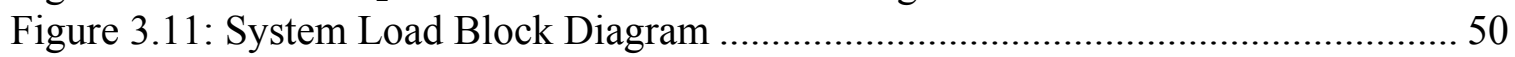

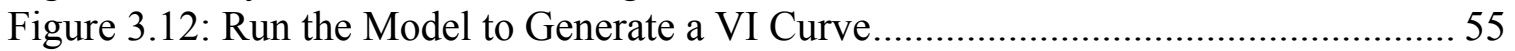

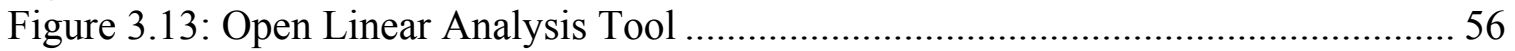




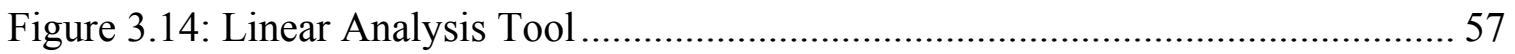

Figure 3.15: Placing Input and Output Points............................................................. 58

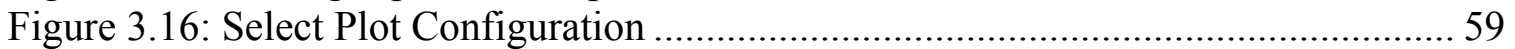

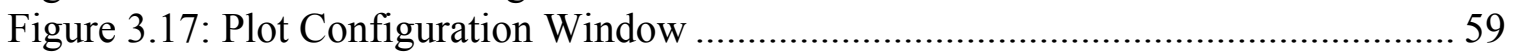

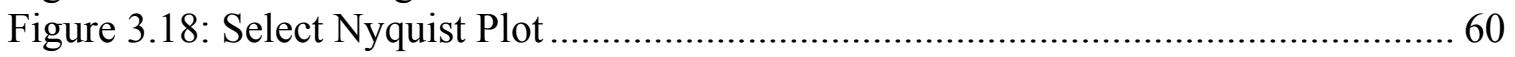

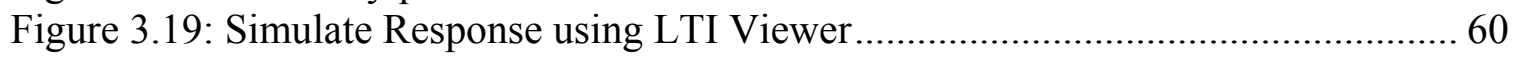

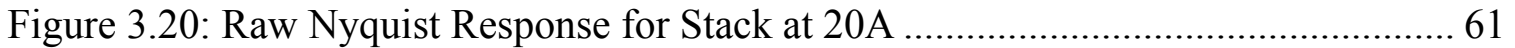

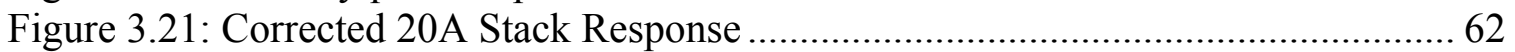

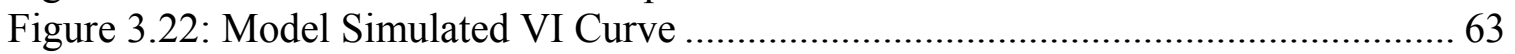

Figure 3.23: Simulated EIS Responses of the PEMFC Stack........................................... 64

Figure 3.24: High Current Simulated EIS Responses of the PEMFC Stack .................... 65

Figure 4.1: Comparison of Experimental and Model VI Curves...................................... 66

Figure 4.2: Comparison of Experimental Data and Model Simulation for Stack PEMFC at

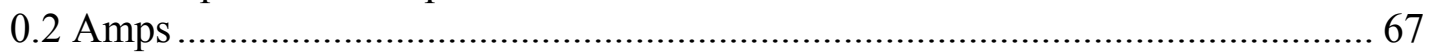

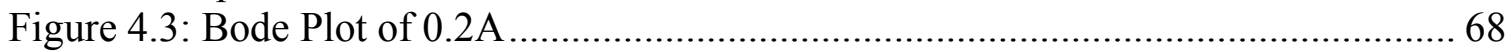

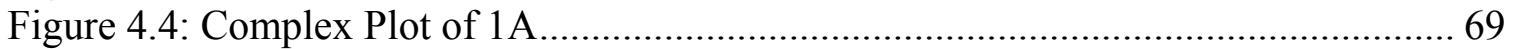

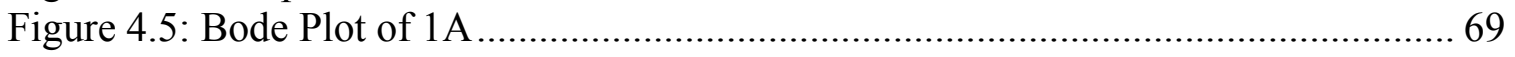

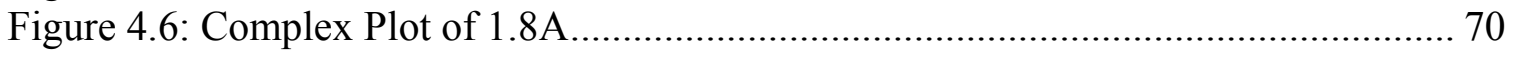

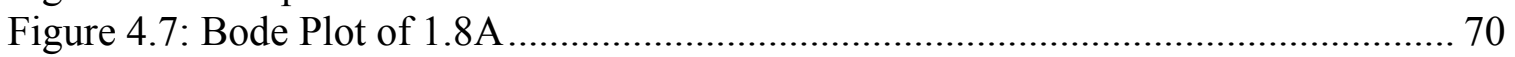

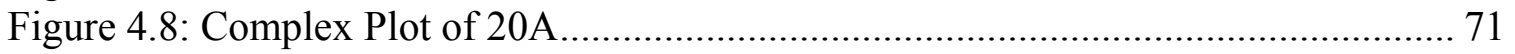

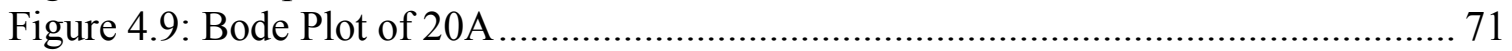

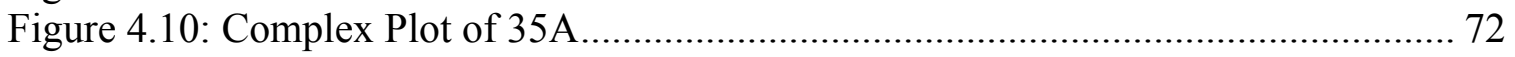

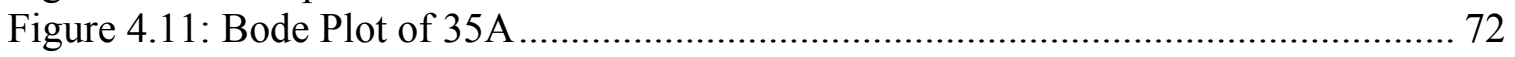

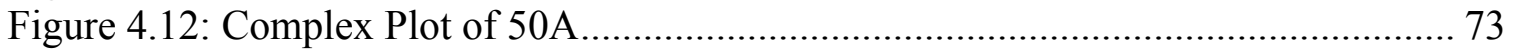

Figure 4.13: Bode Plot of 50A .......................................................................... 73

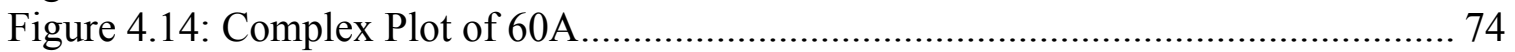

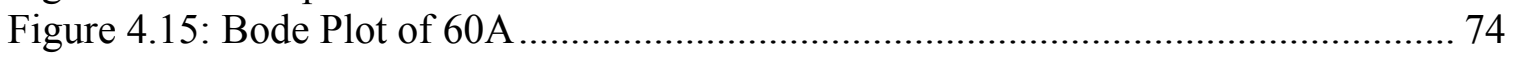

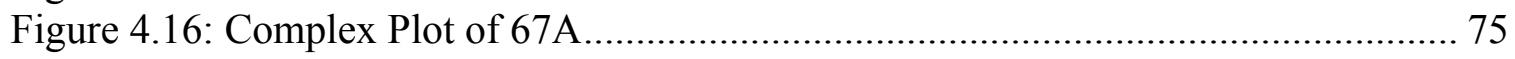

Figure 4.17: Bode Plot of 67A ............................................................................... 75

Figure 4.18: 3D Bode Magnitude of all Experimental PEMFC Stack Measurements ..... 77

Figure 4.19: 3D Bode Magnitude of all PEMFC Stack Model Simulations ..................... 77

Figure 4.20: 3D Bode Phase of all Experimental PEMFC Stack Measurements ............. 78

Figure 4.21: 3D Bode Phase of all PEMFC Stack Model Simulations …………............ 78

Figure 4.22: Effect of Current on Ohmic Resistance of Fuel Cell ................................... 81

Figure 5.1: Simple Fuel Cell Stack Equivalent Circuit................................................ 85

Figure 5.2: Responses of Simple Stack Example ……………..................................... 86

Figure 5.3: Bode Plot of Simple Stack Example ……………........................................ 86 


\title{
List of Abbreviations, Symbols, or Nomenclature
}

\author{
ABBREVIATIONS \\ AC Alternating Current \\ $\mathrm{CC} \quad$ Constant Current \\ CE Counter Electrode \\ CV Constant Voltage \\ DC Direct Current \\ ECI Electrochemical Interface \\ EIS Electrochemical Impedance Spectroscopy \\ FRA Frequency Response Analyzer \\ HOR Hydrogen Oxidation Reaction \\ MEA Membrane Electrode Assembly \\ OCP Open Circuit Potential \\ ORR Oxygen Reduction Reaction \\ PEMFC Proton Exchange Membrane Fuel Cell \\ RE1 Reference Electrode 1 \\ RE2 Reference Electrode 2 \\ rms root mean square \\ WE Working Electrode
}

NOMENCLATURE

$\begin{array}{ll}\mathrm{A}_{\text {cell }} & \text { Active Area of Individual Fuel Cell }\left[\mathrm{cm}^{2}\right] \\ \mathrm{C} & \left.\text { Concentration [mol } / \mathrm{m}^{3}\right] \\ \mathrm{C}^{\mathrm{fs}} & \text { Free Stream Concentration }\left[\mathrm{mol} / \mathrm{m}^{3}\right] \\ C_{H_{2}} & \left.\text { Hydrogen Concentration [mol } / \mathrm{m}^{3}\right] \\ C_{H_{2}}^{r e f} & \text { Reference Hydrogen Concentration }\left(25.52 \mathrm{~mol} / \mathrm{m}^{3}\right) \\ C_{O_{2}} & \left.\text { Oxygen Concentration [mol } / \mathrm{m}^{3}\right] \\ C_{O_{2}}^{r e f} & \text { Reference Oxygen Concentration }\left(4.623 \mathrm{~mol} / \mathrm{m}^{3}\right) \\ \mathrm{C}_{\mathrm{DL}} & \text { Capacitance of Double Layer }\left[\mathrm{Farad} / \mathrm{cm}^{2}\right] \\ \mathrm{D}^{\prime} & \text { Effective Diffusion Coefficient }\left[\mathrm{m}^{2} / \mathrm{sec}\right] \\ D_{H_{2}} & \text { Hydrogen Diffusion Coefficient }\left[\mathrm{m}^{2} / \mathrm{sec}\right] \\ D_{O_{2}} & \text { Oxygen Diffusion Coefficient }\left[\mathrm{m}^{2} / \mathrm{sec}\right] \\ \mathrm{E} & \text { Cell Potential [Volts] } \\ \mathrm{E}_{\mathrm{N}} & \text { Nernst Potential [Volts] } \\ \mathrm{E}_{\mathrm{o}} & \text { Standard State Potential [Volts] } \\ \mathrm{f} & \text { Frequency [Hz] } \\ \mathrm{F} & \text { Faraday's Constant (96,439 Coulomb } / \mathrm{gram}-\mathrm{mol}) \\ \mathrm{i} & \left.\text { Current Density [Amp/cm }{ }^{2}\right] \\ \mathrm{i}_{\text {diff }} & \text { Diffusion Limited Current Density }\left[\text { Amp } / \mathrm{cm}^{2}\right] \\ \mathrm{i}_{\mathrm{o}} & \left.\text { Exchange Current Density [Amp } / \mathrm{cm}{ }^{2}\right]\end{array}$




$\begin{array}{ll}i_{o}^{r e f} & \text { Reference Exchange Current Density }\left(0.0017 \mathrm{Amp} / \mathrm{cm}^{2}\right) \\ \mathrm{p} & \text { Pressure [Pascal] } \\ \mathrm{P}_{\text {electrical }} & \text { Electrical Power [Watts] } \\ p_{H_{2}} & \text { Partial Pressure of Hydrogen [Pascal] } \\ p_{H_{2}}^{r e f} & \text { Reference Partial Pressure of Hydrogen (101,000 Pascal) } \\ p_{H_{2} O} & \text { Partial Pressure of Water Vapor [Pascal] } \\ p_{O_{2}} & \text { Partial Pressure of Oxygen [Pascal] } \\ p_{O_{2}}^{r e f} & \text { Reference Partial Pressure of Oxygen (101,000 Pascal) } \\ \mathrm{p}_{\mathrm{o}} & \text { Reference Standard State Pressure [Pascal] } \\ \mathrm{R} & \text { Universal Gas Constant }(8.3144 \text { Joule/gram-mol-Kelvin }) \\ \mathrm{r}_{\text {ohmic }} & \left.\text { Area Specific Ohmic Resistance [ } \Omega \text {-cm }{ }^{2}\right] \\ \mathrm{T} & \text { Temperature [Kelvin] } \\ \mathrm{T}_{\text {ref }} & \text { Reference Temperature (353 Kelvin) } \\ \mathrm{V} & \text { Voltage [Volts] } \\ \mathrm{Z} & \text { Impedance }\left[\Omega \text {-cm }{ }^{2}\right] \\ \mathrm{Z} & \text { Number of Electrons }\end{array}$

\section{GREEK}

$\begin{array}{ll}\alpha & \text { Transfer Coefficient }(0.5) \\ \delta & \text { Diffusion Layer Thickness (0.0004 m) } \\ \varepsilon & \text { Diffusion Layer Porosity (0.4) } \\ \eta & \text { Overpotential [Volts] } \\ \eta_{\text {act }} & \text { Activation Overpotential [Volts] } \\ \eta_{d i f f}^{a} & \text { Anode Diffusion Overpotential [Volts] } \\ \eta_{d i f f}^{c} & \text { Cathode Diffusion Overpotential [Volts] } \\ \tau & \text { Diffusion Layer Tortuosity (4) } \\ \omega & \text { Angular Frequency [rad/sec] } \\ \omega_{\max } & \text { Angular Frequency at Maximum of EIS Response [rad/sec] }\end{array}$




\section{Chapter 1: Introduction}

Research into Proton Exchange Membrane Fuel Cells (PEMFCs) has been ongoing for many decades. Much of this research has been motivated by growing concerns over energy usage and availability. With oil supplies shrinking and energy demands rapidly growing, there exists a need for an alternative, more efficient, means of supplying our energy demands [1]. A PEMFC is just such a device. A PEMFC is a simple device which takes hydrogen and oxygen gases and combines them through a chemical reaction to produce water and electricity. The power output of a practical PEMFC can range anywhere from a few watts to hundreds of kilowatts [2]. The primary applications of PEMFCs are in the transportation sector as a replacement for the Internal Combustion Engine, and in the power generation sector as a local, on- or off-grid power source. A PEMFC powered vehicle would require approximately a 40-60 kW [3] fuel cell for a passenger car, whereas a public transportation bus would require a peak power of up to $150-250 \mathrm{~kW}$ [4]. If used to power a single family house, a $5 \mathrm{~kW}$ PEMFC, with appropriate energy storage devices to handle peak demand, would be adequate [5]. When pure hydrogen is available as a fuel source, the PEMFC, as opposed to other types of fuel cells, is best suited for the automotive sector because of its relatively low operating temperature, quick response time and high power density. Since the technology is not yet fully mature, there is still the need to have advanced diagnostics to better understand how a PEMFC operates, particularly when arranged into a stack, where cells are connected serially to increase the power output.

\subsection{PEMFC Theory}

A PEMFC works by directly converting hydrogen gas into water and energy through a direct electrochemical process. Conventionally, energy is obtained from the heat generated by combustion of a hydrocarbon fuel. This is the process used in all internal combustion engines, turbines, boilers, etc., in which the chemical energy in the fuel is released as heat through combustion processes. The heat is used to perform some mechanical work (i.e., push a piston or spin a turbine) which is then converted to 
electrical work through a generator. The advantage in a PEMFC is that it utilizes a direct process for converting the fuel's chemical energy into electrical energy, without the intermediate combustion step. The result is fewer losses and higher efficiencies. The overall equation of the electrochemical reaction is given by,

$$
2 \mathrm{H}_{2}+\mathrm{O}_{2} \Rightarrow 2 \mathrm{H}_{2} \mathrm{O}+\text { Electrical Work }+ \text { Heat }
$$

where hydrogen and oxygen gases are the only reactants and the products are water, electricity and, because this is an exothermal reaction, some heat.

A PEMFC has five main components. A diagram showing the basic PEMFC components is shown below in Figure 1.1. The central element which makes the technology possible is the ion exchange membrane. This membrane facilitates the transport of ionic charge from anode to cathode, which ultimately provides the electrical power for external loads. On either side of the membrane are catalyst layers, which is where the electrochemical reactions occur. On the outside of both catalyst layers are gas diffusion electrodes, which conduct the electrical current and also allow the reactants and products access to/from the catalyst layers. All of these components are assembled together during manufacturing and are collectively referred to as a Membrane Electrode Assembly (MEA). The MEA is placed between two electrically conducting plates, often made of graphite, with gas flow channels engraved into them. These flow channels distribute the reactant gases over the MEA and remove the product water and any unused reactants. In a stack, these plates are also used to circulate cooling water and to provide structural support. 


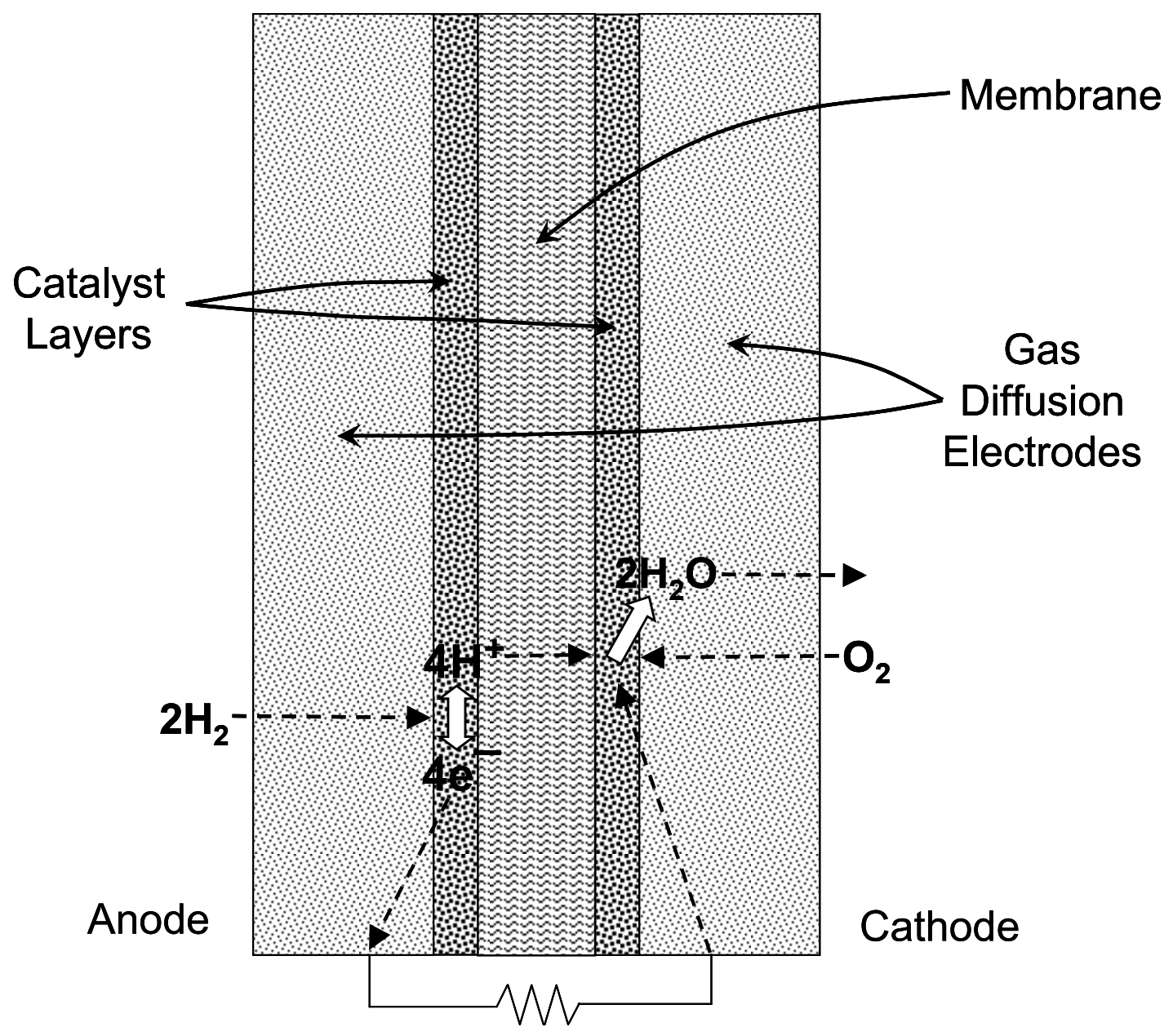

Figure 1.1: Membrane Electrode Assembly

When referring to the fuel cell, the anode is the side of the cell which takes the hydrogen gas and separates the electrons and protons which comprised the hydrogen molecule, and the cathode is the side which combines the protons, electrons, and oxygen atoms to produce water. The half reaction at the anode is,

$$
H_{2} \Rightarrow 2 H^{+}+2 e^{-}
$$

At the anode catalyst layer, the diatomic hydrogen molecule is first split into two atoms of hydrogen which are then ionized, producing two electrons and two hydrogen ions, which are simply protons. The protons are conducted into and through the membrane, which is specifically designed to conduct ions while being electrically insulating. This is how the charge separation is maintained within the cell. The electrons are then conducted through the anode gas diffusion electrode, where, for the case of a single cell, they are collected by the graphite plates and then passed through an electrical load to the 
cathode side of the cell. After diffusing through the membrane to the cathode catalyst layer, the protons are reunited with electrons and combined with oxygen atoms, producing water.

Similarly, on the cathode side of the cell, the oxygen, which usually comes from air, diffuses to the cathode catalyst layer where the diatomic oxygen molecule is separated into two oxygen atoms. Each of these oxygen atoms joins to two protons and two electrons, producing one water molecule. The cathode reaction is given by,

$$
\mathrm{O}_{2}+4 \mathrm{H}^{+}+4 e^{-} \Rightarrow 2 \mathrm{H}_{2} \mathrm{O}
$$

Thus, the overall reaction as given by Equation (1.1) converts two hydrogen molecules and one oxygen molecule into two water molecules while producing an electrical current. Since this reaction involves a separation of the positively charged proton and the negatively charged electron, a voltage is induced across the cell. The reaction, joining hydrogen and oxygen to produce water, theoretically induces a voltage of 1.18 volts at a temperature of $300 \mathrm{~K}$ [6]. A measure of the power output, in Watts, of the PEMFC is simply determined by the electrical power equation,

$$
P_{\text {electrical }}=I V
$$

where, $\mathrm{I}$ is the DC current, in Amps, and V is the measured cell voltage, in Volts. To boost the total PEMFC's power output, cells are connected in a stack to create a higher voltage, just like what is done with batteries. Now, instead of the electrons passing through an external load across every cell, the electrons of Equation 1.2 are passed to the cathode of the neighboring cell. The load, then, is placed across the entire stack.

\subsection{Diagnostic Techniques}

To properly understand the fundamental operation of fuel cells it is critical to use appropriate diagnostics. The "health" of the cell is an important measure, which is not always easily determined simply from the cell voltage. This section will describe the available techniques and justify the use of electrochemical impedance spectroscopy (EIS) as a diagnostic measurement technique for a PEMFC stack. 


\subsubsection{Survey of Diagnostic Techniques}

There have been many methods used to diagnose the operating performance of a PEMFC. Almost always, V-I Polarization Curves, which plot the relationship between voltage and current, are used to illustrate the losses which are predominant at different cell current densities. The voltage is related to the current through Ohm's Law,

$$
V=I R
$$

This is why the cell voltage decreases with increasing current. The total cell resistance is not constant, but is the sum of three loss mechanisms, each of which is dominant for different current ranges. Specifically, these losses are electrochemical activation at low currents, ohmic at mid-range currents and diffusional at the highest currents [7]. The effects these losses have on the actual cell voltage are illustrated in the sample polarization curve in Figure 1.2. An explanation of each loss will follow.

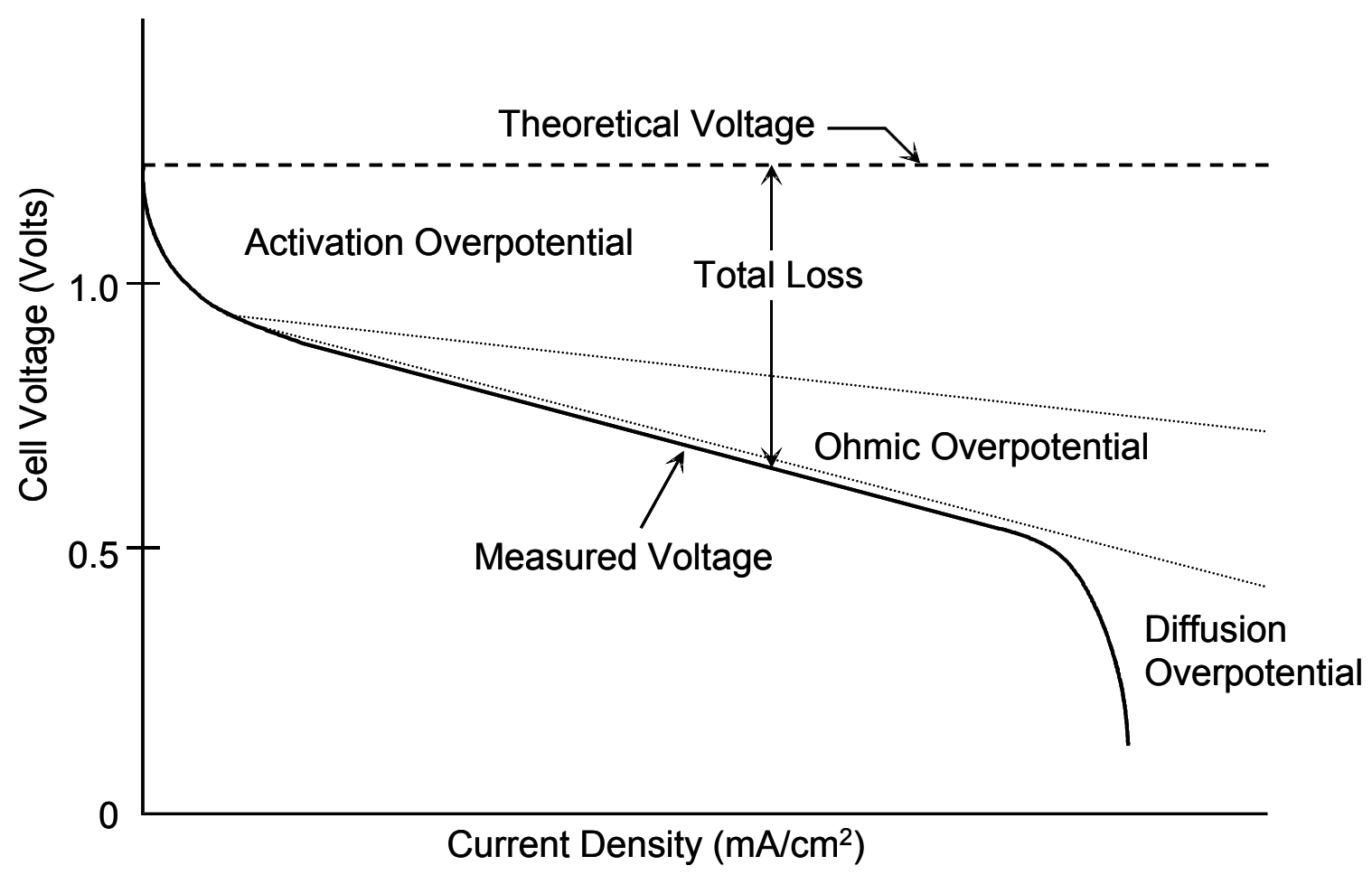

Figure 1.2: Sample VI Polarization Curve

Electrochemical activation losses are due to two electrochemical reactions. The first reaction, in the anode, separates the hydrogen molecule into a pair of electrons and protons. The second reaction, in the cathode, joins together the protons, electrons and 
oxygen atom to form water. Typically, the anode reaction occurs quickly while the cathode reaction is much slower [7]. A slow reaction generally requires more kinetic energy in order to sustain a reaction rate. This is because, the higher the kinetic energy, the more likely the molecules are to react. This kinetic energy can also be thought of as an energy barrier, or threshold, that will inhibit the reaction until that energy level is attained. The amount of energy required to overcome this barrier is called the electrochemical activation energy. So the majority of the electrochemical activation losses are directly attributable to the cathode reaction, and the anode reaction is usually ignored because it has a much lower activation energy. Electrochemical activation losses are the dominant loss at very low currents as the activation energy barrier is overcome.

Ohmic losses are predominant for the middle current range once the effects from the electrochemical activation loss become reduced (the magnitude of the activation loss is proportional to the logarithm of current). The ohmic losses are due to the charged particles (both electrons and ions) passing through electronic and ionic resistive elements in the fuel cell components, including the bi-polar plates, the electrodes, any contact resistance among the interfaces, and ionic resistance in the membrane. Ohmic losses continue to dominate for most of the load current range.

At high currents, diffusional losses start to dominate the nature of the polarization curve. These losses are attributable to diffusion effects from the reactants migrating through the electrodes. Recall, the active reaction sites are on either side of the membrane in the catalyst layers. In order to sustain those reactions, the reactant gases must diffuse through the electrode structure quickly enough to replace the consumed reactants. At high currents, this loss grows exponentially and ultimately limits the maximum current the PEMFC can produce. An air operated PEMFC also must account for the oxygen diffusion through air.

Some other techniques used to provide diagnostics on a PEMFC include current interrupt [8] and physical examination, i.e., through use of a Scanning Electron Microscope [9], etc. Current interrupt consists of allowing the load current on the cell to reach a constant value, then abruptly removing the load while measuring the transient cell voltage response. Immediately after removing the load, the net current will fall to zero and the cell voltage will jump since all ohmic losses are removed nearly instantaneously. 
At this point, the only current in the cell is from the charge stored in the double layer. The electrical double layer is the separation and subsequent build up of charge at the electrode/electrolyte interface. As such, the double layer behaves like a capacitor. The cell's voltage response, then, is the response of the electrochemical reaction returning to equilibrium since there is no longer a path for a net external current. Based on this measured response, RC time constants for the electrochemical reaction can be calculated, where the capacitance $\left(\mathrm{C}_{\mathrm{DL}}\right)$ is from the double layer and the resistance $\left(\mathrm{R}_{\mathrm{CT}}\right)$ is from the charge transfer. Figure 1.3 shows a typical current interrupt response with the inset showing the how the response can be modeled with a circuit.

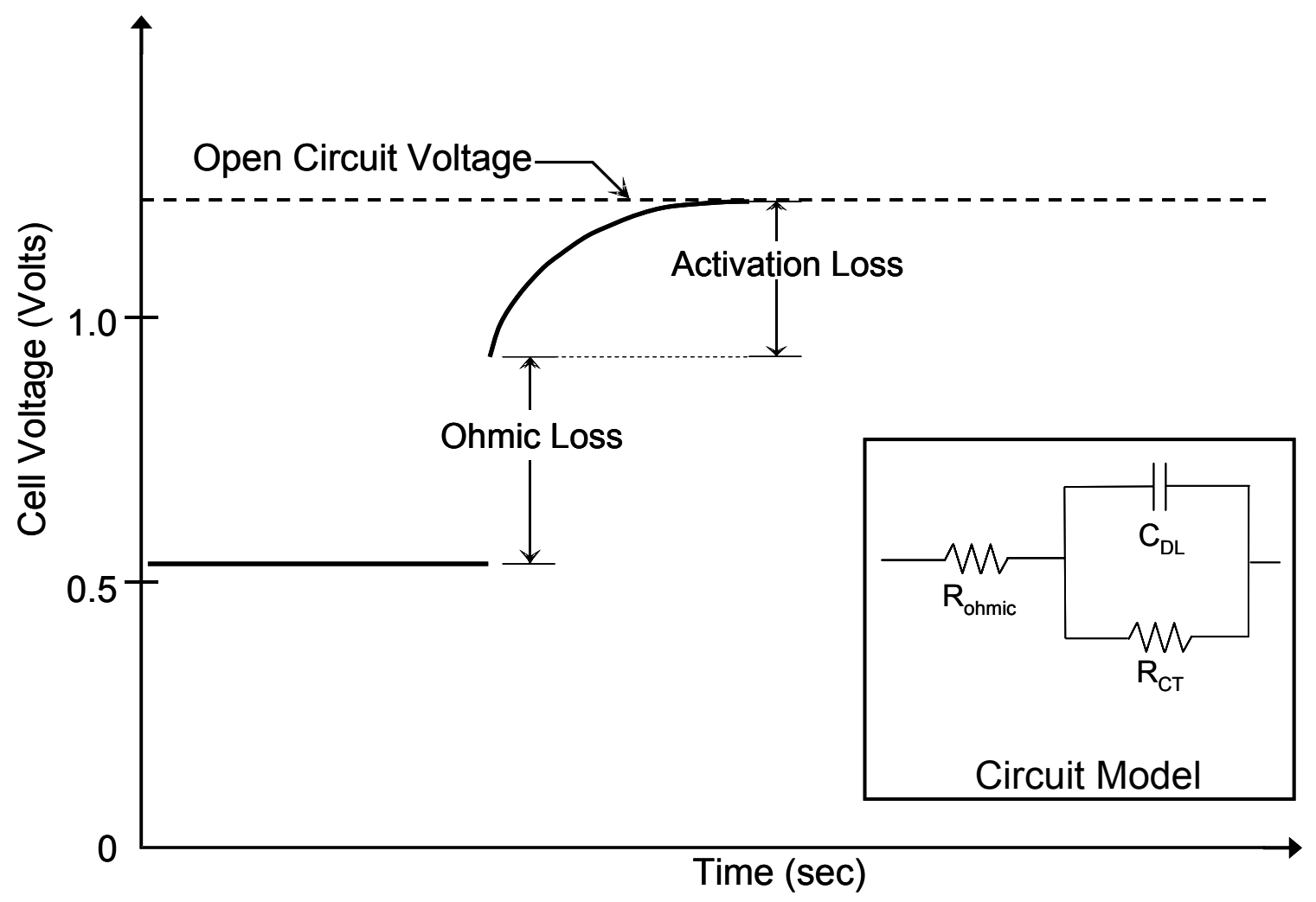

Figure 1.3: Sample CI Curve

Physical examination involves analyzing the structure of the PEMFC's components. This is usually done microscopically using a high powered optical or electron microscope [10]. The desired result is an analysis of the surfaces of the membrane, catalyst layers, and the porous electrodes showing any flaws or maladies introduced from previous operation. This is usually done by making a before and after comparison. 


\subsubsection{Electrochemical Impedance Spectroscopy (EIS)}

The diagnostic technique chosen for this work, in addition to V-I Curves, is electrochemical impedance spectroscopy (EIS). This method has been widely used to examine PEMFC behavior and is sometimes also called AC impedance spectroscopy. To perform EIS measurements on a PEMFC, a small Alternating Current (AC) signal is superimposed onto the Direct Current (DC) of the fuel cell. The impedance response of the cell is then determined for various $\mathrm{AC}$ frequencies by measuring the magnitude and phase components of the total cell current. At each specific frequency, an impedance value for the cell is determined. By performing a frequency sweep of the AC signal, it is possible to generate an impedance response characterizing all the losses that are affecting the fuel cell performance at any given operating condition. This response is usually displayed in the complex plane (also referred to as a Nyquist plot). As an example, the EIS response of the current interrupt circuit model (cf. inset of Figure 1.3) is shown in Figure 1.4. By convention, most complex plane plots are flipped about the real axis, so that the imaginary axis is inverted. This is simply done because most of the responses are capacitive (negative imaginary component).

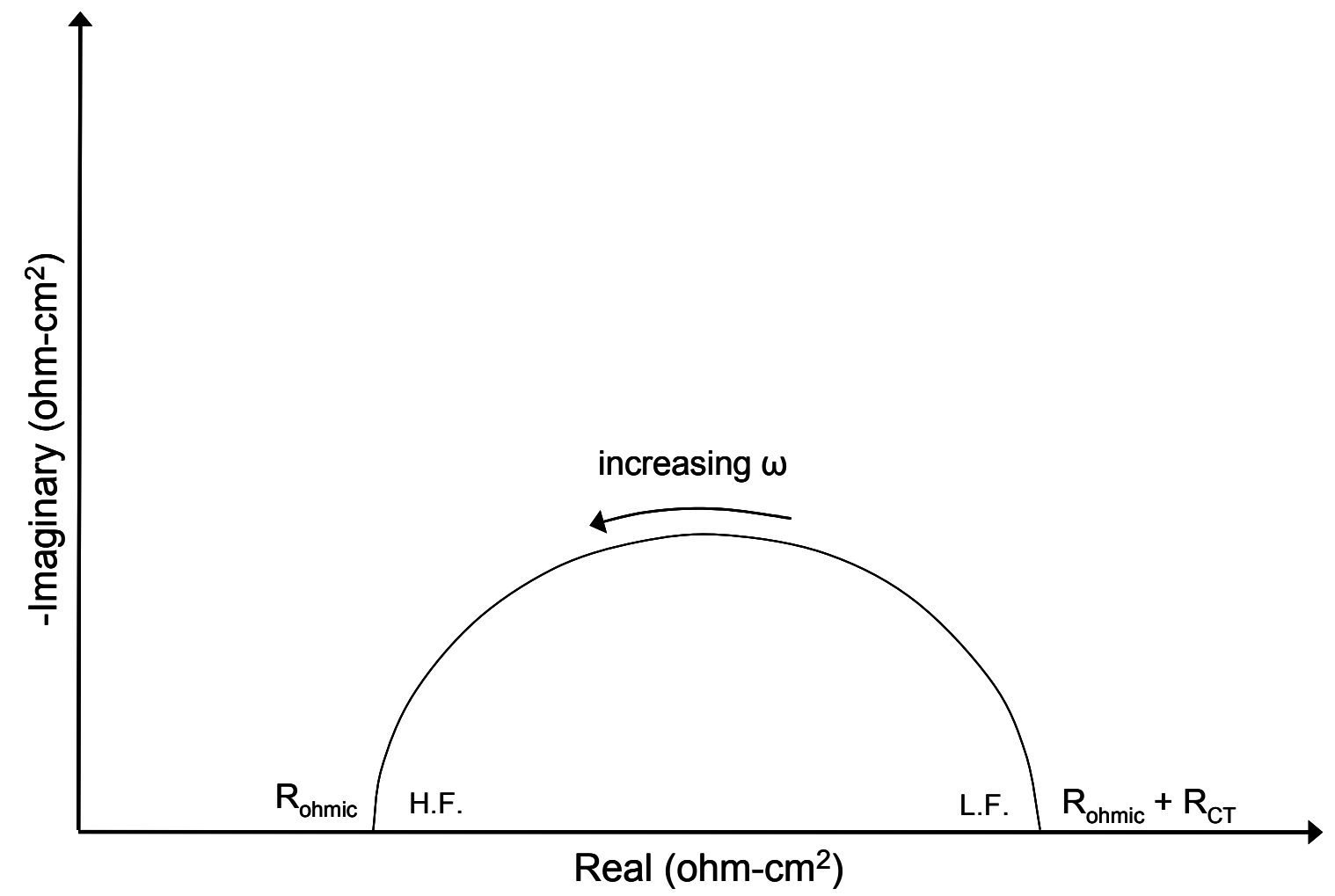

Figure 1.4: Sample EIS Response 
Using EIS has a definite advantage over some of the other techniques listed above. Physical examination, aside from a materials analysis, provides the least amount of performance-related information. This also requires a complete dismantling of the fuel cell stack in order to examine the components and usually destroys the MEA, which is not desirable for online stack diagnostics. Current Interrupt is also somewhat limited in the amount of information provided about the immediate performance of the cell. Often, Current Interrupt has been used to double-check the results determined from EIS. Current Interrupt does provide online diagnostic ability, although the fuel cell can not be gainfully used during the diagnostics test because of the non-continuous current. EIS provides the most performance related information of the three supplements to V-I Curves. It provides online diagnostic ability, during which the fuel cell may be used to provide power, with the stipulation that the load current, and all other parameters, remain constant throughout the measurement. A disadvantage of EIS is the length of time required to perform a measurement. In order to complete a frequency sweep, to capture the long time constants associated with diffusion effects, frequencies on the order of 10 $\mathrm{mHz}$ must be used. Therefore, to simply obtain one cycle at that frequency, which is the minimum requirement, the measurement time for that frequency alone could be no shorter than $100 \mathrm{sec}$. This is found by calculating the period of the signal at the frequency of $1 \mathrm{mHz}$

$$
T[\mathrm{sec}]=\frac{1}{f[H z]}
$$

\subsubsection{Interpretation of EIS Measurements}

An additional difficulty often cited when using EIS is interpretation of the results. Since EIS only measures the response of the fuel cell, there is no direct phenomenological measurement being performed [11]. Therefore, since only indirect responses are measured, it is up to the researcher to interpret the EIS measurements and assign associated physical phenomena to the measured responses. This is not a trivial task, and as will be discussed in the following section, there is some disagreement within the research community about the interpretation of the measured responses. To go back 
to the example response of Figure 1.4, as has already been demonstrated, an equivalent circuit model could be developed to match the response. To generalize, the circuit shown in Figure 1.5 would generate a similar response.

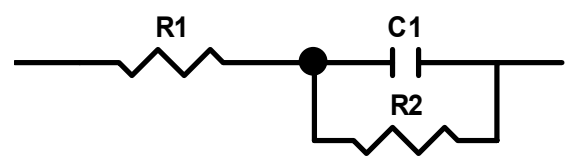

Figure 1.5: Equivalent Circuit Diagram

The value of resistor R1 would relate to the resistance at the high frequency intercept of the real axis. The values of capacitor $\mathrm{C} 1$ and resistor $\mathrm{R} 2$ relate to the semicircle shape. $\mathrm{R} 2$ is the resistance from the high frequency intercept on the real axis to the low frequency intercept. The value for $\mathrm{C} 1$ is based on the frequency range that generated the semicircle. It can be calculated by solving Equation 1.7

$$
\omega_{\text {max }}=\frac{1}{(R 2)(C 1)}
$$

$\omega_{\max }$ is the frequency (in $\mathrm{rad} / \mathrm{sec}$ ) at the top of the semicircle.

\subsection{Related Work}

The extent of PEMFC research to date has largely been centered on characterizing the properties of single cells. There has been a lot of work published examining various parameters and physical features associated with a PEMFC. Some examples include the characteristics of the different proton-conducting membranes used $[12,13]$ including the chemical composition, conductivities, water retention, and membrane thickness. Other parameters include catalyst loading and utilization within the Gas Diffusion Layer $[14,15]$, humidity of the fuel and oxidant gases, ambient temperatures, and bipolar plate flow field design. Work has also been done analyzing the design and manufacturing process of the elements of a cell [16].

It is also recognized that individual cells will not be able to supply the necessary power and/or voltage requirements of typical applications. In order to mitigate this, fuel cell stacks need to be used. Fuel cell stacks combine individual cells in series to boost 
the voltage output. Stacks can consist of only a few cells or can number into the hundreds or even thousands of cells. Research has been conducted on some of the parameters associated with a stack configuration. The physical arrangement of the cells and their connections has been examined by Jiang [17]. Chu [18,19] and Qi [20] have examined the effects of ambient temperature and gas humidity in a stack arrangement. However, there has not been much consideration given to the conditions that may arise during operation.

The remainder of this section will cover in more detail some of the fuel cell and EIS work that has been conducted. First, single cell EIS work will be summarized to show how the technique has been used. Then, a brief discussion of fuel cell models will be presented. Finally, the work describing fuel cell stack development and the resulting complications will be discussed.

\subsubsection{Single Cell EIS Diagnostic Work}

EIS is a well-known tool for characterization of various electrochemical systems [21-24]. The most commonly studied systems are corrosion and coatings, batteries, and fuel cells. In the early 1990's, EIS was first used as a diagnostic technique for fuel cells. Parthasarathy, et al. [7] looked at the interface between the platinum catalyst and the Nafion membrane on the cathode side of a cell running on hydrogen and oxygen. Parthasarathy, et al. tried to quantize the kinetic parameters associated with the Oxygen Reduction Reaction. The cathode was the focus of their research, because, of the two electrode reactions (Hydrogen Oxidation Reaction (HOR) at the anode and Oxygen Reduction Reaction (ORR) at the cathode), the ORR is a much slower reaction. It was believed that since it was the slower reaction, the ORR's effects would dominate and overshadow the effects of the HOR. The diffusion of oxygen through the Nafion and other parameters of the membrane are also reported. The work of Parthasarathy, et al. provides much of the foundational work for EIS measurements and interpretation. The authors also attempted to derive an equivalent circuit to describe the impedance behavior. They were unable to develop a single circuit that would apply over all current loadings, and instead had to break the EIS data into two regions, categorized by cell potential, shown in Figures 1.6 and 1.7. 


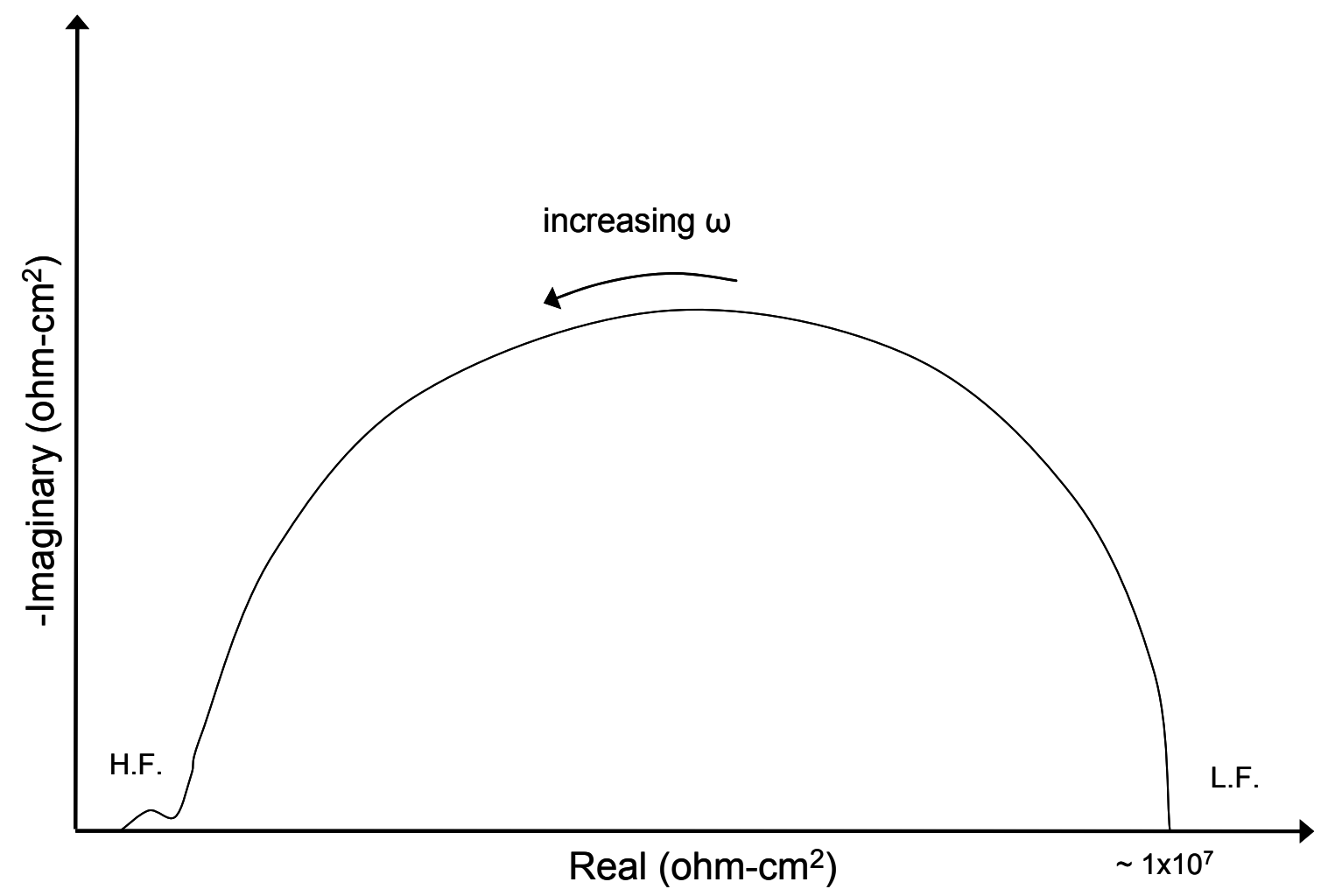

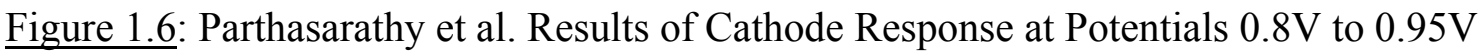

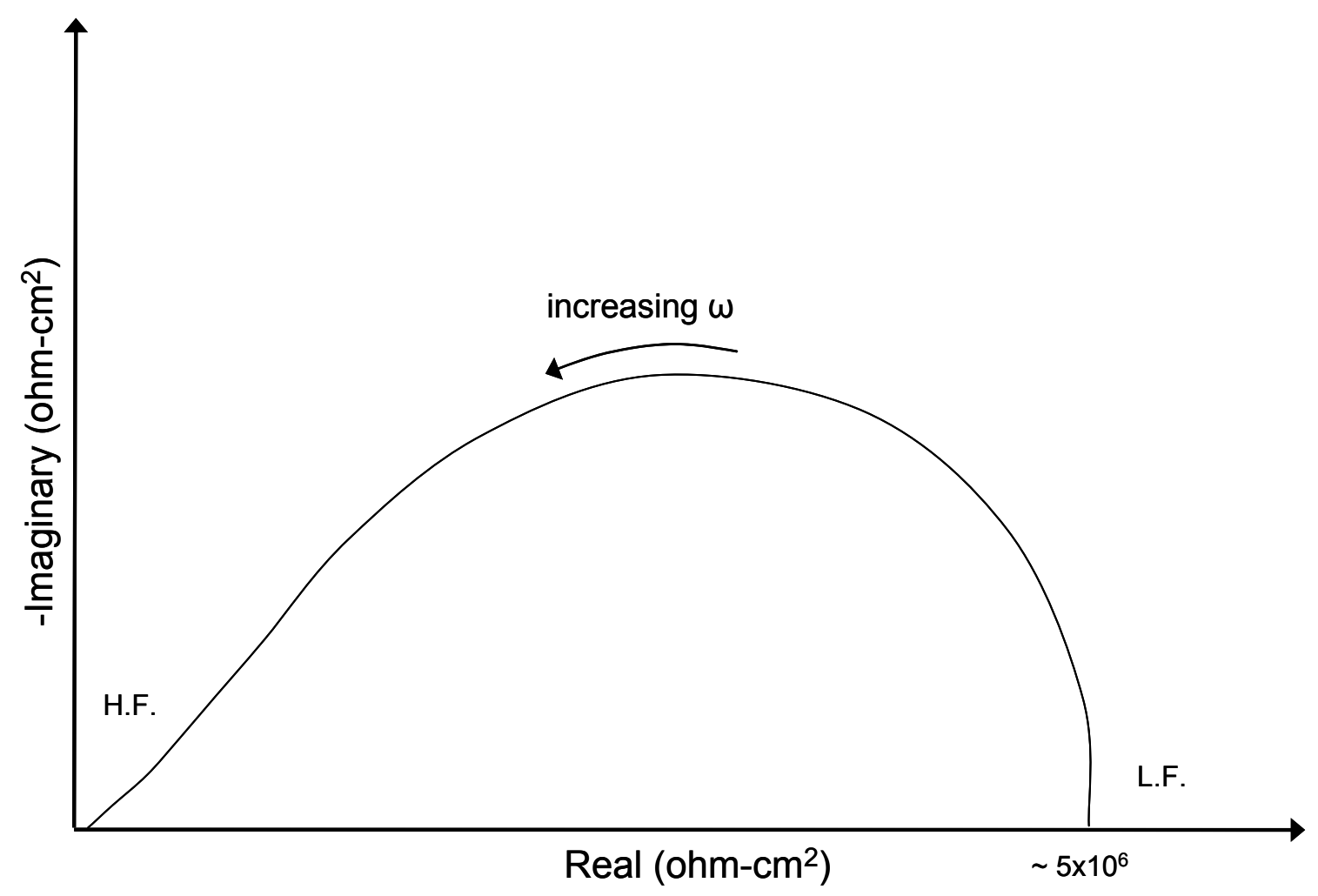

Figure 1.7: Parthasarathy et al. Results of Cathode Response at Potentials $0.35 \mathrm{~V}$ to $0.75 \mathrm{~V}$ 
Extending this work, Springer, et al., [25] also worked with the cathode side of the cell while taking EIS data and worked on developing a model. Springer, et al. worked with both oxygen and air fed cathodes. They compared the experimental data to the model data and showed good agreement between the two. The authors also offered explanations for the processes of each of the shapes present in the EIS data. Parthasarathy, et al. [7] was more concerned with calculating parameter values, probably because they were trying to fill in values for an equivalent circuit. Springer, et al., was better able to relate the data to the physical processes that were occurring and offers a more thorough description of the EIS data. For the oxygen fed case, the authors identify one loop, not necessarily semicircular, on the complex plot of the impedance data. They attributed the EIS response shapes to the following: the high frequency section is due to the charge transfer associated with the ORR, and when air is used as the oxidant gas there is another low frequency feature due to oxygen diffusion through the backing layer, as shown in Figure 1.8.

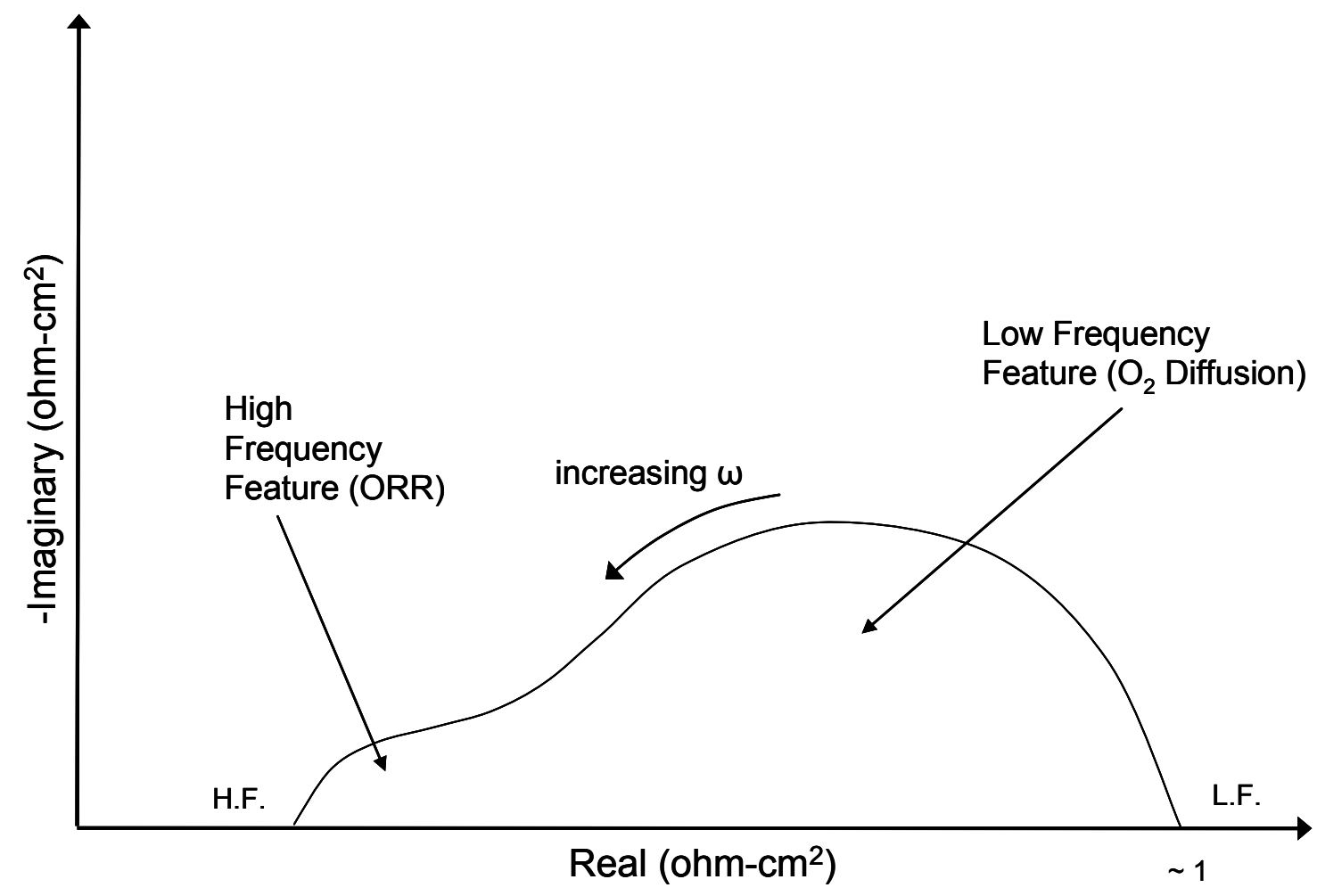

Figure 1.8: Springer et al. Results for Air Cathode

In addition, Paganin, et al. [26] suggests another low frequency loop is present in the EIS data at frequencies lower than were previously investigated by Springer, et al. 
The authors state that when pure Oxygen is used as the oxidant gas and when the cell potential is low (high overpotential), there is a low frequency loop they attribute to product water diffusion through the membrane. They argue when pure Oxygen is used, the cathode always has enough Oxygen to sustain the ORR. Therefore, the authors attribute the low frequency loss mechanism to the dehydration of the membrane at the anode side, since a dry membrane will not conduct ions effectively.

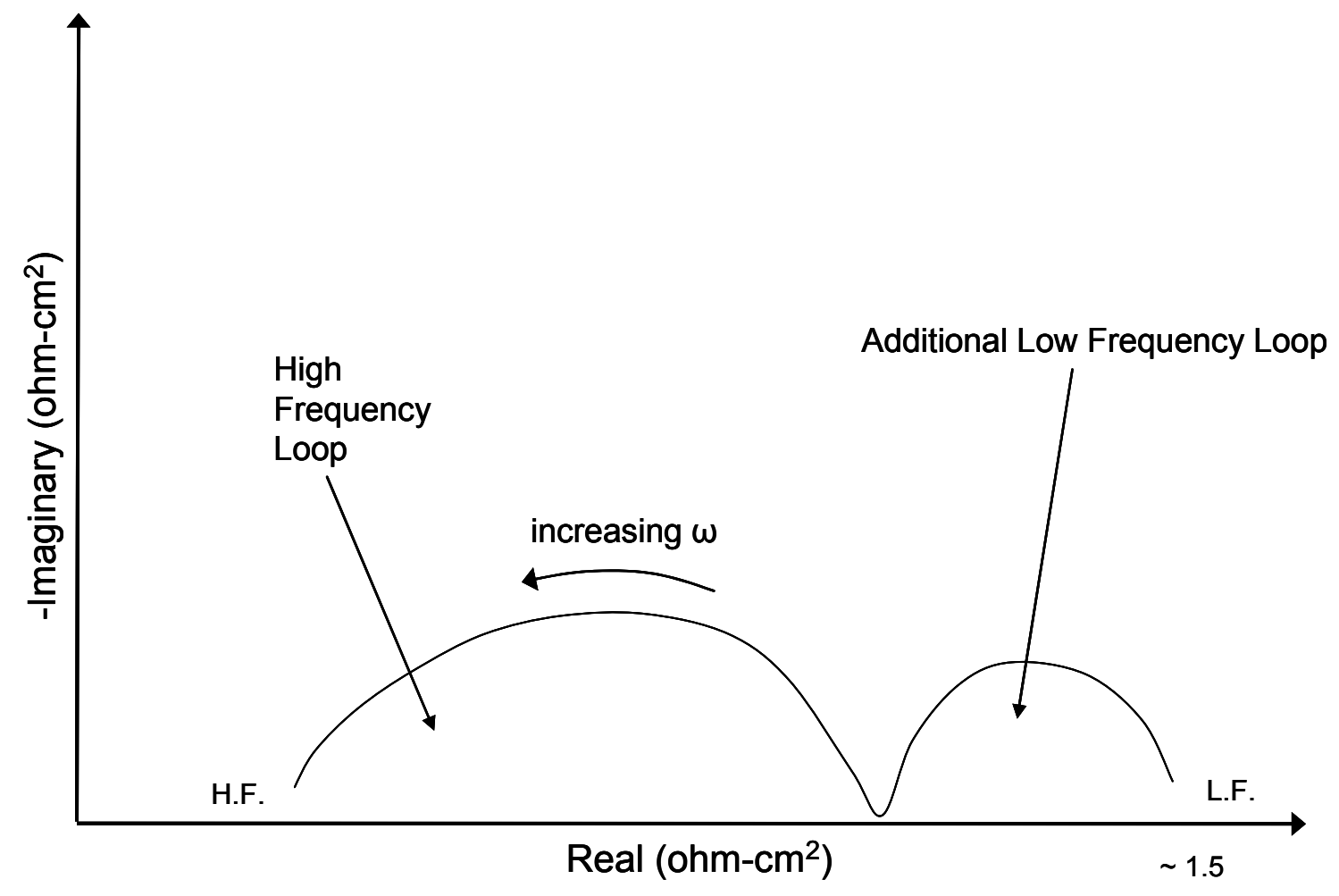

Figure 1.9: Paganin et al. Results for Oxygen Cathode

The authors also confirm that when air is used, only one loop is present (at higher frequencies). The authors explained that the oxygen diffusion then becomes more of a limiting condition than the membrane hydration (because less oxygen is present). They do, however, suggest that there is a possibility for pressurized air systems being limited by the water diffusion as well (which would add the low frequency loop to the EIS curve).

There are other explanations and interpretations given for the EIS response of a PEMFC, especially the low frequency information. Freire, et al. [27] suggests flooding in the cathode and membrane hydration are both contributing factors. Ciureanu, et al. [29] summarizes this difference of opinion regarding the EIS responses of a PEMFC and 
identifies three interpretations of the low frequency loop, as given by Springer, et al. [25], Paganin, et al. [26], and Wagner, et al. [28].

This brings up an interesting point in the literature. There is some uncertainty regarding the origins of the low frequency loop in the EIS response of a PEMFC. A commonly accepted model of a fuel cell electrode, the flooded-agglomerate model, as described in Raistrick [30], states there should be three impedance loops, a high frequency loop from the double-layer capacitance, an intermediate loop due to "agglomerate dynamics", and a low frequency loop from the thin film diffusion. Ciureanu, et al. [29] suggests that the reason no more than two impedance loops have been reported experimentally is that at least two of the processes time scales overlap and are not distinguishable in the EIS data.

This discussion illustrates some of the difficulties in assigning physical phenomena to EIS responses. The interpretation of the data collected is not straight forward and is, to some degree, subjective. As has already been described, some features in the EIS data can overlap other features, making the two indistinguishable. Orazem [11] addresses this issue extensively as it applies to the interpretation of EIS data for use in a model or equivalent circuit. Despite some of these difficulties, EIS is useful when identifying various overpotentials which affect PEMFC performance.

To generalize about the EIS response of an air operated cell, most work $[25,26,27]$ presents a similar trend as the cell current is increased from initially open circuit conditions, even though there may be different interpretations given. The trend that is evident in the literature is for the high frequency intercept of the real axis to remain relatively constant, while the low frequency intercept usually starts at a high value, quickly approaches a minimum value, then slowly increases as the cell current continues to increase. This is summarized in Figure 1.10. 


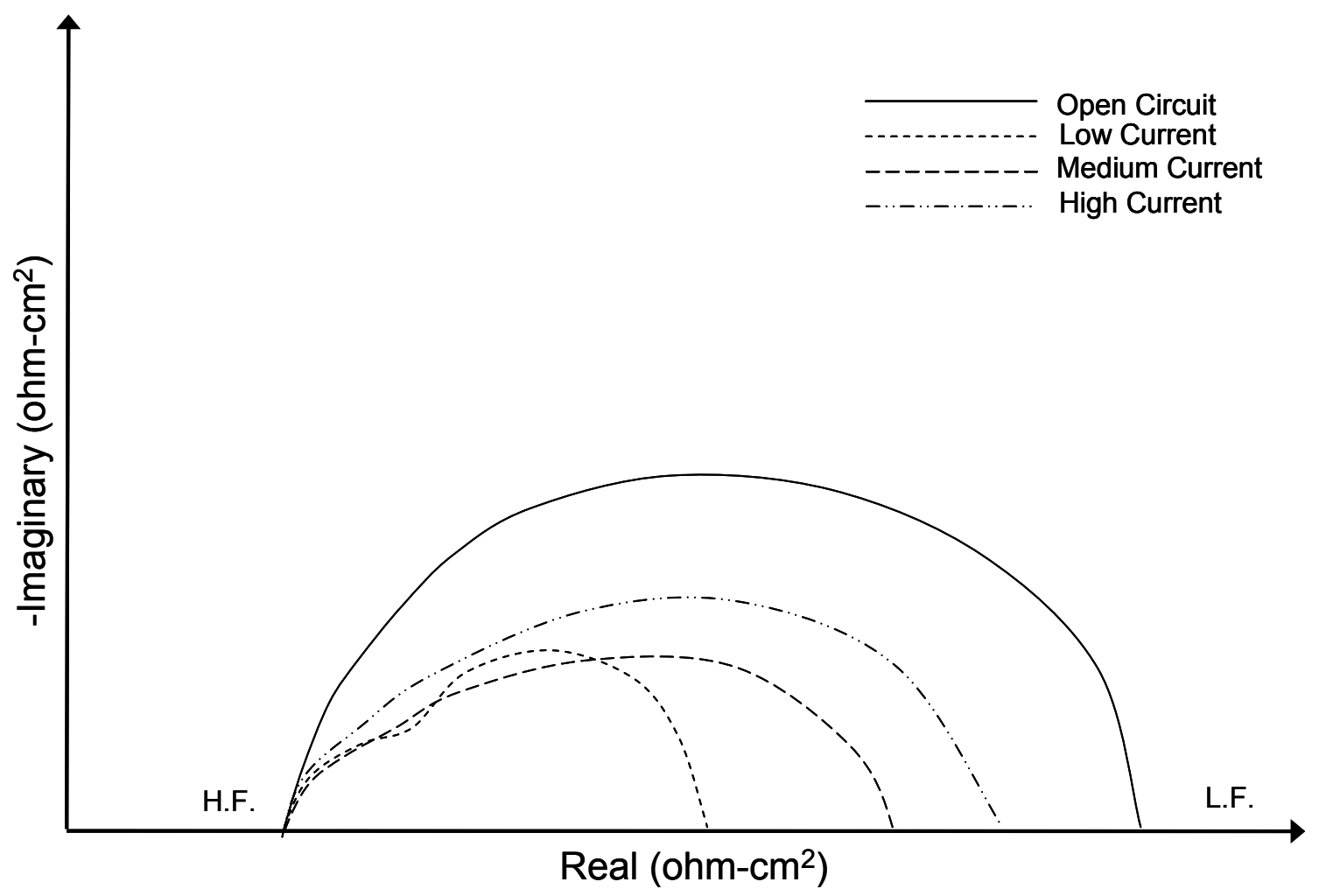

Figure 1.10: General Trend of EIS Response with Current

In order to try to remove the guesswork from EIS data interpretation, reference electrodes have been employed. A reference electrode, as used by Diard [32], allows for the separation of the anodic and cathodic effects. Thus, the addition of a reference electrode helps identify the source of the overpotentials. However, not all PEMFC setups are conducive to using reference electrodes, mostly due to the challenges of making a physical connection to a third electrode. Therefore, the most common method of clarifying EIS data is to categorize the response by varying specific experimental parameters (i.e. cathode air flow rates) until certain features (i.e. a low frequency loop) become evident in the EIS data. From this, relationships can be developed and entered into a model. Then the model can be used to replicate experimental data and predict the overall behavior of the fuel cell. If the model fits the experimental data for all parameters' values, then it is generally considered to be a good model that accounts for the physical processes that drive the electrochemical reaction.

In summary, a large amount of work has been done investigating a single cell PEMFC using EIS. Most of the focus has been directed at cathode improvements, with emphasis on enhancing the ORR and facilitating $\mathrm{O}_{2}$ transport to the catalyst layer. 
Hydration levels of both the gases and the membrane have also been studied using EIS. Although there are some challenges and uncertainties when applying EIS to PEMFCs, EIS has been able to provide useful insights into the operation of single cell PEMFCs.

\subsubsection{PEMFC Models}

There has been a great deal of improvement in the accuracy of the PEMFC models within the last five years. Much of the early modeling work done by Springer [33,34] and Bernardi [35] established a solid modeling foundation. These models assumed basic stable operations of the fuel cell and simply tried to describe the losses that occurred and match the model prediction to the experimental V-I curve. This work was extended by others and progressed into a more detailed examination by including two dimensions to better understand heat and water management and diffusional limitations by Singh [36] and three dimensions by Berning [37]. There also has been work done to account for non-uniform parameters like temperature and water distributions by Maggio [38] and You [39]. Lee [40,41] has looked at MEA properties and the effect of a stack arrangement on fuel cell operation.

\subsubsection{PEMFC Stacks}

There has also been much work done in developing PEMFC technology itself, especially in the last decade. However, there is still much more research that needs to be done dealing with PEMFC stacks. Right now, the technology is at a very important stage, as the fundamental research is being shifted into applied research. There are still many problems associated with fuel cell stacks that have yet to be resolved. Jiang and Chu $[18,19,42,43]$ have done a great deal of work identifying some of the issues involved with operating fuel cell stacks. They specifically identify mass transfer of water and oxygen, temperature gradients, and cell humidification as some of the major challenges

that are hindering the implementation of this technology. Ahn [9] and Mennola [8] have also reported variations among cell resistance and temperature values at different points in the stack. 


\subsection{Statement of Work}

At present, it is not well understood how cells are influenced by conditions within a stack. The behavior of an individual cell is well understood from the use of diagnostic techniques such as EIS and detailed models have replicated the cell's behavior. The operating conditions inside a stack are not the same as an isolated cell. An ideal stack would behave like a scalar multiple of a single cell. However, due to the conditions described above, and also including cell-to-cell variability, along with differences in flow rates, cells within a stack can be operating under different conditions. There is the need to combine the technique of EIS, which is, arguably, the single most informative diagnostic technique for a single cell, with fuel cell stacks to identify and investigate some of the issues that are inherently present in stack operation. A fuel cell stack is only as healthy as the weakest cell. Therefore it is imperative to keep all cells operating at an optimum level, which, at present, requires more detailed knowledge about stack operation.

This work will investigate the possible extension of EIS as a diagnostic technique for PEMFC stack optimization. This thesis will demonstrate the ability to make EIS measurements on a small, four cell PEMFC stack. The experimental results will be compared to simulations from a model of the stack.

\subsection{Outline of Thesis}

The remainder of this thesis is divided into four chapters. The next chapter will describe the experimental aspects of this work including the setup that was used, the test cases, and present the data that was collected from the stack. Chapter Three deals with the model development and presents the results of the model simulations. Chapter Four is a discussion and comparison of the results of the experimental and modeling work. Finally, Chapter Five provides concluding remarks as well as suggestions for future work in applying EIS to PEMFC stacks. 


\section{Chapter 2: Experimental Work}

Impedance response measurements were performed on a four cell PEM fuel cell

stack. The measurements were made using Solartron Analytical impedance equipment, which was controlled via laptop PC using ZPlot/ZView software from Scribner Associates, Inc. The following sections describe the equipment used, the connections that were made in order to perform impedance measurements, the software setup, and the procedures followed to perform the impedance response measurements.

\subsection{Experimental Setup}

This section describes the physical setup of the experiment, including the equipment used during this work and how the connections were made.

\subsubsection{PEM Fuel Cell}

The fuel cell was a custom designed four cell Proton Exchange Membrane stack using hydrogen gas $\left(\mathrm{H}_{2}\right)$ as the fuel and air as the oxidant. Each cell consisted of a Membrane Electrode Assembly (MEA) and two graphite plates, one on either side of the MEA. The graphite plates were each $0.3175 \mathrm{~cm}$ in thickness and had serpentine channels engraved into the side adjacent to the MEA to allow fuel and air to pass over the MEA. The custom MEAs were ordered from Lynntech Industries Ltd. and used Nafion ${ }^{\circledR} 112$, manufactured by DuPont ${ }^{\mathrm{TM}}$, as the membrane material. The active area of the MEA, the area with the catalyst layer and gas diffusion electrodes, was approximately $134 \mathrm{~cm}^{2}$. The anode catalyst layer was $20 \% \mathrm{Pt}$ on carbon with $45 \mathrm{wt} \%$ Nafion impregnation and had a $0.4 \mathrm{mg} / \mathrm{cm}^{2}$ platinum loading. This means that the catalyst layer consisted of $20 \%$ platinum and the other $80 \%$ was a carbon particle support. The carbon allows less platinum to be used by allowing the platinum particles to be smaller and more evenly dispersed. This provides a high utilization of the platinum catalyst. The platinum loading tells how much platinum is distributed onto a unit area. The Nafion impregnation provides a better union between the catalyst layer, where the ions originate, and the membrane, which transports the ions to the cathode. The cathode catalyst layer consisted 
of pure Pt Black with $8 \mathrm{wt} \%$ Nafion impregnation and had a platinum loading of $4.0 \mathrm{mg} / \mathrm{cm}^{2}$.

The fuel cell stack was formed by electrically connecting the four cells together in series. On either side of each cell a separator plate was inserted to allow for easy access for instrumentation purposes. These separator plates were made out of stainless steel and were $1.905 \mathrm{~cm}$ thick. On the top and bottom of the stack, gold-plated copper end plates served as current collectors. Teflon ${ }^{\circledR}$ gaskets were placed between all stack elements to ensure there were no leaks. Each cell had a valve on the fuel input so the fuel flows to each cell could be controlled independently as is fully described in Hensel, et al. [44]. A common manifold distributed air to each of the cells. An Avtron resistive load bank was used to sink the DC current generated by the stack.

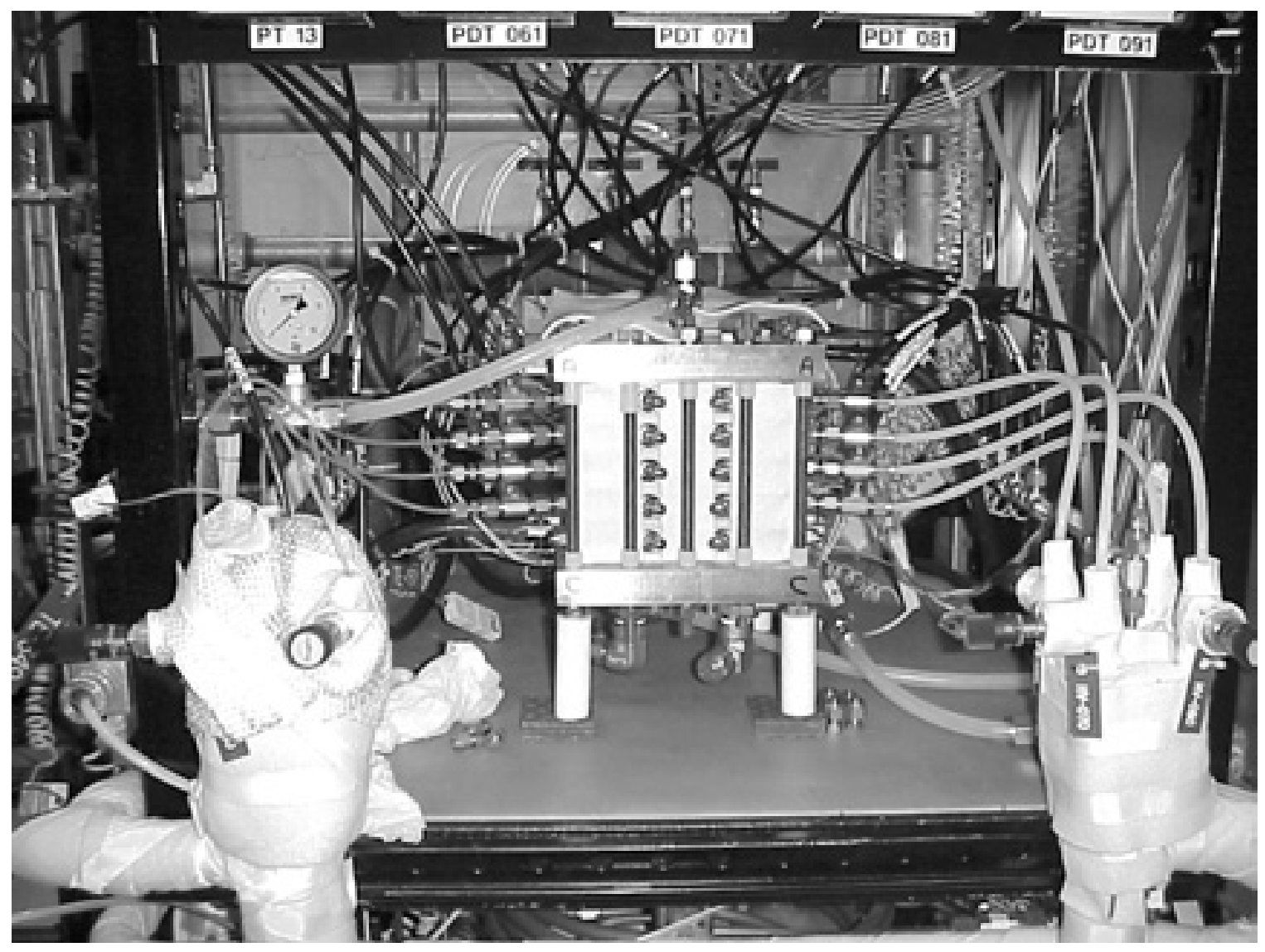

Figure 2.1: Image of PEMFC Stack 


\subsubsection{Impedance Equipment}

The impedance equipment used for the measurements were the Solartron 1260 Frequency Response Analyzer (FRA) and the Solartron 1287 Electrochemical Interface (ECI). The 1260 FRA is the instrument that does most of the work when measuring an impedance response, and can be used in a standalone configuration. The 1260 is used to generate the $\mathrm{AC}$ signal that provides the small signal perturbation. The FRA also measures the response on the cell due to the $\mathrm{AC}$ signal by measuring the magnitude and the phase of the voltage on either side of the cell. The 1287 ECI extends the operational specifications of the $1260 \mathrm{FRA}$ so that the measurement can be performed on a wider array of electrochemical devices. The 1260 and 1287 were connected together as described in the Solartron documentation. To make the connection there are three signals that are passed between the equipment. The 1260 must transfer the $\mathrm{AC}$ signal to the 1287 in order to apply it to the fuel cell. In return, the 1287 relays back the current and voltage measurements to the 1260 to compute the impedance response. This is diagramed in Figure 2.2.

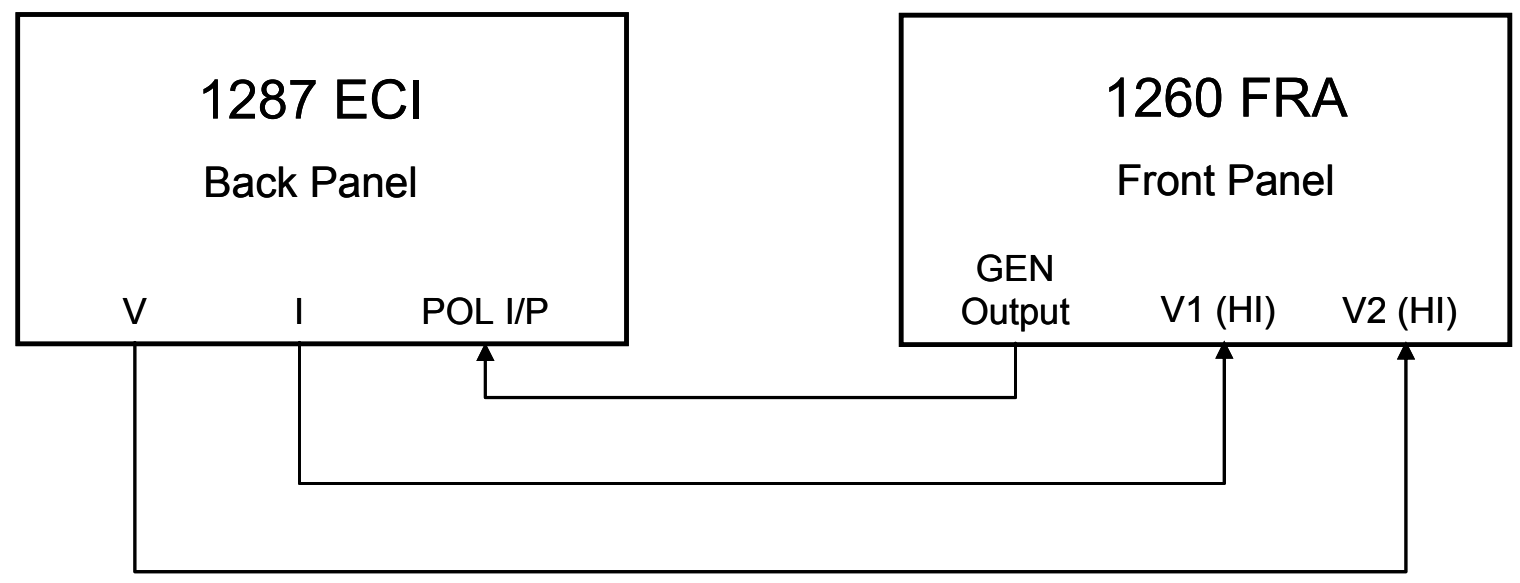

Figure 2.2: 1260/1287 Connections

The 1287 has four leads, each of which is labeled according to how it is connected to the electrochemical cell under test. The leads consist of the Working Electrode (WE), the Counter Electrode (CE), Reference Electrode 1 (RE1) and Reference Electrode 2 (RE2). All four leads are accessible from the front panel on the 1287 via a BNC terminal. When connecting the leads to the fuel cell, only the center pin of the BNC is used, leaving the outer connection of the BNC electrically isolated. This is because the 
impedance equipment has the ability to make voltage measurements when the reference voltage is not on a common ground, in which case the outer BNC connections are used. This functionality is not required for making fuel cell measurements but is still the required setup.

Once connected to the fuel cell, the WE and CE are the two electrodes the AC signal propagates between. The $\mathrm{CE}$ lead transmits the AC signal into the fuel cell, while the WE lead measures the total current through the stack. The 1287 takes this current measurement and exports the signal to the 1260. The other two leads, RE1 and RE2, are used to measure the voltage difference across the stack. This voltage signal is likewise sent to the 1260 and is used to determine the impedance response of the stack.

The Solartron equipment can measure an impedance response in one of two ways. The two techniques are referred to as Constant Voltage (CV) and Constant Current (CC). The CV technique allows the user to specify the nominal potential bias across the cell. As an example, to find the impedance response when the fuel cell has a total overpotential of $200 \mathrm{mV}$, that value can be entered as the constant voltage bias value, and the 1287 will sink enough current until the cell potential is $200 \mathrm{mV}$ less than the Open Circuit Potential (OCP). Once the cell is at that operating condition, the impedance response will be measured. The $\mathrm{CC}$ technique is similar, except it directly specifies the DC current bias.

\subsubsection{Load Bank}

The load bank used is a resistive load bank manufactured by Avtron Manufacturing, Inc. The load bank is model number K492 and is calibrated for 3VDC operation up to 120A. The load is engaged by applying a combination of resistors connected in parallel. There are a total of ten resistors ranging in values from $3 \Omega \pm 1 \%$, corresponding to a $1 \mathrm{~A}$ load, down to $0.15 \Omega \pm 1 \%$, corresponding to a $20 \mathrm{~A}$ load, each of which can be independently applied by a toggle switch. The load bank is capable of applying any DC current load from as small as $1 \mathrm{~A}$ up to the full scale 120A, in increments of $1 \mathrm{~A}$.

Since the load bank is passive, the available resistance values are fixed. The model K492 is designed to operate on a 3V power supply, so all the resistors are calibrated to give the rated DC current if and only if the power supply is at $3 \mathrm{~V}$. If the 
power supply is over $3 \mathrm{~V}$, the load bank will draw current greater than the rated value, and if the power supply is less than $3 \mathrm{~V}$, the load bank will not draw the rated current value. This effect must be considered when connecting the fuel cell to the load since the fuel cell does not operate at a fixed voltage, due to its dependence on current.

\subsection{Software}

This section describes the software that was used to control the Solartron equipment via a laptop PC. This includes a discussion of how to set up the software before the experiment and how to use the software to make an impedance response measurement. This section will also describe how to analyze the data and model an equivalent circuit. For details not discussed in this section, or for a more thorough description, the reader is referred to the operating manuals published by Scribner Associates, Inc. $[45,46]$.

\subsubsection{User Interface of Software}

The software used for collecting and displaying the EIS data is licensed by Scribner Associates, Inc. There are two programs that came in the software bundle. The first program, ZPlot, is used to setup and control the impedance measuring equipment, which, for this work, was the Solartron 1260 FRA and Solartron 1287 ECI. The other program, ZView, is used to display the data and model equivalent circuits. The software version used during this work was version 2.2 for both ZPlot and ZView. The User Interface for ZPlot is shown in Figure 2.3. This is the main window from which all aspects of the experiment can be controlled, including the type of measurement as well as any setup related parameters. 


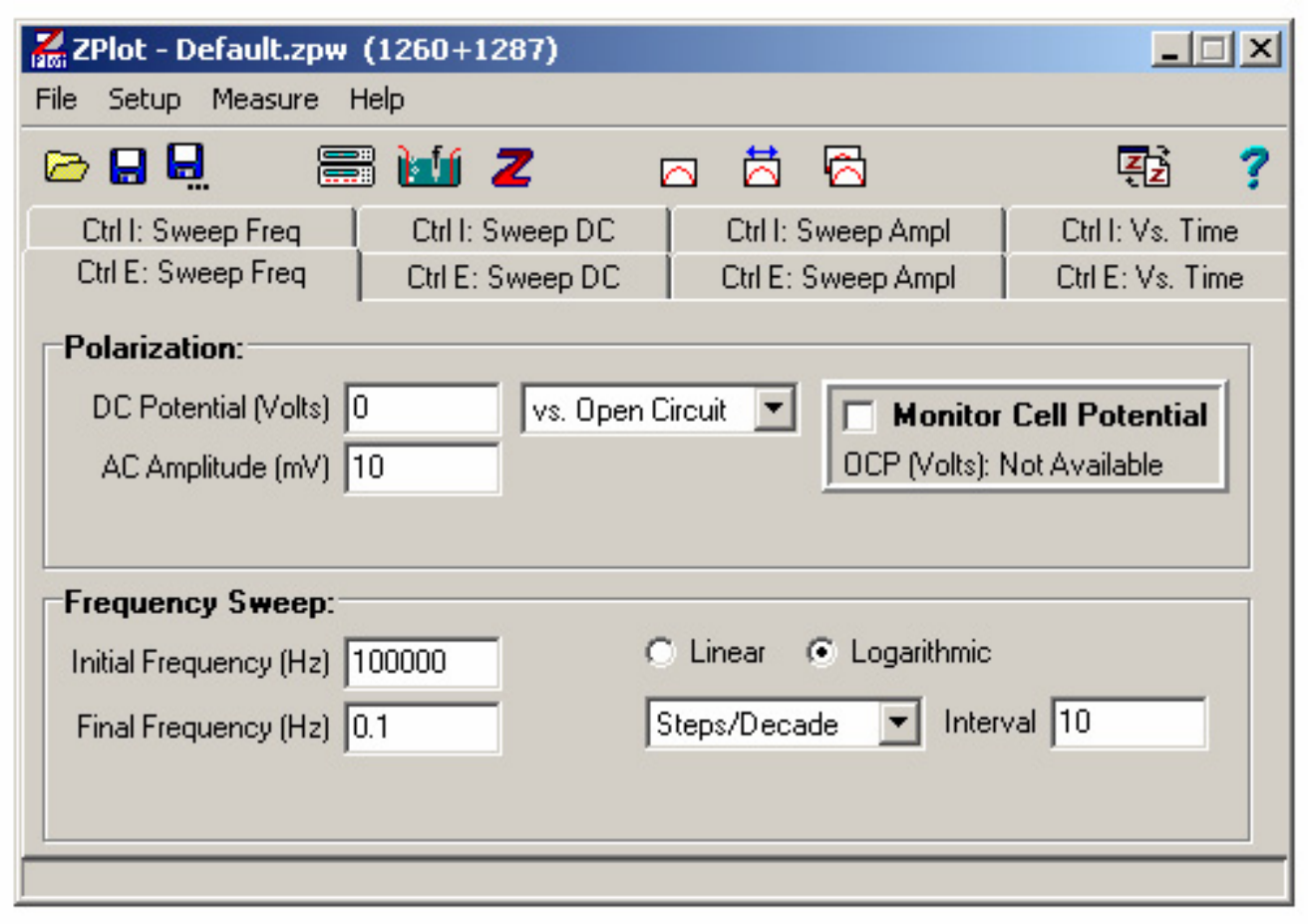

Figure 2.3: ZPlot User Interface

Once an impedance response measurement has been started, the ZView software can be used to display the data in real time along with data that has already been collected. ZView can also be used to estimate an equivalent circuit model or allow the user to derive an equivalent circuit and show the corresponding impedance response. The main window of ZView is shown in Figure 2.4. 


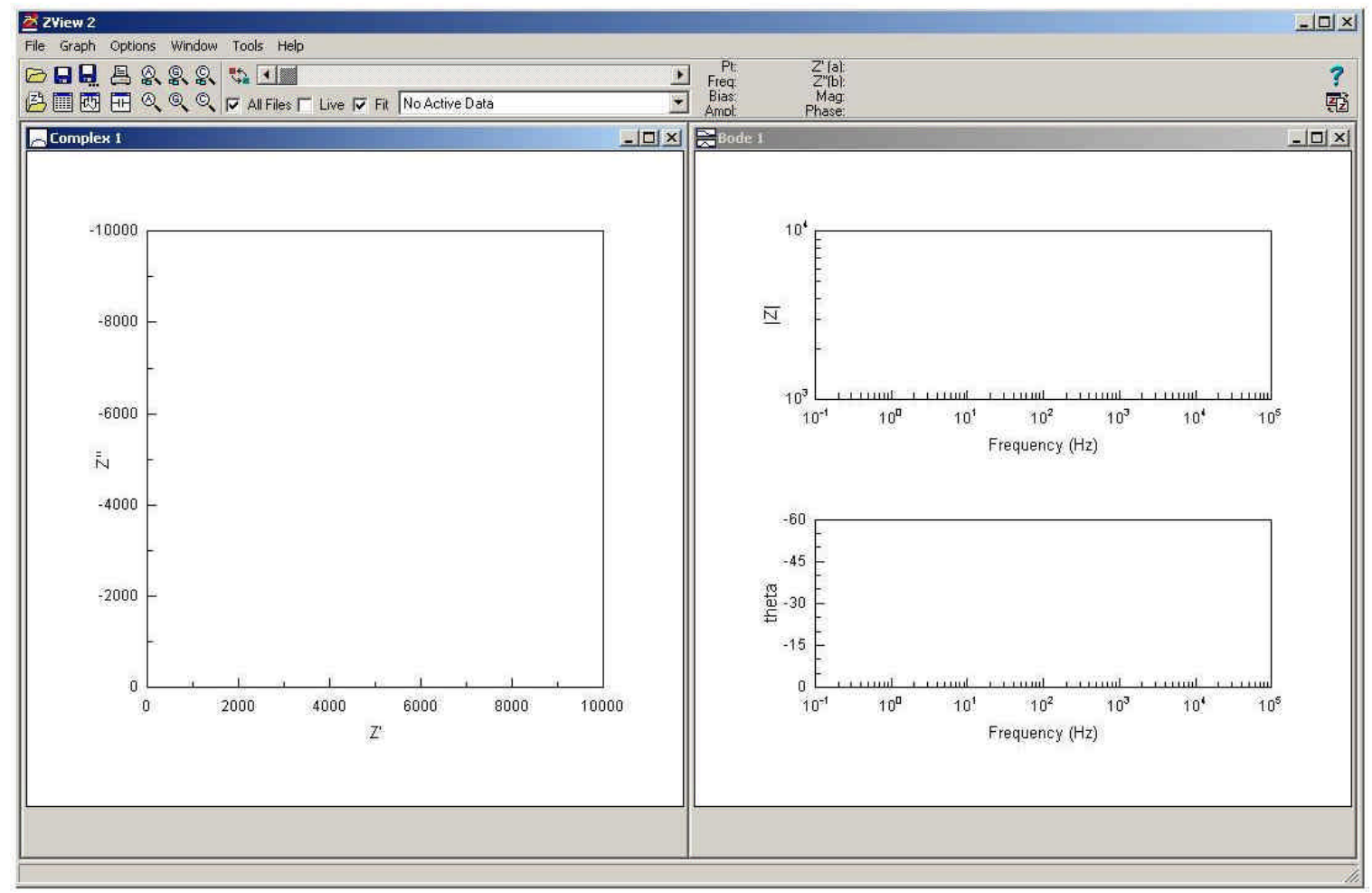

Figure 2.4: ZView User Interface

\subsubsection{Overview of ZPlot}

ZPlot is quite flexible and can accommodate several different manufacturers' equipment. Before any measurements can be made, ZPlot must be setup to control the equipment in the desired configuration. This is done through the 'Setup' menu on the menu bar. First, the equipment being used in the experiment needs to be selected by choosing the 'ZPlot' item of the 'Setup' menu. Once hat has been entered, ZPlot provides full control over the instrument via a GPIB connection. To configure the equipment for the experiment, select the 'Instruments' item, also on the 'Setup' menu. In the case of the 1260/1287 setup, there will be three abs to configure - Potentiostat, Galvanostat, and Analyzer. The Potentiostat tab is for controlling the 1287 when in $\mathrm{CV}$ mode and the Galvanostat tab is for controlling the 1287 in CC mode. The Analyzer tab controls the 1260 configuration.

In order to make an impedance response measurement, select the type of measurement ( $\mathrm{CV}$ or $\mathrm{CC}$ ) from the main window in ZPlot. The $\mathrm{CV}$ measurement is the default measurement type and is the "Ctrl E: Sweep Freq" tab. Similarly, the CC measurement is the "Ctrl I: Sweep Freq" tab. Then, after entering values for the 
controlled parameter (DC voltage or current), the $\mathrm{AC}$ amplitude, and the frequency range of the signal, the software is ready to begin taking measurements.

\subsubsection{Overview of ZView}

After the impedance response data has been collected by ZPlot, the data can be displayed in ZView. ZView can display previously saved data and it also can display the data in real time as it is being collected. The data can be plotted in several different formats, the most common being a Nyquist plot of the real and imaginary components of the impedance and a Bode diagram of the magnitude and phase information. ZView can also be used to model the impedance response with an equivalent circuit. This requires the user to input a circuit, with approximate values for the components. ZView can determine the best fit for all the components and will display the model fit alongside the data. An equivalent circuit does not have to be fit to measured experimental data; ZView can also be used to simulate the impedance response of any circuit the user may specify.

ZView can also be used to process the data after the experiment. There are several operations that can be performed on the experimental data. ZView can add or remove equivalent circuit components and, consequently, the impedance response will reflect the change in equivalent circuit. This is useful for removing ohmic losses from the data (also referred to as iR-corrected data). This can also be used to remove known measurement artifacts, such as inductance introduced from cables. ZView can also scale the data to any desired cell area. This is a convenient way to normalize data that may have come from several different cells.

\subsection{Testing}

This section describes the testing that was used to evaluate a PEMFC stack with EIS measurements. For this work, the PEMFC stack temperature set point was $70^{\circ} \mathrm{C}$, but the measured temperatures from the separator plates between the cells ranged from as low as $45^{\circ} \mathrm{C}$ at low currents up to $60^{\circ} \mathrm{C}$ at high currents. The reactant gases were supplied at atmospheric pressure for both the anode and cathode. The fuel was $99.99 \%$ pure hydrogen gas and was supplied at a flow rate of 4 Standard Liters per Minute (SLPM). This total flow was split evenly among the four cells by the valves described above such 
that each cell was supplied 1 SLPM. The hydrogen gas was humidified by bubbling through water at a temperature of $70^{\circ} \mathrm{C}$ before reaching the stack. Air served as the oxygen-containing gas and was supplied to the cell cathodes at a flow rate of 16SLPM through a common manifold. As a result of the manifold, air flow rates to each cell could not be independently specified beyond the total stack flow rate. However, for this work, it was assumed that air flow was approximately divided equally, resulting in an air flow to each cell around 4SLPM. The air was also humidified at $70^{\circ} \mathrm{C}$. The PEMFC stack was always operated at these conditions for the entirety of this work.

While all the above conditions were held fixed for this work, the parameter that was varied was the DC load current supplied by the fuel cell. In order to cover a range of operating currents, two configurations were required. The first is a common configuration used when making EIS measurements on a single cell [27,47]. In this setup, the impedance measuring equipment regulates the DC load current for the fuel cell as well as measuring the impedance response from the applied AC signal. In this case, the impedance equipment is measuring the exact current that is passing through the fuel cell. This setup is diagrammed in Figure 2.5.

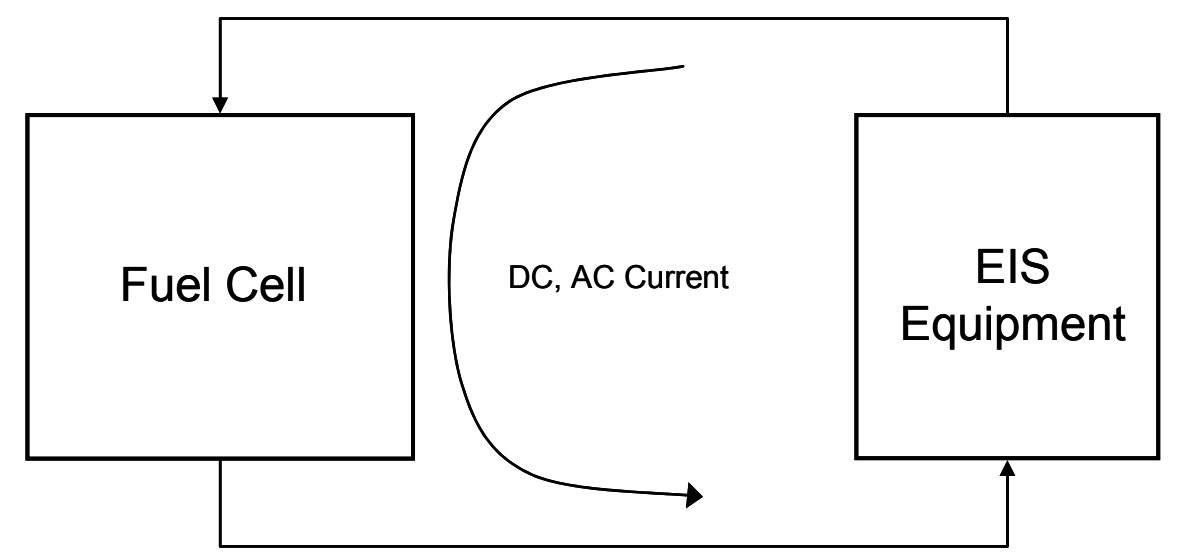

Figure 2.5: Low DC Current Setup

The other configuration involves connecting the Avtron load bank to the PEMFC to handle larger DC current while the impedance measuring equipment is connected in parallel to measure the $\mathrm{AC}$ response. This configuration has been demonstrated to work adequately on batteries and single cells [32]. The second setup is shown in Figure 2.6. 


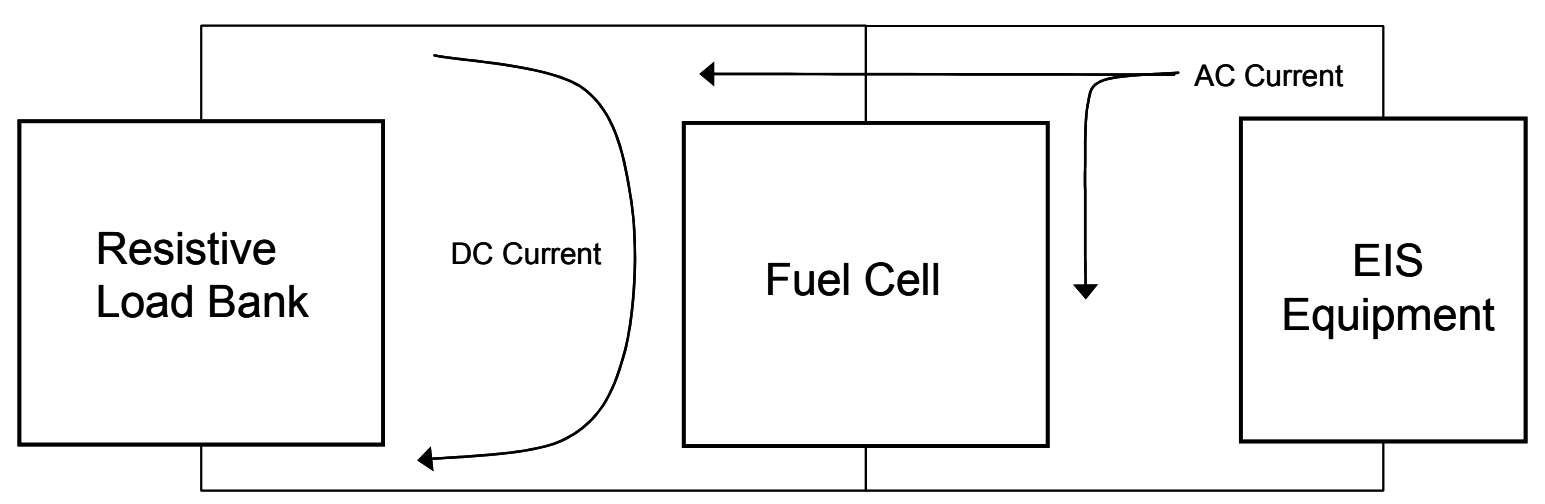

Figure 2.6: High DC Current Setup

\subsubsection{Connections}

There are three configurations that can be used when connecting the 1287's four leads to the fuel cell. These are two-, three-, and four-terminal measurements, which are described by the number of electrical connections that are made. The two-terminal configuration was used to perform the impedance response measurements on the PEMFC stack. The approach employed here involved passing the AC signal through the entire stack by connecting the $\mathrm{CE}$ and the WE leads to the current collector plates. The RE1 and RE2 leads were also connected to the current collector plates to measure the stack $\mathrm{AC}$ voltage induced from the applied AC current.

The polarity of the connections was such that the CE lead was attached to the stack anode current collector plate (lowest potential) and the WE lead was connected to the stack cathode current collector plate (highest potential). Both leads were attached using alligator clips to the tabs on the current collectors where the load bank cables connected. The reference electrodes were also attached to the current collector plates by alligator clips. The RE1 lead was connected to the same current collector plate as the $\mathrm{CE}$ lead. Similarly, the RE2 lead was connected to the same current collector plate as the WE lead. Refer to Figure 2.7, to see this schematically. 


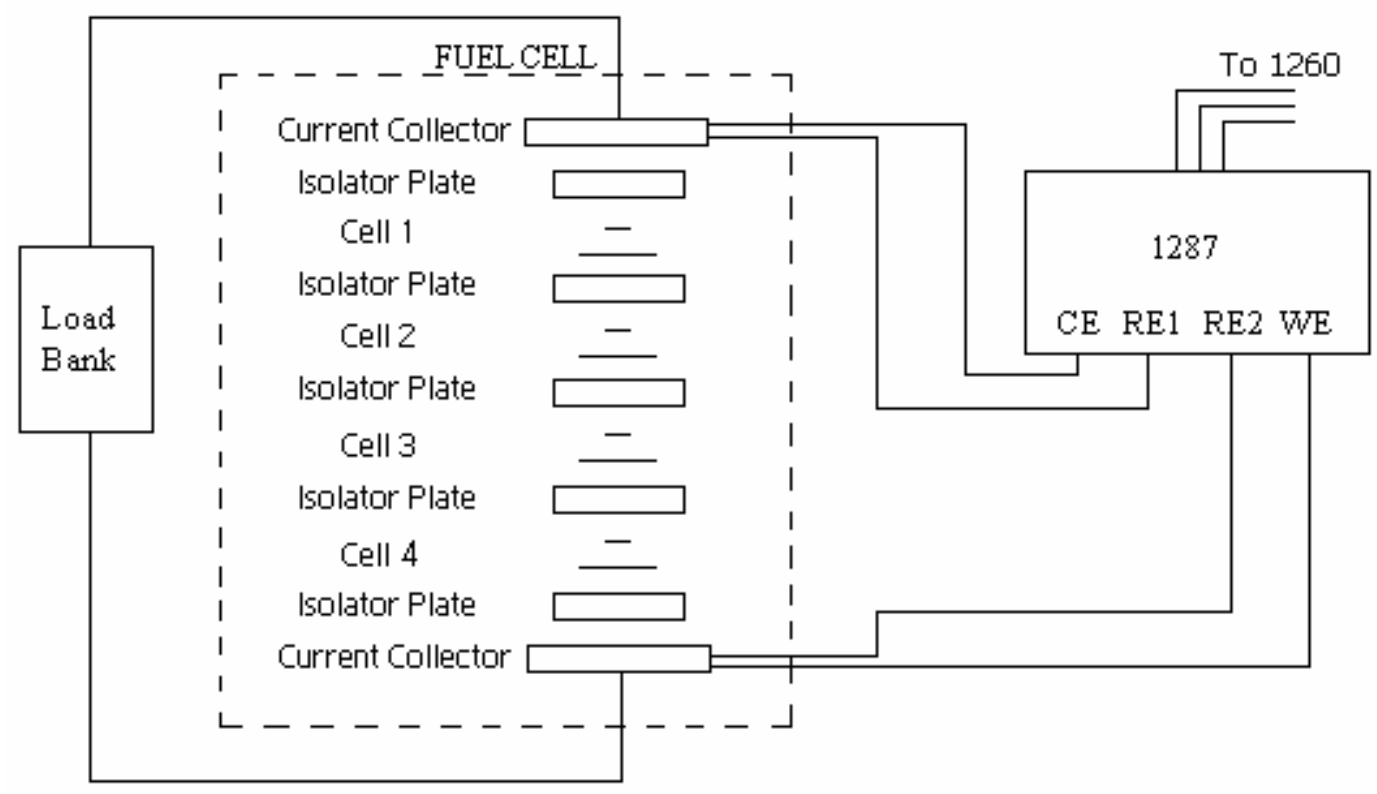

Figure 2.7: Two-Terminal Connection to PEM Fuel Cell using Solartron 1287

\subsubsection{Low Current Measurements}

Using the first configuration, most work previously reported has been performed on small single cells. These cells typically only have an area on the order of $1-5 \mathrm{~cm}^{2}$ $[25,26,27]$ With such a small cell, and gases at atmospheric pressure, it is easy to reach the limiting current density of approximately $1 \mathrm{Amp} / \mathrm{cm}^{2}$ without drawing much DC current. The $1287 \mathrm{ECI}$, however, is limited to a maximum of 2Amps of DC current, which is incapable of supplying enough current for the size of cells used in this work to reach the limiting current density. The cells, with an area of $134 \mathrm{~cm}^{2}$, would require approximately $130 \mathrm{Amps}$ to reach the limiting current density. Since this greatly exceeds the capabilities of the 1287, this setup was limited to DC currents less than 2Amps.

When running the first configuration, three DC current loads were selected to give some distribution in the available data. Since the operating region was from 0Amps up to $2 \mathrm{Amps}$, it was decided to use $0.2,1$, and $1.8 \mathrm{Amps}$ as the DC load current with an $\mathrm{AC}$ rms magnitude of $100 \mathrm{~mA}$. This provided a margin of $0.2 \mathrm{Amps}$ at both the lower and upper operating bounds to allow for the effects of the $\mathrm{AC}$ current. At low frequency $\mathrm{AC}$ current, the instantaneous current endures prolonged deviations from the $\mathrm{DC}$ value, up to a maximum offset of the $\mathrm{AC}$ current's magnitude. If the $\mathrm{AC}$ magnitude is too large, these oscillations can push the instantaneous current value outside of the valid operating 
region, currents between $0 \mathrm{ADC}$ and $2 \mathrm{ADC}$. At the lower bound, these oscillations could result in a negative instantaneous current, which would attempt to drive current through the fuel cell in the opposite direction. This could be potentially damaging to the MEAs. In the case of the upper bound, this means the instantaneous current could go above 2Amps for extended periods of time, which is not within the specifications of the Solartron equipment.

\subsubsection{High Current Measurements}

Due to the low current limit of the first configuration where the 1287 ECI served as the DC load, another setup was employed in order to measure the impedance response at higher load conditions. The high current setup involved connecting an Avtron load bank to the PEMFC in order to draw more DC current. The Avtron load bank allowed DC currents of up to approximately 67Amps, or a current density near $0.5 \mathrm{~A} / \mathrm{cm}^{2}$. This is well short of the load bank's full scale current rating of 120Amps. This is due to the fact that the load bank has a specific DC Voltage rating. As described previously, the load bank is rated for $3 \mathrm{~V}$ operation, meaning that all the resistive elements are designed to only draw the rated current if a $3 \mathrm{~V}$ potential is applied across the terminals. However, the PEMFC stack voltage does not maintain a constant value, but rather, generally decreases with increasing current. This behavior is shown in a Voltage-Current Polarization curve (VI curve) as shown in Figure 2.8. 


\section{Curve}

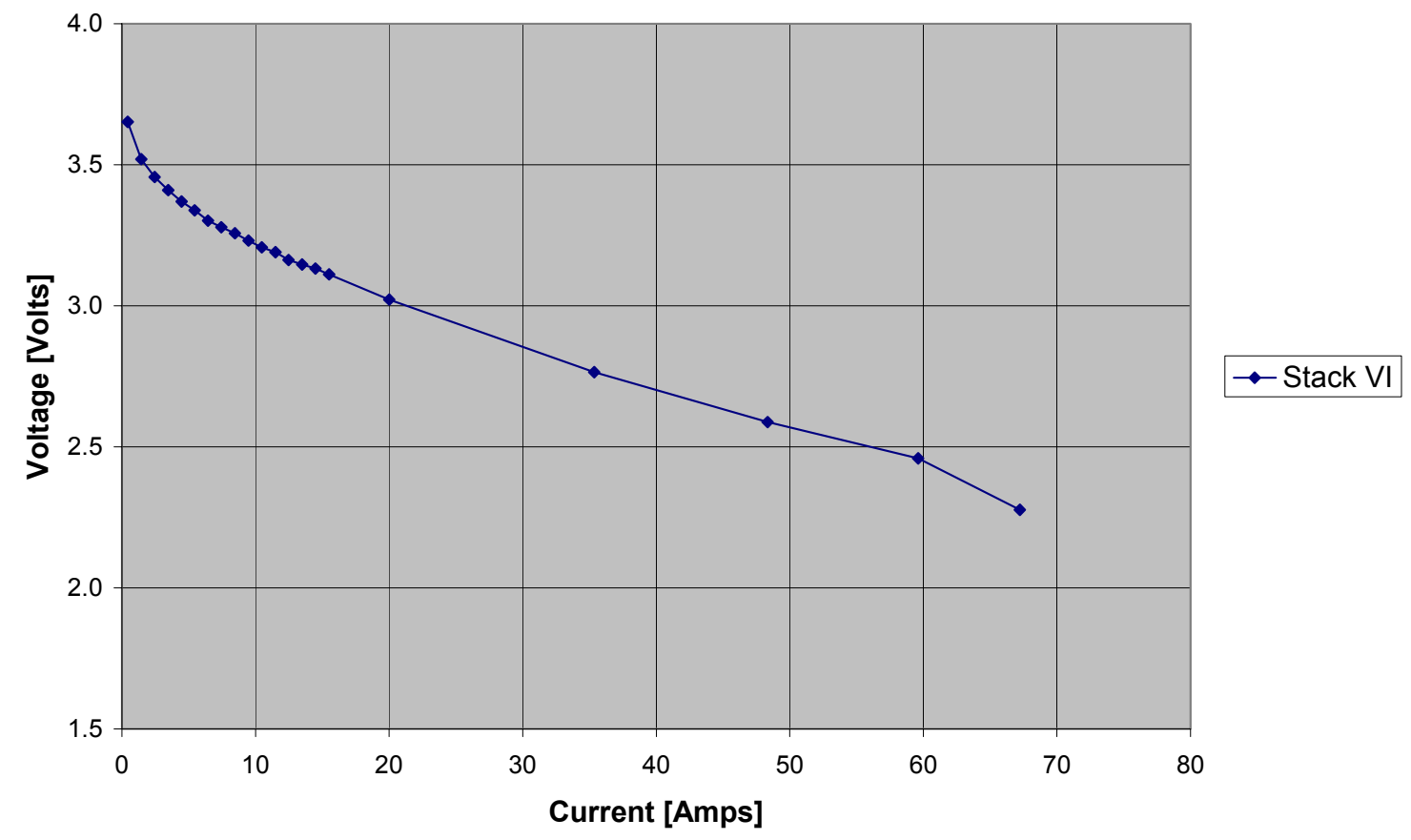

Figure 2.8: Typical VI curve for the PEMFC stack

It can be seen that the stack voltage is above 3 volts until a DC current of 20Amps is reached. At this point, there are enough losses to bring the stack voltage low enough to be able to properly drive the Avtron load bank. At currents above 20Amps, the stack voltage continues to decrease as more losses are subtracted from the open circuit potential. This effect continues to decrease the PEMFC's ability to drive current through the Avtron load bank as the stack voltage increasingly deviates from the nominal 3 Volt rating.

It should be noted that the voltage measured separately by the Avtron load bank was not the same as the voltage recorded for the VI curve. The Avtron systematically measured a voltage which was lower than the VI data. The difference in the voltages appeared to be proportional to current. Table 2.1 summarizes the load settings used on the Avtron and the corresponding DC current. The different voltages measured are also listed, with the percent error based on the measured current. 
Table 2.1: Comparison of Current Setting and Actual Current

\begin{tabular}{|c|c|c|c|c|}
\hline \multicolumn{2}{|c|}{ Load Bank Setting } & Current Measured & \multicolumn{2}{c|}{ Stack Voltage [Volts] } \\
\hline [Amps] & {$[$ Ohms] } & [Amps] & VI Data (\%error) & Avtron (\%error) \\
\hline 20 & 0.15 & 20 & $3.02(<1 \%)$ & $3.0(<1 \%)$ \\
40 & 0.075 & 35 & $2.76(5.1 \%)$ & $2.7(2.9 \%)$ \\
60 & 0.05 & 48 & $2.59(7.9 \%)$ & $2.5(4.2 \%)$ \\
80 & 0.0375 & 59 & $2.46(11 \%)$ & $2.3(4.0 \%)$ \\
100 & 0.03 & 67 & $2.28(13 \%)$ & $2.1(4.3 \%)$ \\
\hline
\end{tabular}

\subsubsection{Parallel Load Effects}

As a consequence of connecting the load bank to the PEMFC stack, the circuit being examined with EIS is no longer just the PEMFC stack. The impedance response will be the parallel combination of both the PEMFC stack and the Avtron load bank. In order to find the isolated PEMFC stack response at higher current loads, first, the total combined response was measured. Then, the load cables, which connect the Avtron load bank to the PEMFC current collectors, were disconnected from the current collector plates. An impedance response was then taken of the load bank and cables by connecting the impedance equipment to the free end of the load cables. This response was then subtracted from the combined response to determine the isolated PEMFC stack impedance response.

At each of the current loads, in order to calculate the PEMFC response from the measured combined response, the following equation was used to relate the combined and load responses to the PEMFC stack response,

$$
\frac{1}{Z_{\text {Stack }}(j \omega)}=\frac{1}{Z_{\text {Combined }}(j \omega)}-\frac{1}{Z_{\text {Load }}(j \omega)}
$$

This calculation was performed in MATLAB using two M-files. One to read the ZView impedance data files of the combined response and the isolated load response ('readzfile.m'). The other file performed the calculation and returned the isolated PEMFC stack response ('subtractload.m'). Both files are listed in the Appendix. 


\subsection{Results}

This section presents the results of the experimental work. This shows the impedance spectrum taken on an operating PEMFC stack.

\subsubsection{Low Current Conditions}

First, the low current measurements were taken using the setup shown in Figure 2.5. It is generally recommended [24] that the magnitude of the AC current be set to approximately $1 \%$ of the DC value passing through the fuel cell. For these low current measurements, the recommended value would be on the order of $10 \mathrm{~mA}$ (for the $1 \mathrm{~A}$ case). However, there was a significant level of noise in the impedance spectra and a much larger value of $100 \mathrm{~mA}$ was needed to generate a smooth curve with less noise in the data. This value was used for all three low current cases, resulting in an $\mathrm{AC} / \mathrm{DC}$ ratio ranging from 0.5 to 0.0555 for the DC currents $0.2 \mathrm{~A}$ and $1.8 \mathrm{~A}$, respectively. This did not appear to violate the linearity requirement which is necessary for all EIS measurements. The low current EIS response is shown in Figure 2.9. The frequency range is $10 \mathrm{kHz}-$ $0.025 \mathrm{~Hz}, 10$ steps per decade, with the high frequency plotted toward the left side of the plot.

From the plot of the low current response, there only appears to be one arc in all the cases. This arc is not a true semicircle, but is slightly depressed, meaning that the base is greater than two times the height. A true semicircle would have a base (diameter) that is twice the height (radius). This arc also appears to be, at least initially, highly dependent on current. There does not appear to be a change in ohmic resistance as all

three curves have approximately $1 \Omega-\mathrm{cm}^{2}$ high frequency resistance, as can be seen in Figure 2.11, which shows a close-up of the high frequency content. 


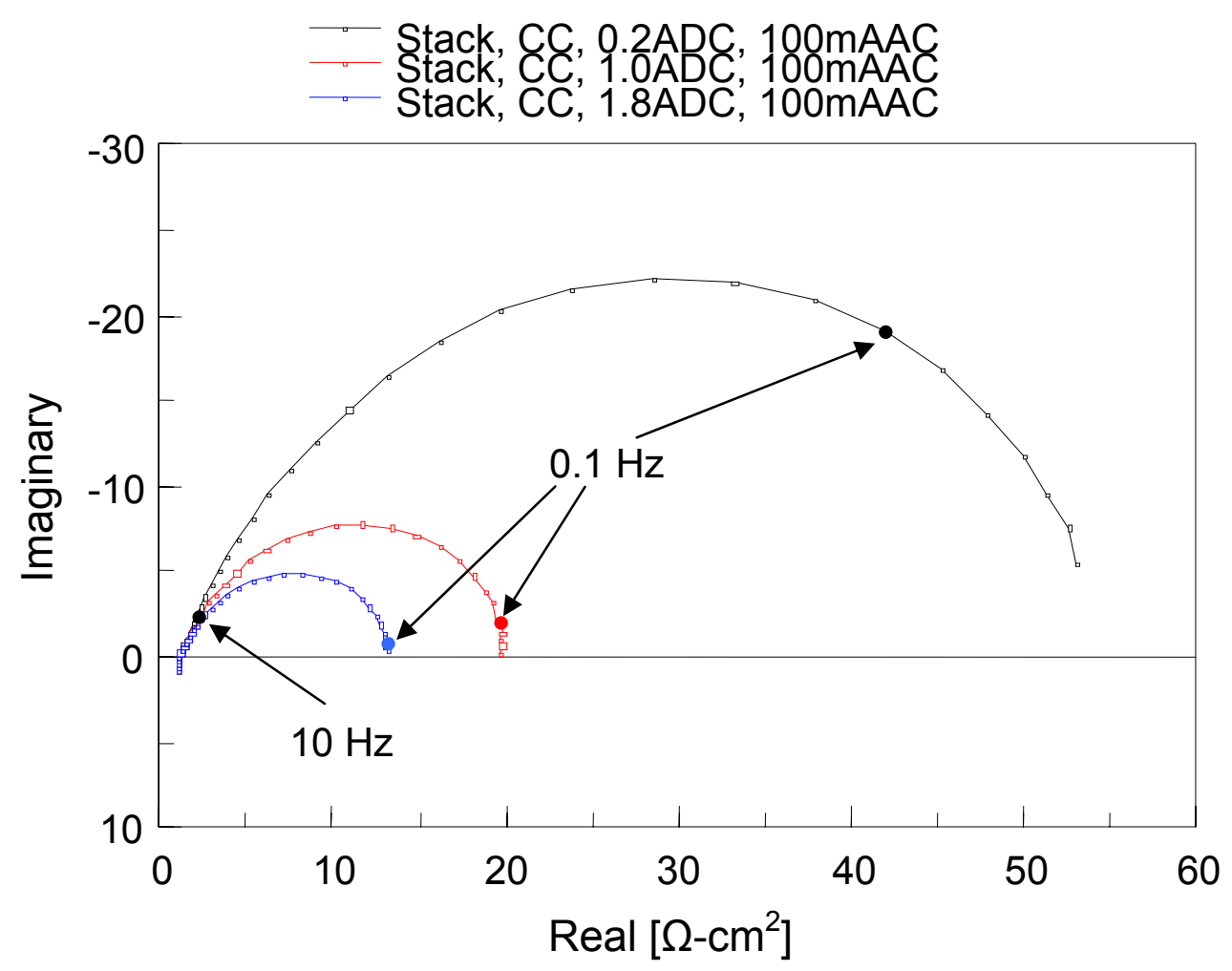

Figure 2.9: Low Current EIS Response
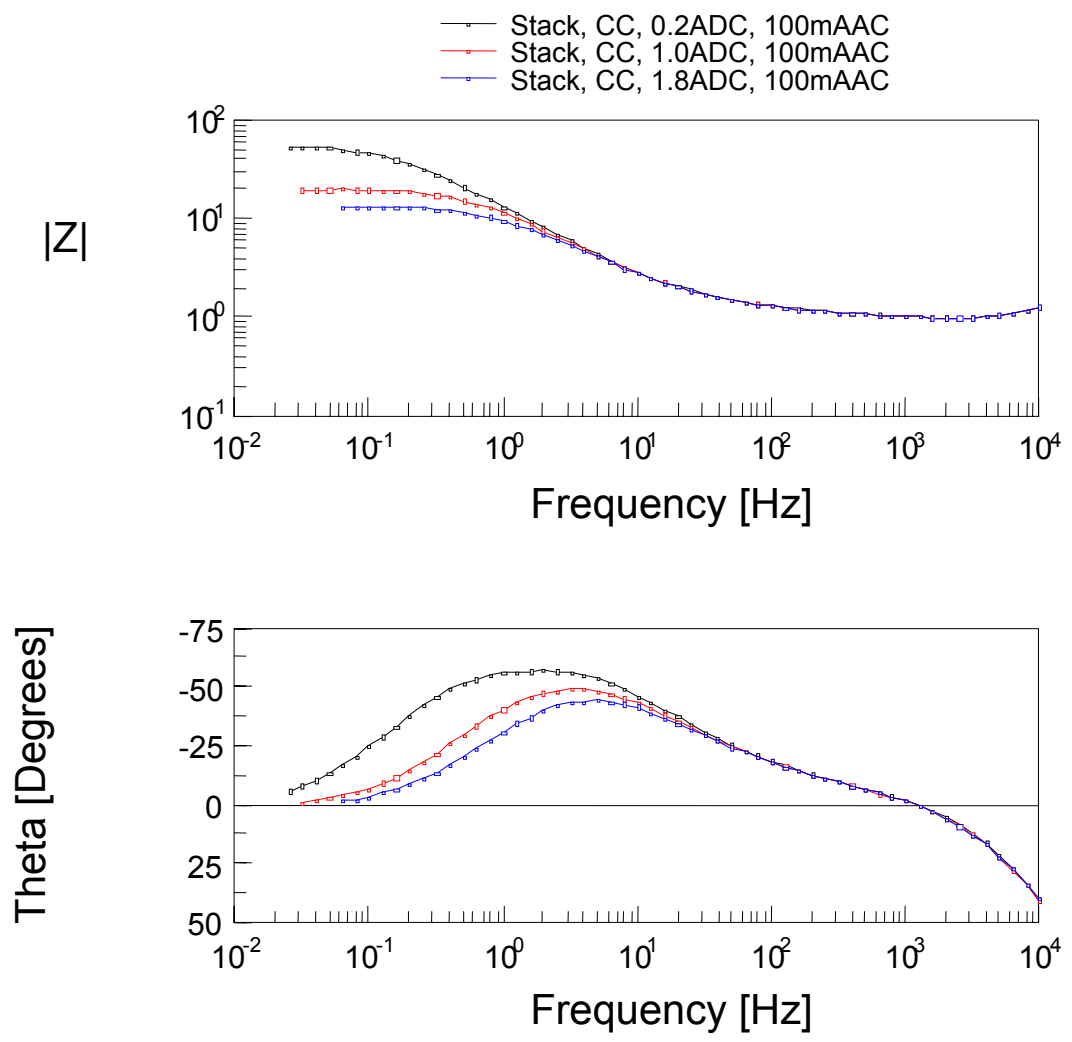

Figure 2.10: Bode Plot of Low Current Response 


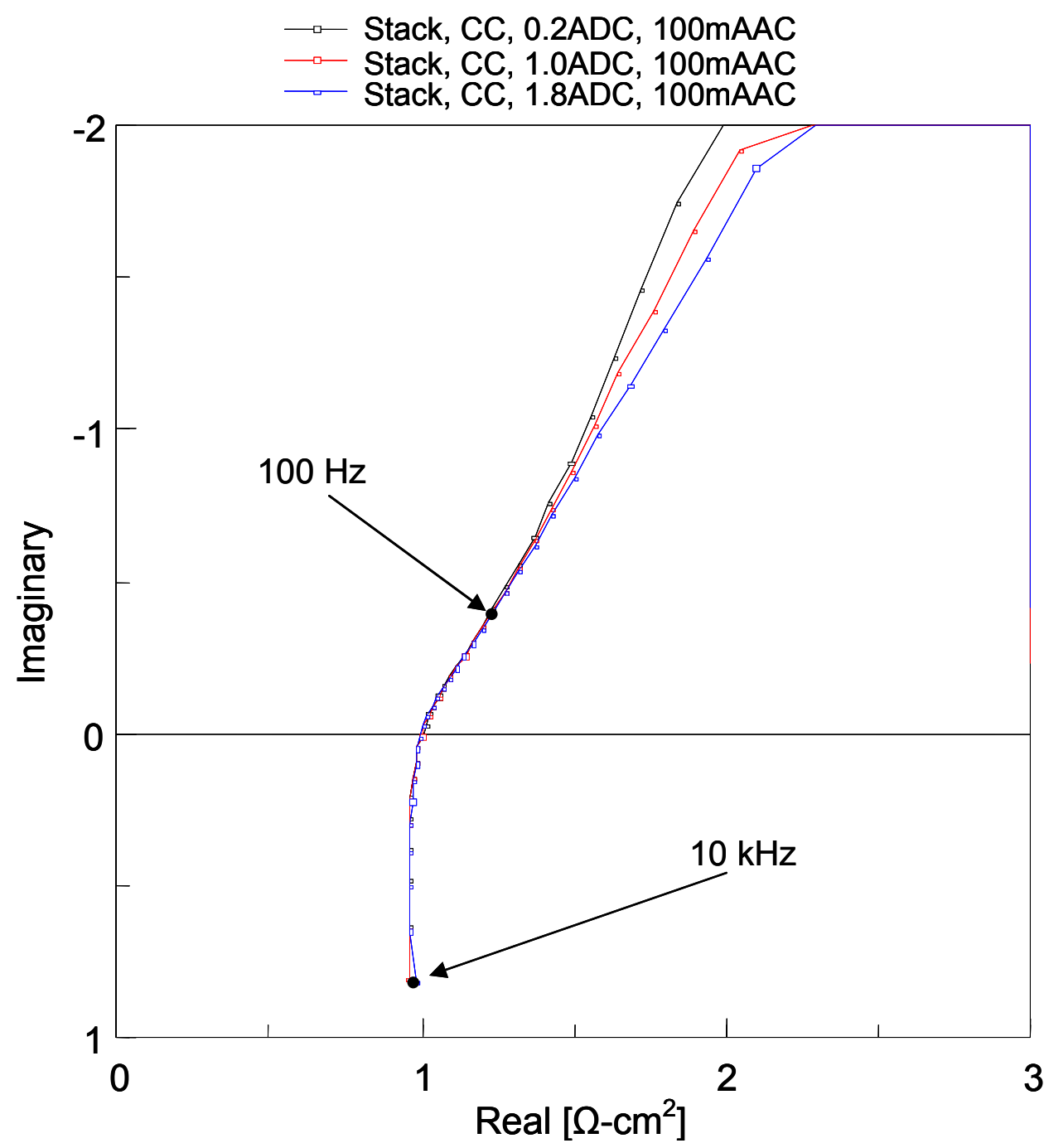

Figure 2.11: High Frequency of Low Current EIS Response

The inductance present at the highest frequencies is common in low impedance electrochemical devices such as batteries and fuel cells [24]. This inductance is usually attributed to the cables and connectors that are used when making the measurements.

\subsubsection{High Current Condition}

The higher current measurements used the Avtron load bank so the fuel cell could be operated at DC currents not attainable by using only the Solartron impedance equipment. As a consequence of this setup, the measured impedance response was the total response of the parallel combination of the fuel cell and the load. Similar to the low current measurements, there was a significant amount of noise in the impedance response if a small AC magnitude ( $\sim 1 \%$ of DC magnitude) was used. It was found that an $\mathrm{AC}$ 
magnitude of $1 \mathrm{~A}$ produced a smooth curve until the lowest frequencies, where it is very difficult to remove the noise. Figure 2.12 shows the results of three different AC magnitudes, $200 \mathrm{~mA}, 500 \mathrm{~mA}$, and $1000 \mathrm{~mA}$, on the measured stack response at a DC current load of 20A.

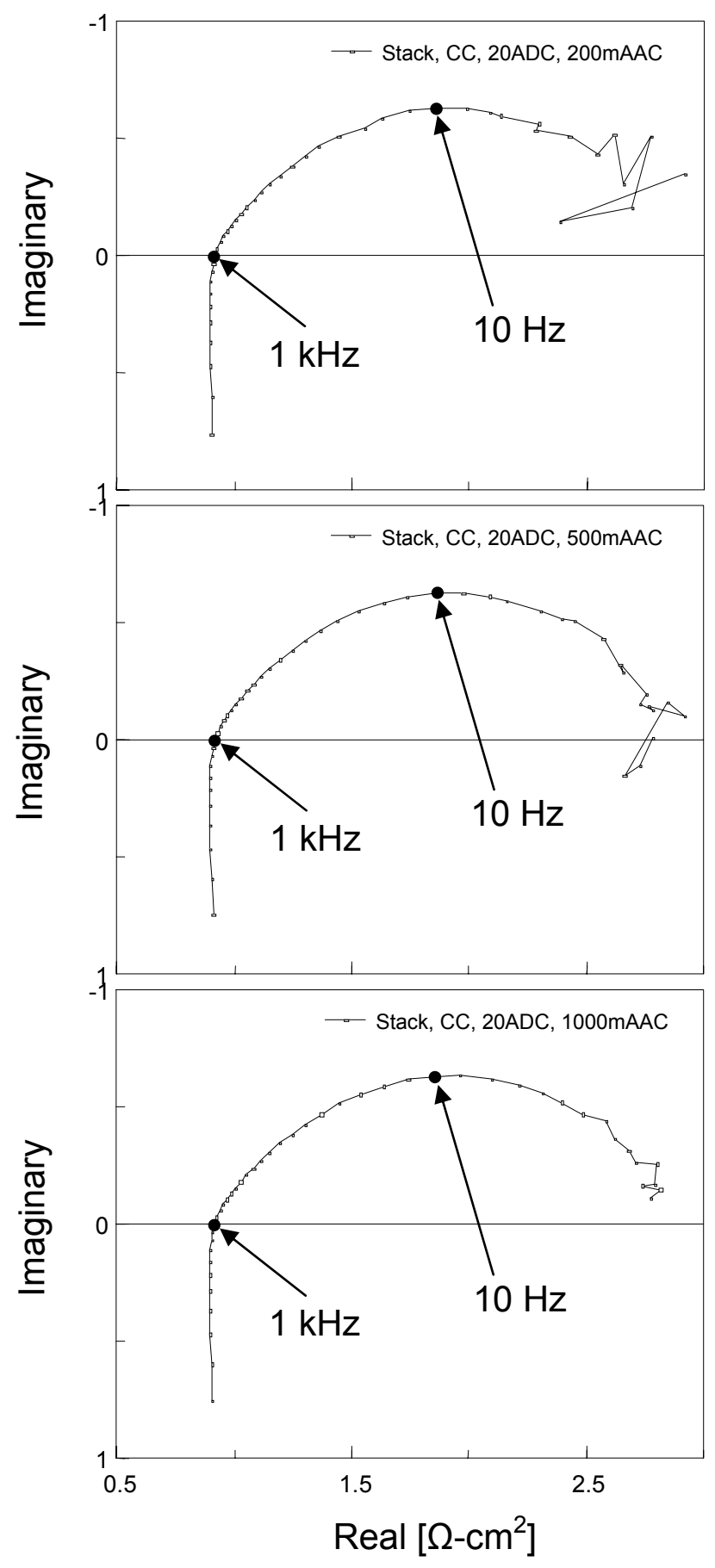

Figure 2.12: Effect of Changing AC Magnitude at 20 Amps Load 
Using the 1A AC signal, the PEMFC was operated at various DC current loads and the impedance response was measured at each load. The DC currents used to drive the PEMFC were described in Section 2.3. As the DC current increased, thus reducing the $\mathrm{AC} / \mathrm{DC}$ current magnitude ratio, the responses generally became noisier. At the highest two DC current loads, 59 and 67 Amps, the magnitude of the AC current was increased to $1.333 \mathrm{~A}$. This value is close to the largest AC current that can be used when the measurement requires low frequency information $(<10 \mathrm{~Hz})$, even though it is still well beneath the absolute $2 \mathrm{~A}$ current limit of the 1287 ECI. The reason is the specified value of the AC magnitude (1333mA) is the root mean square (rms) magnitude, which is actually the maximum instantaneous current, divided by the square root of two. At high frequencies $(>10 \mathrm{~Hz})$, this is not an issue, but at low frequencies, when $\mathrm{AC}$ begins to approximate DC, the instantaneous current must not exceed the limit of the 1287. The measured responses at each current are shown below in Figure 2.13. 


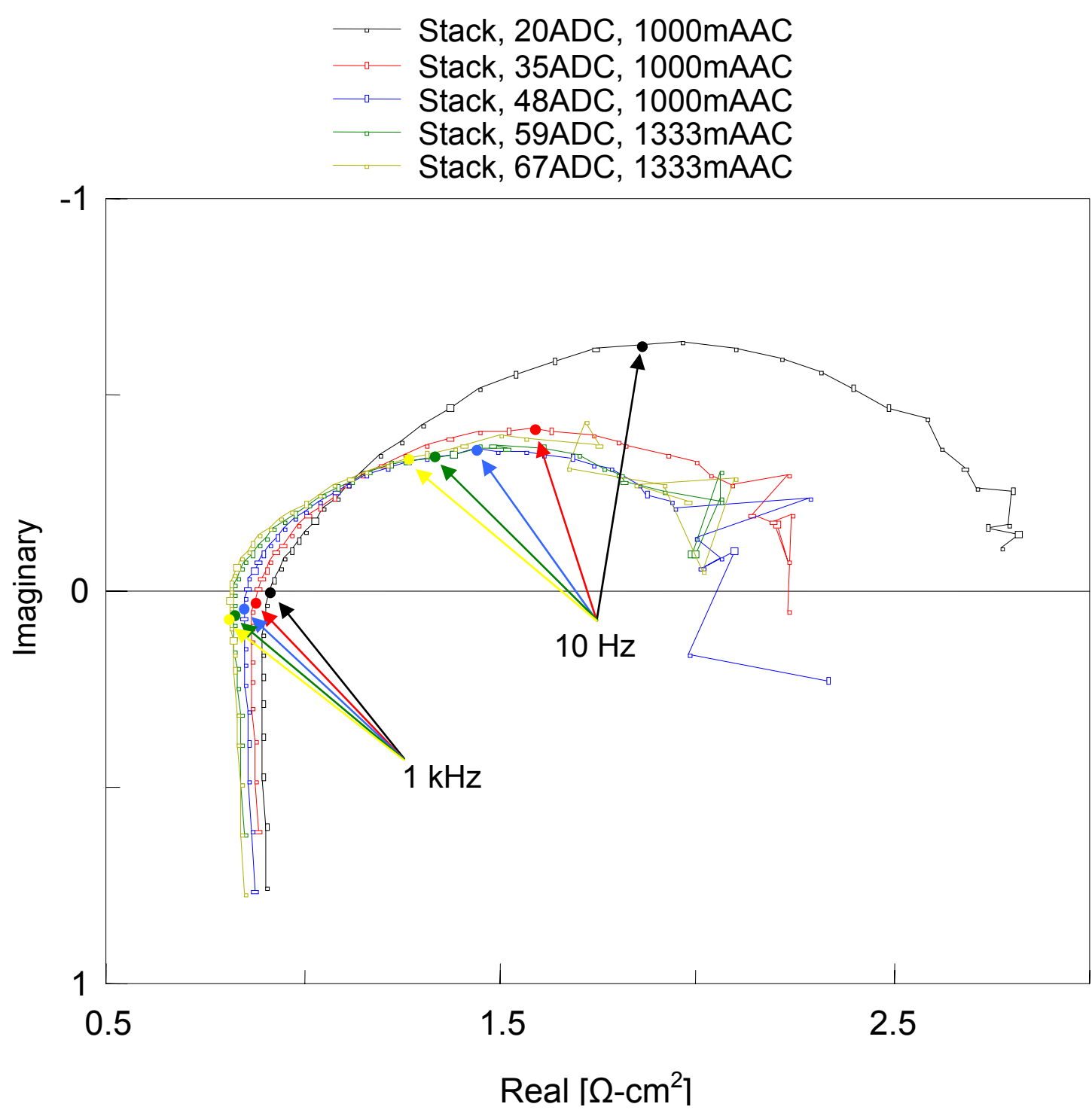

Figure 2.13: Measured Total Impedance Response

In order to determine the isolated response of the PEMFC stack, it was necessary to measure the load separately. Each load that was applied to the PEMFC stack was measured separately and is shown in Figure 2.14. The positive imaginary component shows that the load bank was not an ideal resistor, but showed some inductance was present in the resistive elements. 


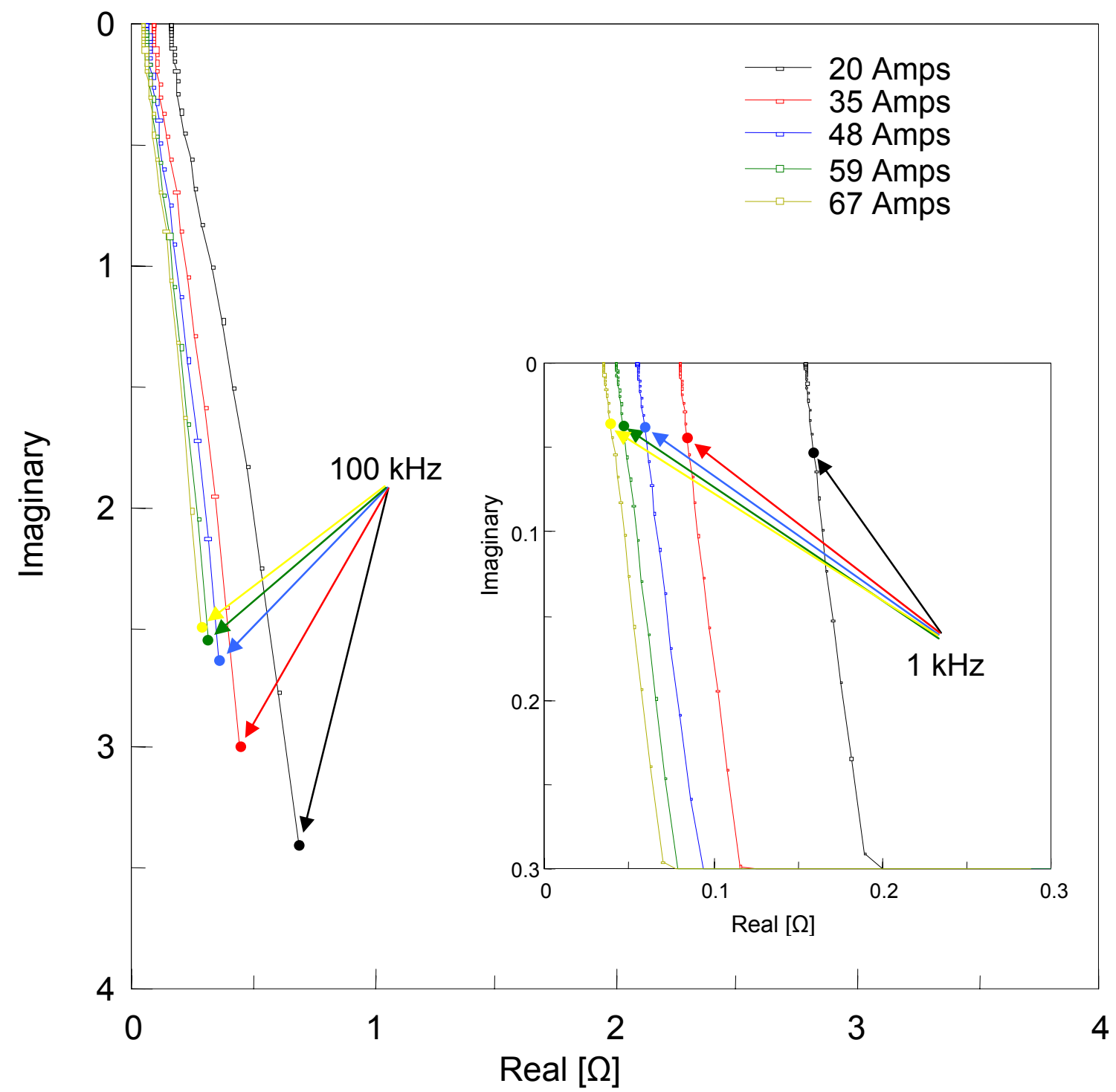

Figure 2.14: Impedance Response of Avtron Load at Various Currents (Inset: Close up of Low Frequency)

Finally, to determine the isolated PEMFC stack response, Equation 2.1 is applied to the total measured response data in order to remove the effects from the parallel load. To illustrate the effect the load had on the measured response, both measured and calculated responses of the 20 Amp case are shown in Figure 2.15. All of the corrected PEMFC stack responses are shown in Figure 2.16. 


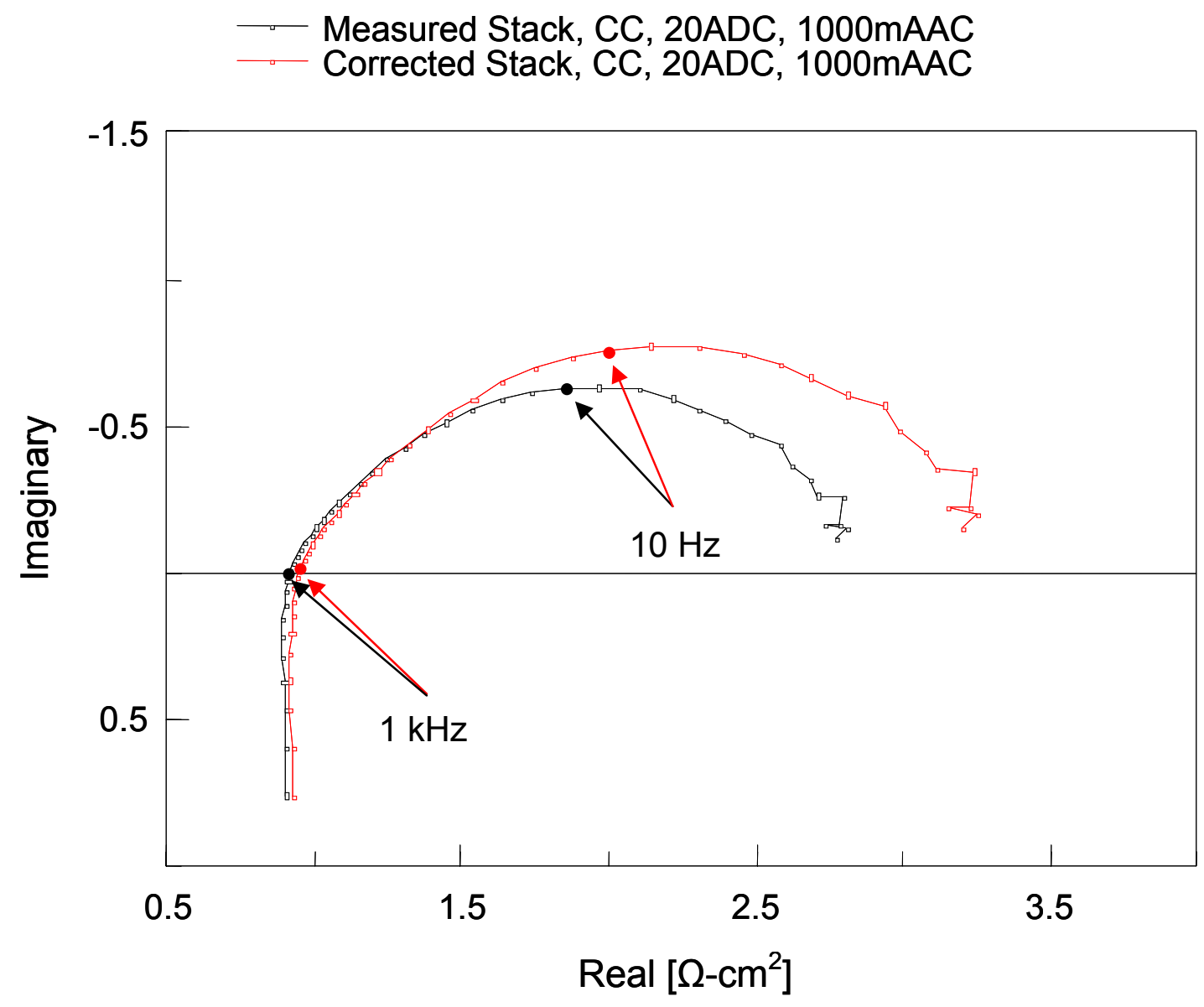

Figure 2.15: Load Correction Applied to 20A Measurement 


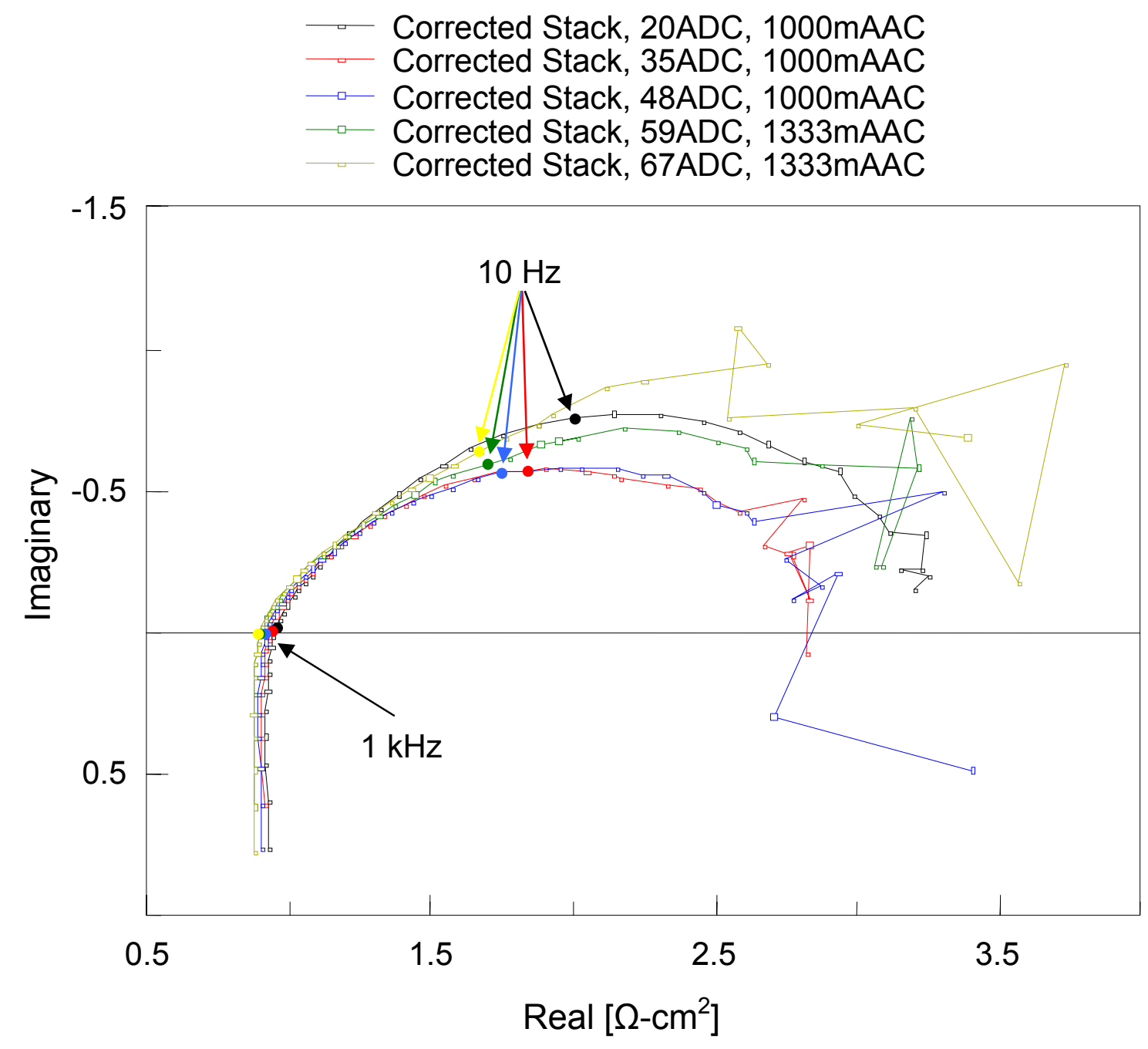

Figure 2.16: Corrected PEMFC Stack Response

A discussion of the experimental results will be presented in Chapter Four, which will compare the data to the model simulations. The next chapter will describe the MATLAB model used to simulate the PEMFC. 


\section{Chapter 3: Model}

A model of the four cell PEMFC stack was developed to simulate the behavior of the fuel cell at different DC current loads. A goal of the model was to provide assistance in interpreting the experimental results. The model was designed to simulate the behavior of the four cell PEMFC stack in terms of matching the V-I curve as well as the EIS measurements. The model was not intended to provide a perfect match to the experimental data, but rather show the relationships and trends that are present in the experimental data as the DC current changes. The dynamic fuel cell model was developed using MATLAB ${ }^{\circledR} /$ Simulink $^{\circledR}$.

\subsection{Model Description}

This section describes the fuel cell model including the basic assumptions made regarding the form and functionality of the model, the general layout and block diagrams, and the equations used to describe the fuel cell behavior. Overall, the model is designed to be a simplified bulk representation of a Hydrogen/Air PEMFC stack and associated support systems and is based on the model in Gemmen [48].

\subsubsection{Model Assumptions}

The model assumes uniform temperature distribution throughout all of the cells and the stack components. The temperature is also assumed to remain constant during operation. The cell current density is also presumed to be uniformly distributed through the active area of the cells. At the cathode, the porous electrode is believed to remain free of condensation of product water. Therefore, cathode flooding, which increases diffusional losses, is not considered at present.

The model also assumes three loss mechanisms - electrochemical activation loss, ohmic loss, and diffusion loss. The electrochemical activation loss is determined by the Butler-Volmer equation, a chemical rate equation relating charge-transfer and potential. The ohmic loss is, in reality, a sum of many resistive losses as mentioned previously. However, for simplicity, the model lumps all resistive losses together into a single resistance responsible for the ohmic loss. Finally, the model attributes the diffusion loss 
to gas diffusion through the porous gas diffusion layer for both anode and cathode. All the equations used to replicate these loss mechanisms are discussed in more detail later in the Equations section.

\subsubsection{Model Layout}

The fundamental systems represented are the fuel cell stack, the electrical load, the gas humidifiers, and a basic control system based on fuel utilization. The overall system block diagram is shown in Figure 3.1

\section{PEM Fuel Cell System}

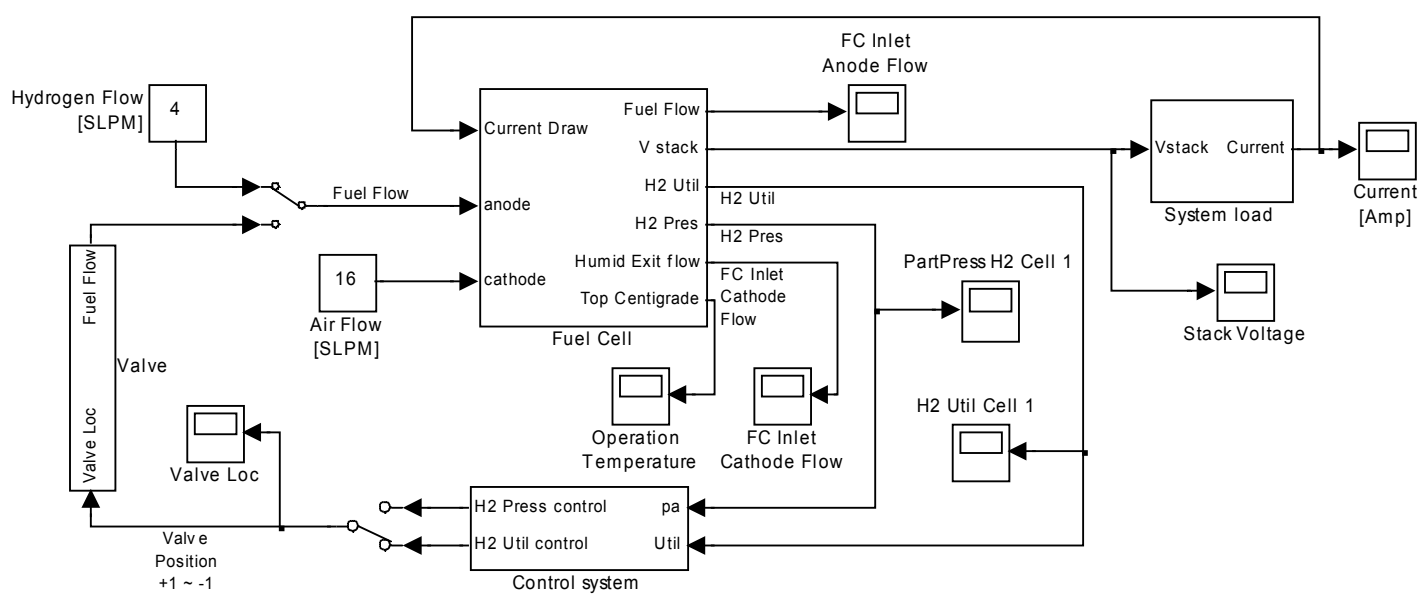

Figure 3.1: PEMFC System Block Diagram

The Fuel Cell block of Figure 3.1 contains the PEMFC stack as well as the gas humidifiers. The Fuel Cell block detail is shown in Figure 3.2. The gas humidifiers for the hydrogen and air flows are in the upper left of the diagram. The stack in Figure 3.2 is represented by the collection of four individual cells in the center of the diagram. The outputs of the individual cells are colored according to cell number.

The individual cell blocks of Figure 3.2 are identical copies of the same cell and all behave in exactly the same manner. The same result could easily have been attained by multiplying the output from a single cell block by the number of cells in the fuel cell stack. The model was implemented with four separate but identical cells to more closely match the experimental setup already described in Hensel, et al. [44] and thereby facilitate future modeling work when the fuel flow to each cell is not uniform. 


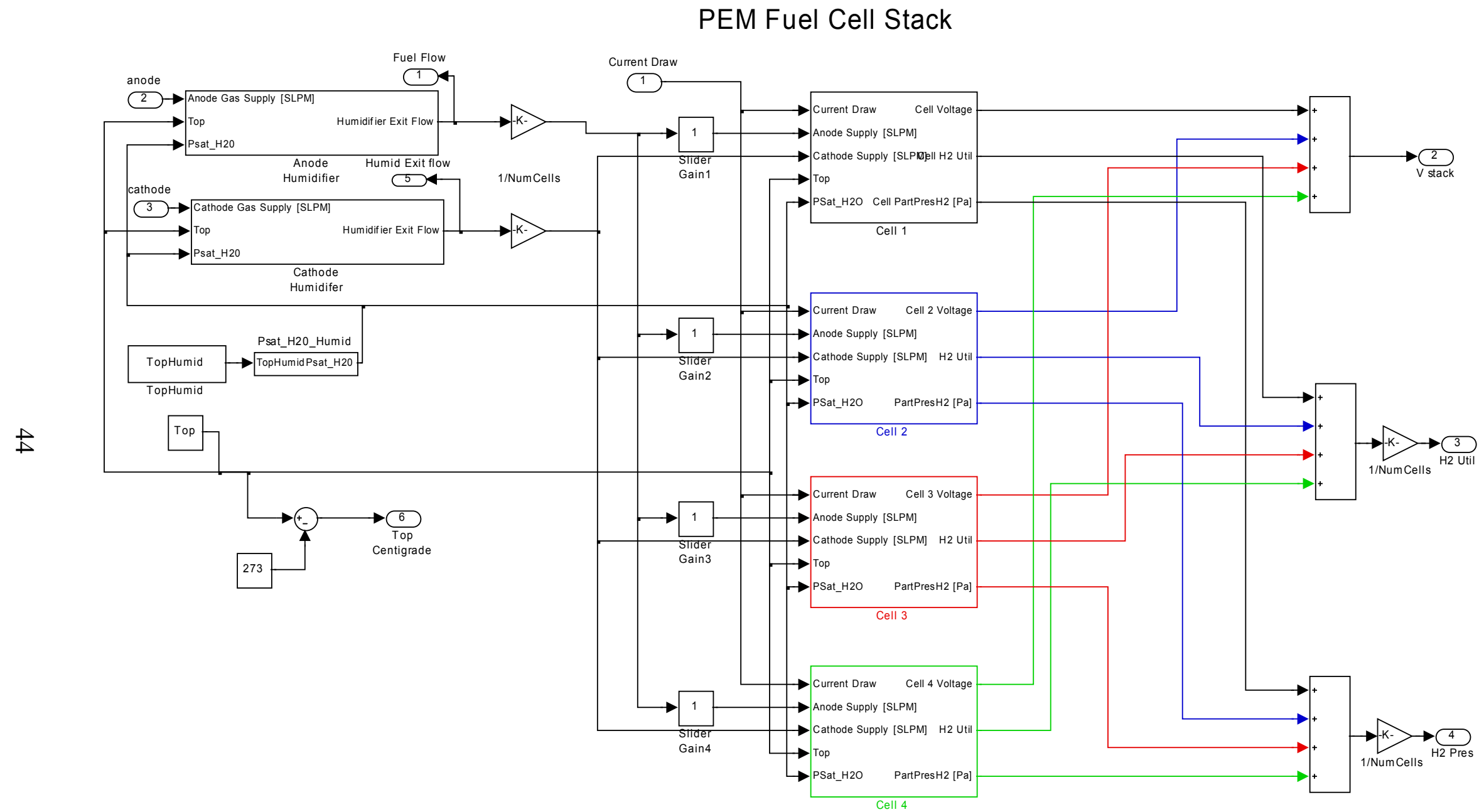

Figure 3.2: Fuel Cell Block Diagram 
The humidifier blocks in the model account for the extra flow in the gas streams created by the addition of water vapor to the reactant gases. The humidifier blocks can be seen in Figures 3.3 and 3.4. These humidified gases are then distributed to the cells in the stack. Both gas streams are divided equally to each of the cells.

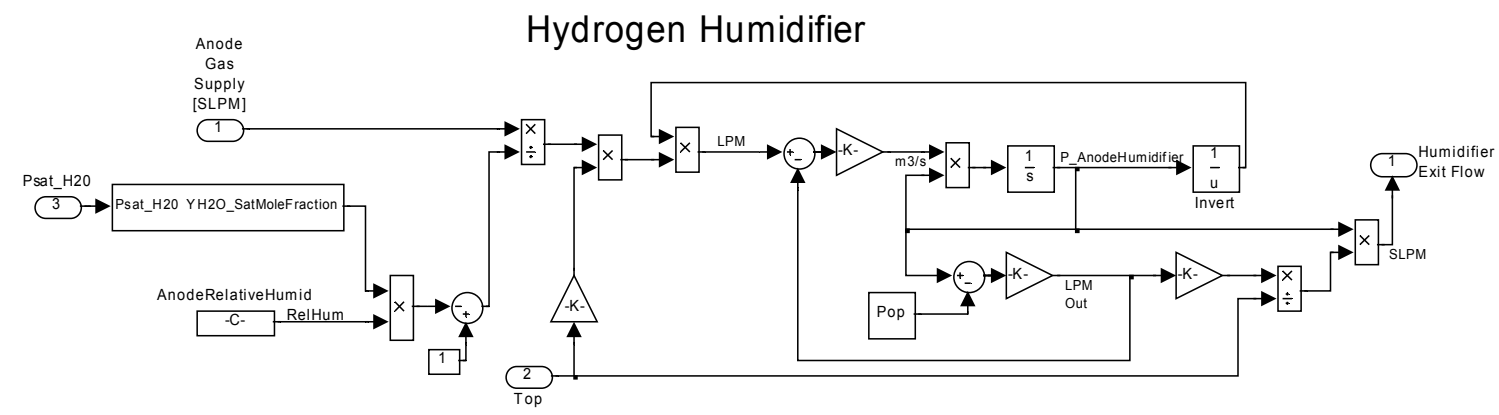

Figure 3.3: Hydrogen Humidifier Block Diagram

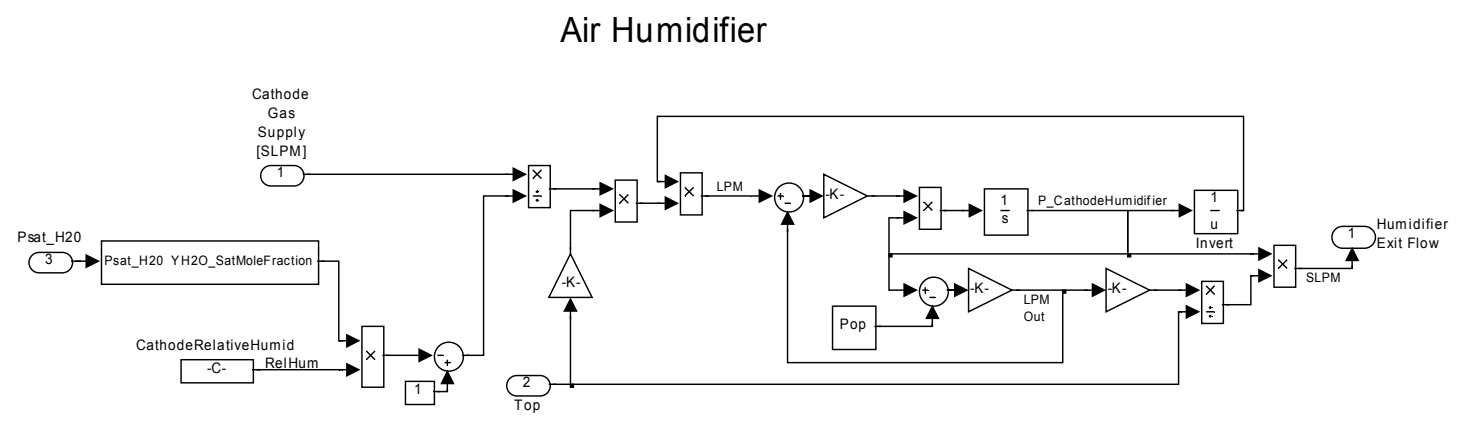

Figure 3.4: Air Humidifier Block Diagram

The Cell block of Figure 3.2 accounts for most of the calculations performed within the model and is diagrammed in Figure 3.5. These calculations include the consumption of hydrogen gas from the fuel flow at the anode, and the consumption of oxygen from the air flow at the cathode. This is also directly related to the production of water at the cathode. These parameters are determined within the Anode $\mathrm{H}_{2}$ Conservation and Cathode $\mathrm{O}_{2} / \mathrm{H}_{2} \mathrm{O}$ Conservation blocks, respectively. The diagrams for these blocks are shown in Figures 3.6 and 3.7. 


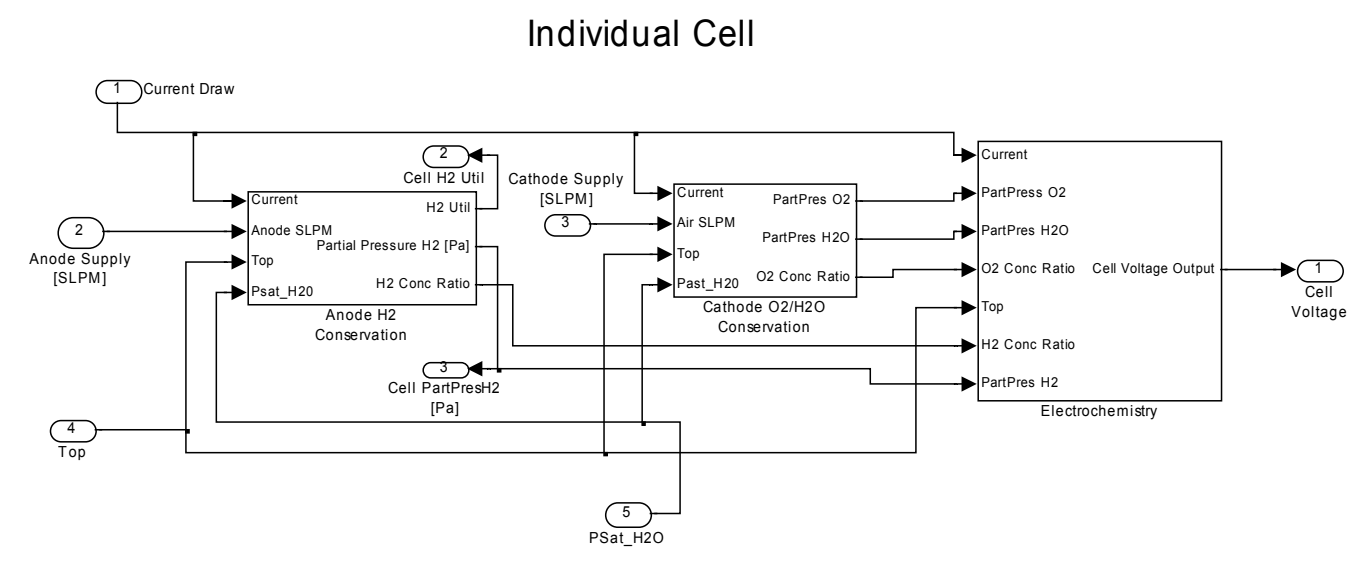

Figure 3.5: Individual Cell Block Diagram

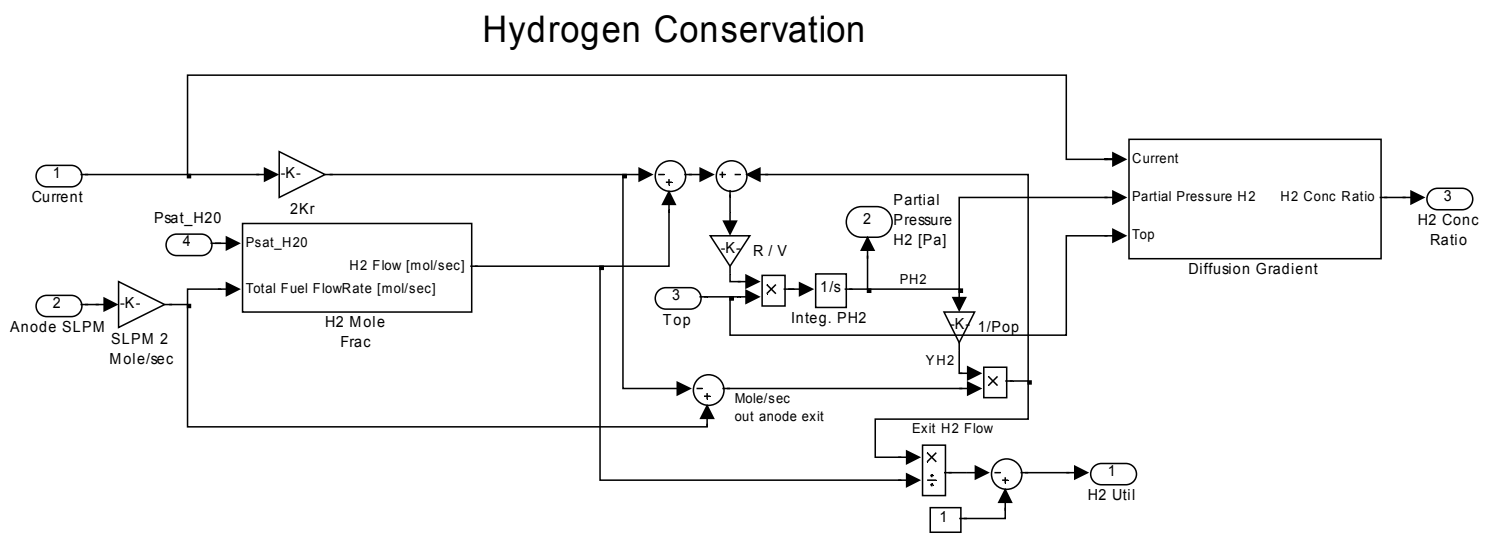

Figure 3.6: Anode Hydrogen Conservation Block Diagram 


\section{Oxygen / Water Conservation}

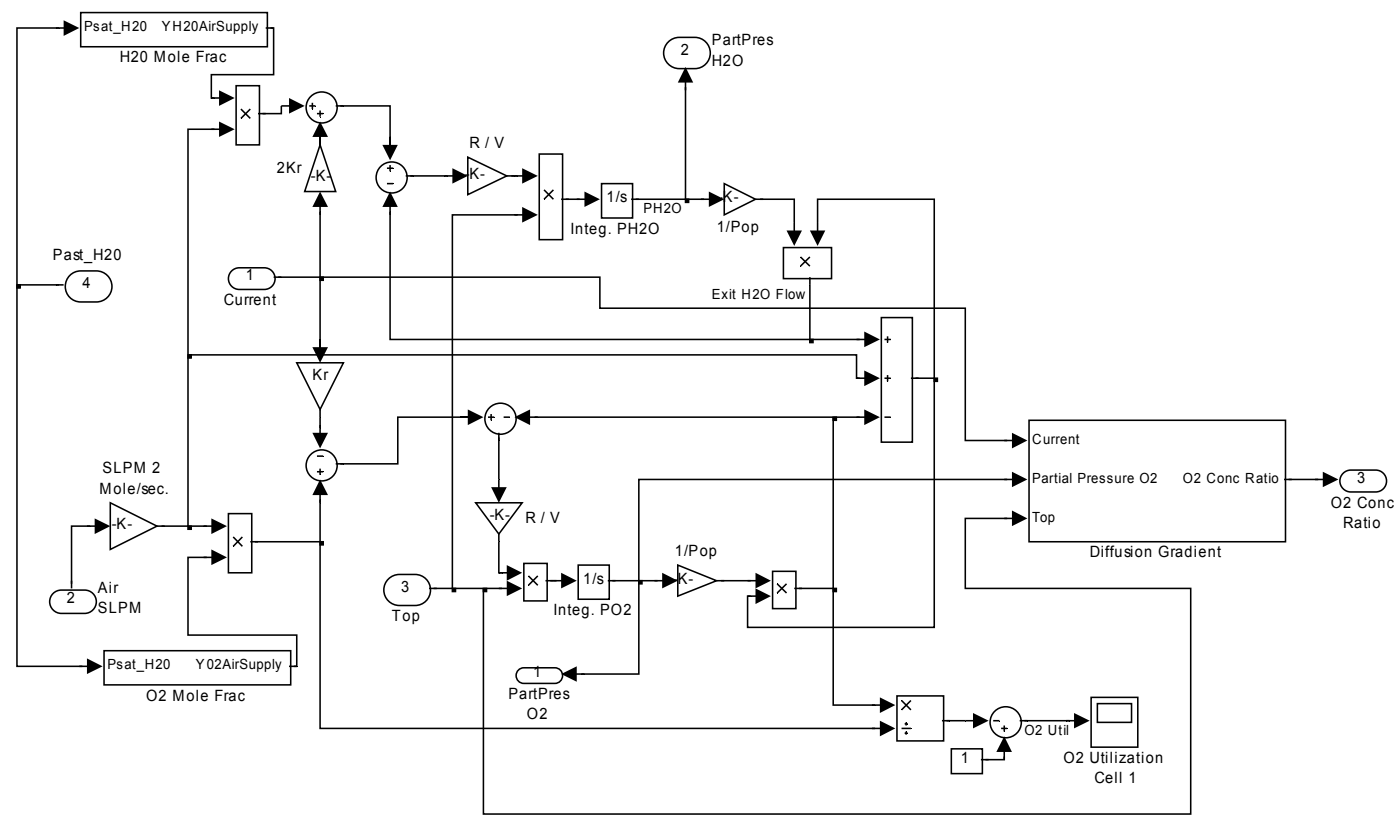

Figure 3.7: Cathode Oxygen / Water Conservation Block Diagram

The Cell block also calculates the electrochemical parameters, which is the heart of the fuel cell. These calculations are done in the Electrochemistry block, which is shown in Figure 3.8. The electrochemical calculations consist of the three main loss mechanisms which ultimately are responsible for the behavior of the VI curve, the electrochemical activation losses, the ohmic losses, and the diffusional losses. The calculation of the activation losses is done in the Activation Overpotential block shown in Figure 3.9.

It is important for the electrochemical calculations to use the gas concentration at the catalyst layer, which is where the reaction actually occurs, rather than the concentration at the flow channel. The change in gas concentrations from the free stream concentration at the serpentine flow channel to the catalyst layer concentration is calculated in the Anode $\mathrm{H}_{2}$ Conservation and Cathode $\mathrm{O}_{2} / \mathrm{H}_{2} \mathrm{O}$ Conservation blocks under the subsystem labeled Diffusion Gradient. The Diffusion Gradient block for the anode $\mathrm{H}_{2}$ concentration is detailed in Figure 3.10. Here, the gas concentration is calculated after considering the effects of the gas passing through the porous gas diffusion electrode. This concentration is then compared to a known concentration, similar to what was done by Bernardi [49]. The justification for this calculation is discussed below in the 
Equations section. The diffusion gradient calculation for the cathode $\mathrm{O}_{2}$ concentration is done the same way, with a different reference concentration value, and is not shown.

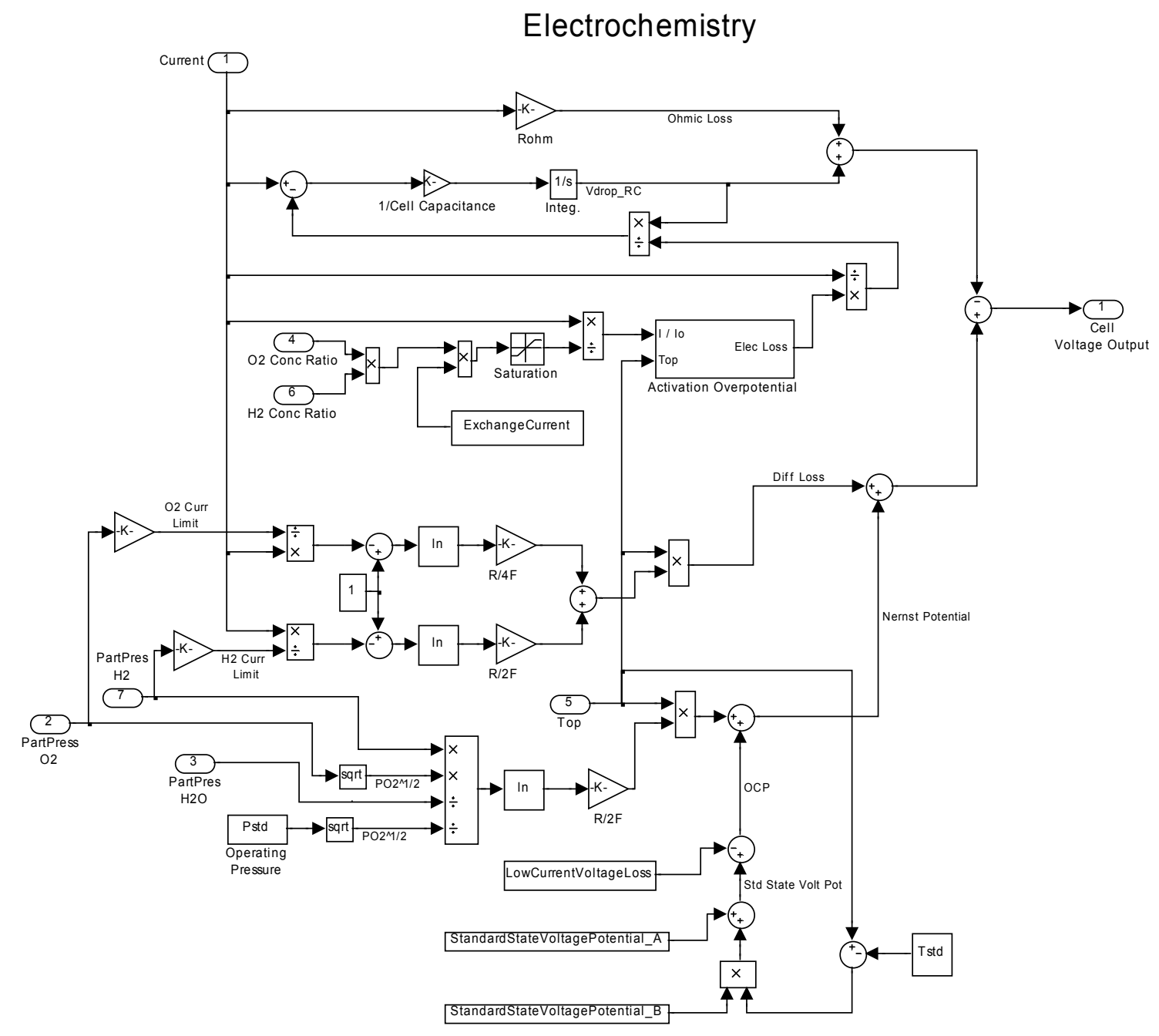

Figure 3.8: Electrochemistry Block Diagram 


\section{Activation Overpotential}

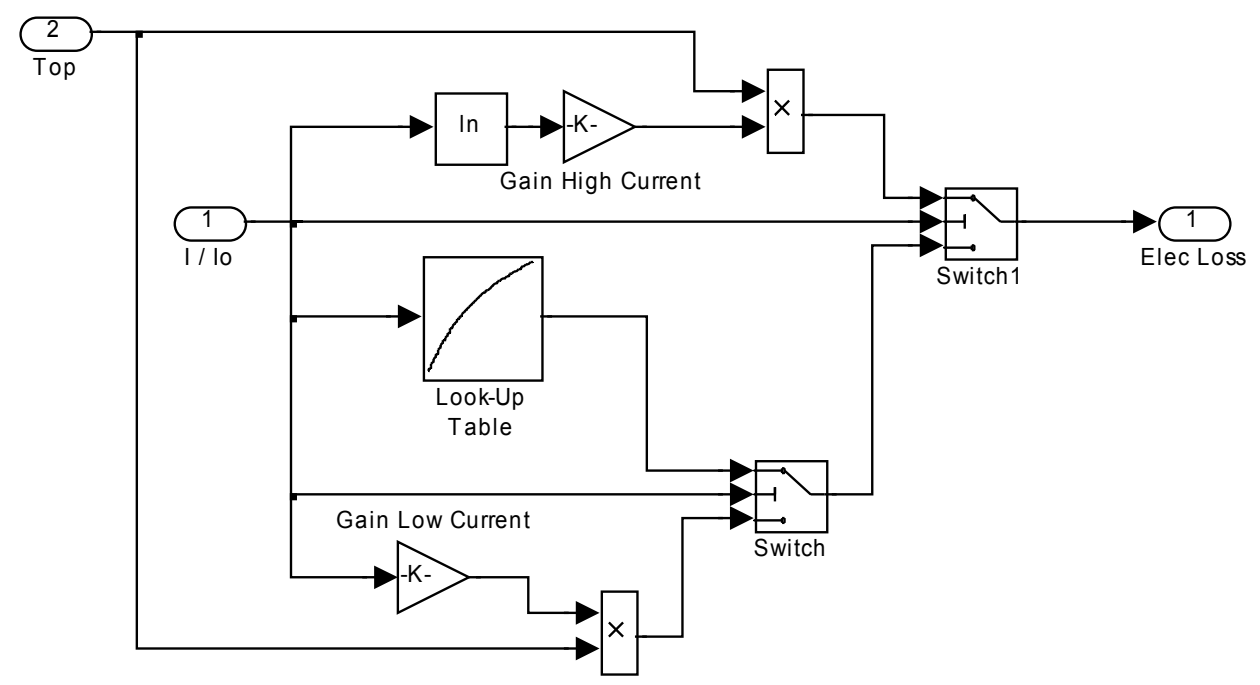

Figure 3.9: Activation Overpotential Calculation Block Diagram

\section{Hydrogen Diffusion}

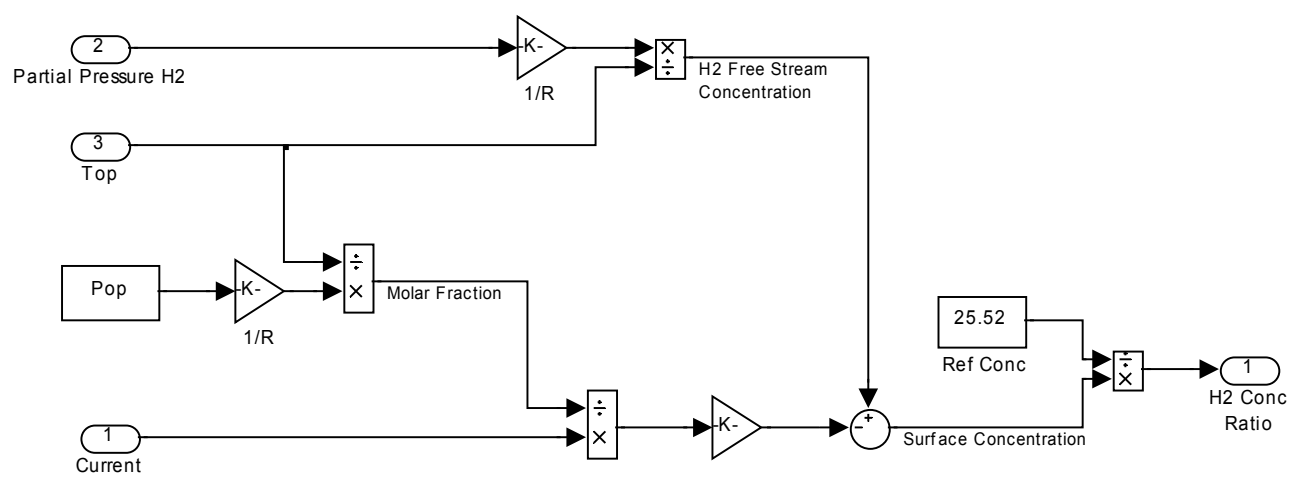

Figure 3.10: Anode $\mathrm{H}_{2}$ Diffusion Gradient Block Diagram

After performing all the above calculations, the stack voltage is determined by summing the four individual cell voltages, as illustrated in Figure 3.2.

The model operated in two modes for this work. The first is where the DC current gradually ramped up from $0 \mathrm{~A}$ to the current limit to generate a VI Curve. The second mode was a constant DC current which was used to simulate the EIS response. The System Load block of Figure 3.1 is shown in detail in Figure 3.11. The mode was 
set by controlling the position of the switches in the right of the diagram at run time. Switch 1 was always set in the down position to remove the Load Resistance parameter, which was not used for this work. Switch 2 then determined the mode used. The up position is the current ramp mode, while the down position is the constant current mode.

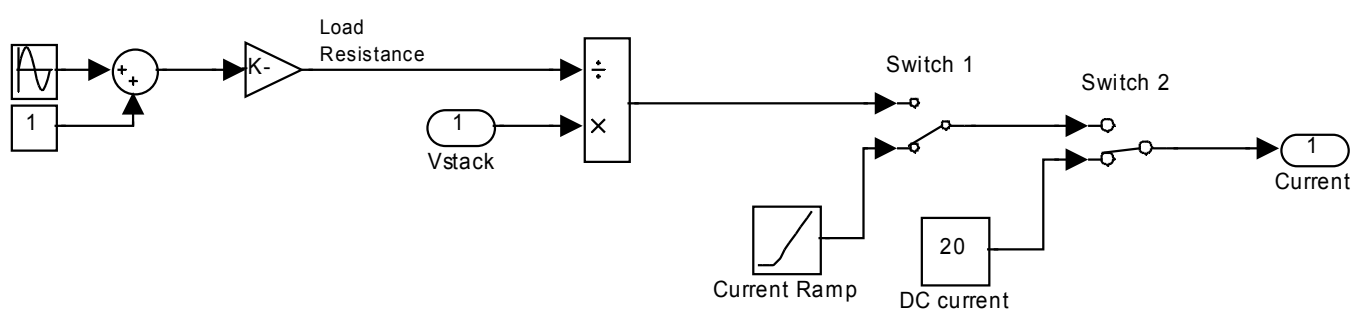

Figure 3.11: System Load Block Diagram

\subsubsection{Model Equations}

This section describes the equations used by the model to determine the cell's operating behavior. The cell potential is given by,

$$
E=E_{N}-i r_{\text {ohmic }}-\eta_{a c t}-\eta_{\text {diff }}^{a}-\eta_{\text {diff }}^{c}
$$

The Nernst equation describes the ideal potential,

$$
E_{N}=E_{O}+\frac{R T}{Z^{\prime} F} \ln \left(\frac{p_{H_{2}} p_{O_{2}}^{1 / 2}}{p_{H_{2} O} p_{o}^{1 / 2}}\right)
$$

where $E_{o}$ is the standard state potential, $p_{o}$ is the reference standard state pressure, $F$ is the Faraday constant $\left(96,439\right.$ Coulomb/gm-mol) and $Z^{\prime}$ is the number of electrons involved in the reaction. The electrochemical activation overpotential, $\eta_{a c t}$, is found by solving numerically the Butler-Volmer equation,

$$
i=i_{o}\left[\exp \left(\frac{\alpha Z^{\prime} F}{R T} \eta_{a c t}\right)-\exp \left(\frac{(\alpha-1) Z^{\prime} F}{R T} \eta_{a c t}\right)\right]
$$


where $i_{o}$ is the exchange current density. In this bulk model, the exchange current density is given by a variation of the equation used by Bernardi, et al. [49] to include the hydrogen concentration in the anode and the oxygen concentration in the cathode,

$$
i_{o}=i_{o}^{r e f}\left(\frac{C_{H_{2}}}{C_{H_{2}}^{r e f}}\right)\left(\frac{C_{O_{2}}}{C_{O_{2}}^{r e f}}\right)
$$

The original equation used by Bernardi, et al. only dealt with the cathode reaction and therefore, related the proton $\left(\mathrm{H}^{+}\right)$concentration that migrated through the membrane and the oxygen concentration to the exchange current density. For this work, the equation has been generalized to include both anode and cathode reactions by replacing the proton concentration with the hydrogen $\left(\mathrm{H}_{2}\right)$ concentration. For the bulk model, this is a valid substitution since the proton concentration would directly follow the hydrogen concentration. In this equation, $C_{H_{2}}$ is the hydrogen concentration at the anode gas diffusion electrode/catalyst layer interface and $\mathrm{C}_{\mathrm{H}_{2}}^{\text {ref }}$ is the reference hydrogen concentration at no load conditions. At the operating conditions stated, the reference hydrogen concentration is $25.52 \mathrm{~mol} / \mathrm{m}^{3}$. Likewise, $C_{O_{2}}$ is the oxygen concentration at the cathode gas diffusion electrode/catalyst layer interface and $C_{O_{2}}^{\text {ref }}$ is the reference concentration, calculated to be $4.623 \mathrm{~mol} / \mathrm{m}^{3}$. The gas diffusion electrode/catalyst layer interface concentration for anode and cathode was calculated by solving the electrode diffusion equation (cf. Figure 3.10),

$$
i=\frac{\left(C_{j}^{f s}-C_{j}\right) D_{j}^{\prime} Z^{\prime} F}{\delta} \cdot \frac{p_{j}}{R T}
$$

where $C_{j}^{f s}$ is the free stream concentration of reactant $\mathrm{j}$ and $\mathrm{p}_{\mathrm{j}}$ is the partial pressure of reactant $\mathrm{j}$, where $\mathrm{j}$ is $\mathrm{O}_{2}$ for the cathode and $\mathrm{H}_{2}$ for the anode. $D_{j}^{\prime}$ is the effective diffusion coefficient of reactant $j$, given by, 


$$
D_{j}^{\prime}=D_{j}\left(\frac{T}{T_{r e f}}\right)^{3 / 2}\left(\frac{p_{j}^{r e f}}{p_{j}}\right)\left(\frac{\varepsilon}{\tau}\right)
$$

The diffusion overpotential is given by,

$$
\eta_{\operatorname{diff}(j)}=-\frac{R T}{Z^{\prime} F} \ln \left(1-\frac{i}{i_{\operatorname{diff}(j)}}\right)
$$

where $i_{\text {diff(j) }}$ is the diffusion current limit of reactant $j$. For this simplified model, the diffusion current limit, $i_{\text {diff(j) }}$, is given by

$$
i_{\text {diff }(j)}=\frac{Z^{\prime} F D_{j}^{\prime}}{\delta} \cdot \frac{p_{j}}{R T}
$$

where $p_{j}$ is the partial pressure of reactant $\mathrm{j}$.

\subsubsection{Model Parameter Values}

The parameter $i_{o}^{r e f}$ was determined by fitting the polarization curve of the model to curves taken from the fuel cell in the lab. Values for the total ohmic resistance, $r_{\text {ohmic, }}$ and the cell double layer capacitance, $\mathrm{C}_{\mathrm{DL}}$, were based on lab measurements using the Electrochemical Impedance Spectroscopy technique on the four cell PEM stack. The high frequency resistance value was used for $r_{\text {ohmic }}$. The cell capacitance was determined from an equivalent circuit fit to the measured data. Another term, Low Current Voltage Loss, was added to correct the model's Open Circuit Voltage to the experimental value. The values of model parameters are given in Table 3.1. 
Table 3.1: Model Parameters

\begin{tabular}{|c|c|c|c|}
\hline Variable & Name & Value & Reference \\
\hline $\mathrm{A}_{\text {cell }}$ & Cell Active Area $\left[\mathrm{m}^{2}\right]$ & 0.01337 & Experiment \\
\hline $\mathrm{p}$ & Operating Pressure [Pa] & 101000 & Experiment \\
\hline $\mathrm{T}$ & Operating Temperature $[\mathrm{K}]$ & 343 & Experiment \\
\hline $\mathrm{E}_{\mathrm{o}}$ & Standard State Potential [Volts] & 1.19 & Assumed \\
\hline $\mathrm{r}_{\text {ohmic }}$ & Cell Resistance $\left[\mathrm{ohm}-\mathrm{cm}^{2}\right]$ & 0.2408 & $\begin{array}{l}\text { VI Curve / } \\
\text { EIS }\end{array}$ \\
\hline $\mathrm{C}_{\mathrm{DL}}$ & Double Layer Capacitance [F/cm²] & 0.0357 & EIS \\
\hline$i_{o}^{r e f}$ & Exchange Current Density $\left[\mathrm{Amp} / \mathrm{cm}^{2}\right]$ & 0.0017 & VI Curve \\
\hline-- & Low Current Voltage Loss [Volts] & 0.2528 & VI Curve \\
\hline$\alpha$ & Transfer Coefficient & 0.5 & Gemmen [48] \\
\hline$\tau$ & Diffusion Layer Tortuosity & 4 & VI Curve \\
\hline$\varepsilon$ & Diffusion Layer Porosity & 0.4 & Gemmen [48] \\
\hline$\delta$ & Diffusion Layer Thickness [m] & 0.0004 & Experiment \\
\hline$D_{H_{2}}$ & Hydrogen Diffusion Coefficient $\left[\mathrm{m}^{2} / \mathrm{sec}\right]$ & $1.1 \mathrm{e}-4$ & Assumed \\
\hline$D_{\mathrm{O}_{2}}$ & Oxygen Diffusion Coefficient $\left[\mathrm{m}^{2} / \mathrm{sec}\right]$ & $2.84 \mathrm{e}-5$ & Wang [50] \\
\hline
\end{tabular}

\subsection{Model Setup}

This section describes the overall setup procedure and how the model was setup in order to perform both VI curve and EIS response measurements.

\subsubsection{Model Execution}

The MATLAB model consists of two files, a setup file (.m file extension) and the pictorial Simulink block diagram file (.mdl file extension). The setup file must be run before the model can execute to declare variables that are used in the model and set default values. The file also performs some calculations that can be performed before model execution to save CPU time. In addition, the setup file can be used to set the initial conditions of the model. The setup file is executed from the MATLAB command window. After executing, the MATLAB workspace is populated with the variables used in the model. Once this is done, the PEMFC model can be run in the Simulink environment. The code listing for the setup file is included in the Appendix. 


\subsubsection{Curve Setup}

The VI curve setup was implemented by switching the current mode to the ramp function (cf. Figure 3.11). The purpose of the VI curve is to characterize the stack voltage for all possible currents. This ranges from no current (at open circuit) up to the maximum current. Since the model cannot operate at exactly $0 \mathrm{~A}$, a value of $1 \mathrm{~mA}$ was used to approximate open circuit conditions. Therefore, the ramp function is setup to start at $0.001 \mathrm{~A}$ and ramp the current continuously until the stack voltage falls to zero.

\subsubsection{VI Curve Initial Conditions}

As with any model, the initial conditions are very important. When calculating the VI curve, there are two ways of establishing the proper initial conditions. The first is to simply let the model run for a long period of time at a constant current load of $1 \mathrm{~mA}$. This can be done by setting the delay on the ramp function to a large value $(>100 \mathrm{~s})$, which waits until the time specified before starting to ramp the current. The other method is to initialize all the unknown parameters to the value they would have in the $1 \mathrm{~mA}$ state. In this way, the VI curve can start to be acquired immediately with very good accuracy.

\subsubsection{VI Curve Calculation Procedure}

The VI curve was measured with the model initialized to the $1 \mathrm{~mA}$ steady state. This is done by entering the initial values into the setup file so that when it runs it establishes steady state conditions. Once the setup file has been executed, ensure that Switch 1 in the System Load block is set to the down position and Switch 2 is set to the up position to engage the ramp function. To run the model, click on the 'Start Simulation' button along the toolbar in Simulink, shown in Figure 3.12. 


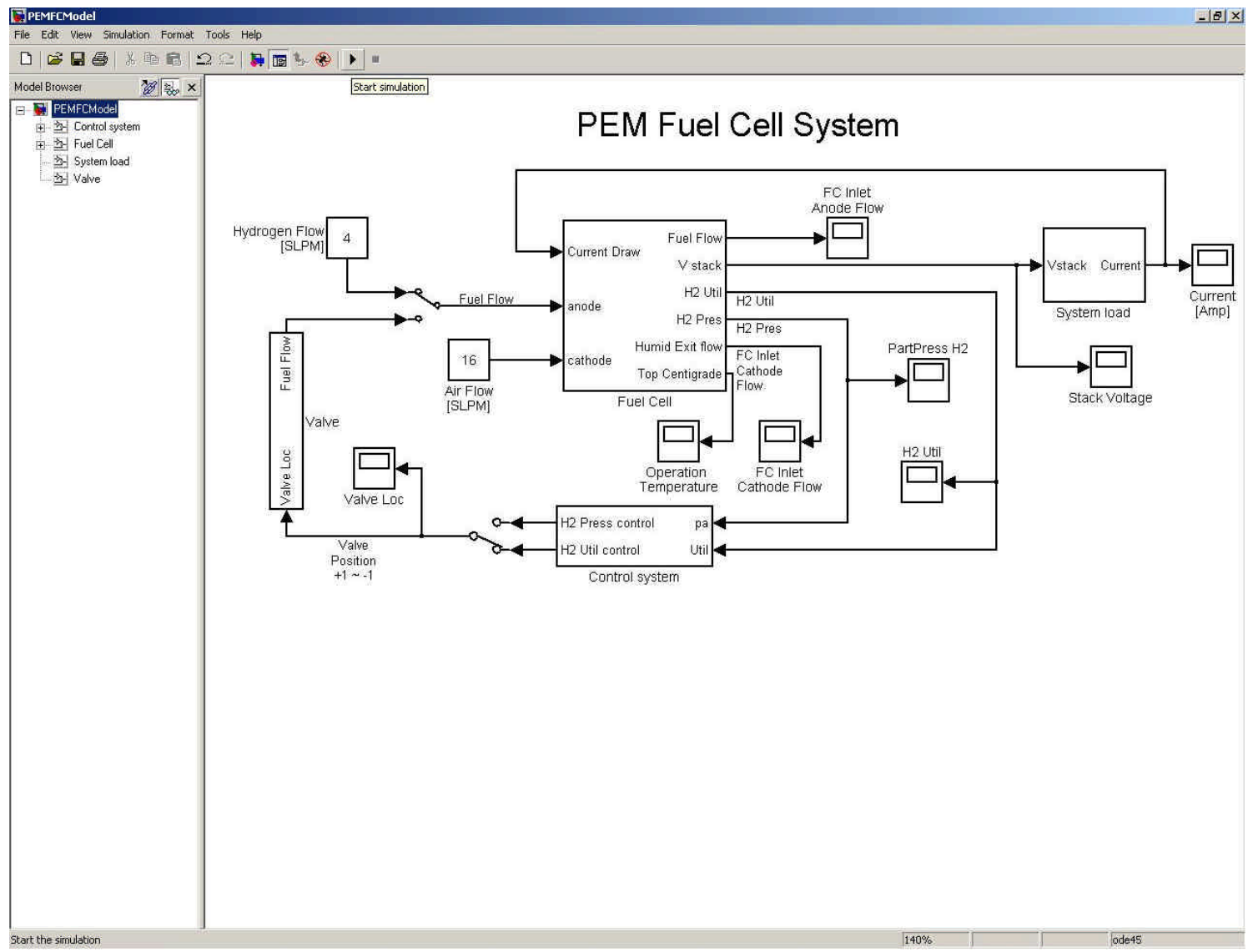

Figure 3.12: Run the Model to Generate a VI Curve

\subsubsection{EIS Setup}

The EIS response of the PEMFC stack model was accomplished using the Linear Analysis tool that is part of the Control System Toolbox, v.5.0. This section will describe the setup required to use the Linear Analysis tool to measure an EIS response of the stack model. The procedure used to measure the response will also be outlined.

\subsubsection{EIS Initial Conditions}

Before an EIS measurement can be simulated, the model needs to be in steadystate operation. Since the Linear Analysis tool can not be used when the model is running, the only way to acquire steady-state operation is to adjust the initial conditions. The Linear Analysis tool has this functionality built in. However, due to an apparent software bug, or the complexity of the PEMFC stack model, the function would not correctly adjust the initial conditions. To remedy this, each block in the model that required an initial condition had to be initialized in the setup file before using the Linear 
Analysis tool. Each block in the model that required an initial condition, was given a variable as the initial condition. That variable is then set to the appropriate value when the setup file executes.

The only other parameter that requires an initial value is the DC current. This is set at run time in the System Load (cf. Figure 3.11) block by changing the value of the DC Current constant input. This value should match the current value used to determine the initial conditions the last time the setup file was executed.

\subsubsection{EIS Calculation Procedure}

The Linear Analysis tool measures the frequency response of the model and can display the information in a variety of plots, including Nyquist (Complex Plane) plots and Bode plots. To simulate a response with the Linear Analysis tool, first open the tool by selecting the "Linear Analysis" item from the "Tools" menu in Simulink as shown in Figure 3.13.

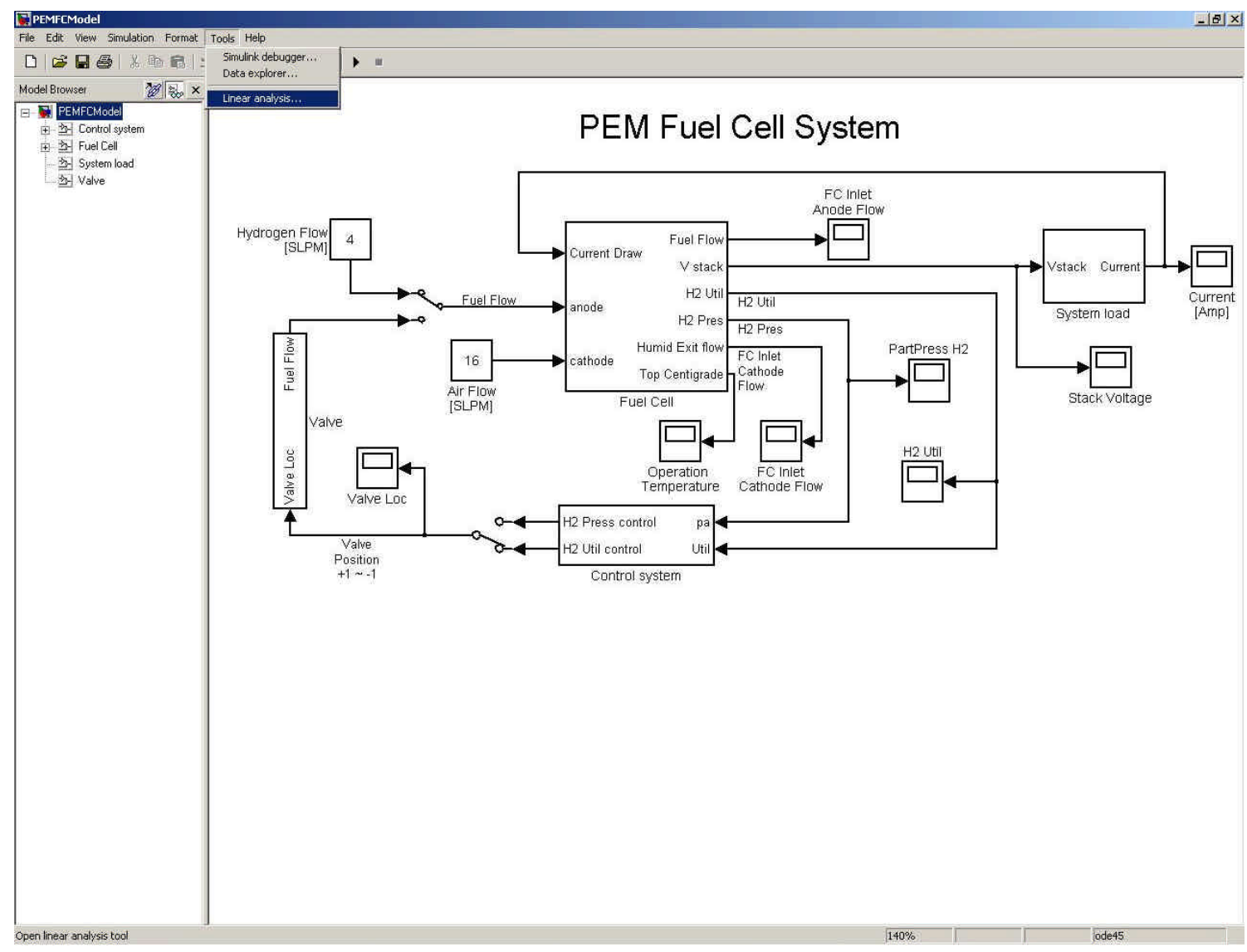

Figure 3.13: Open Linear Analysis Tool 
The Linear Analysis tool will open two windows as depicted in Figure 3.14. The main window is the LTI Viewer, which will display the plots of the simulated responses. The other window contains input and output points. These points can be dragged and dropped with the mouse and placed on the model. The simulated response will be determined by the placement of the points. The response is the effect generated at the output point by a small signal applied at the input point.

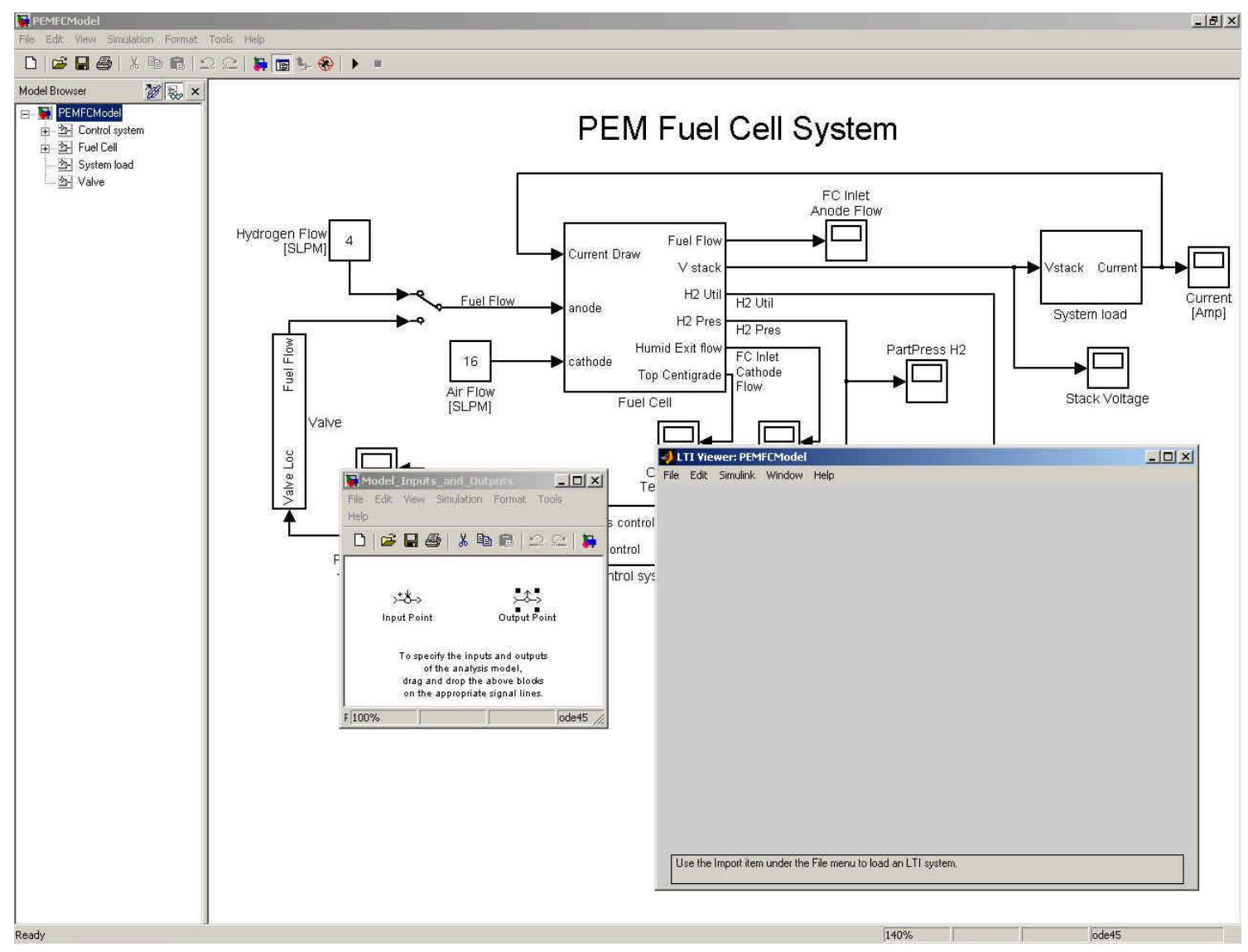

Figure 3.14: Linear Analysis Tool

Therefore, to simulate a stack response, drag the Input point to the current line on the model and drag the Output point to the stack voltage line. Both points will be added to the model automatically. This is shown in Figure 3.15, with the points placed in the upper right corner of the picture. 


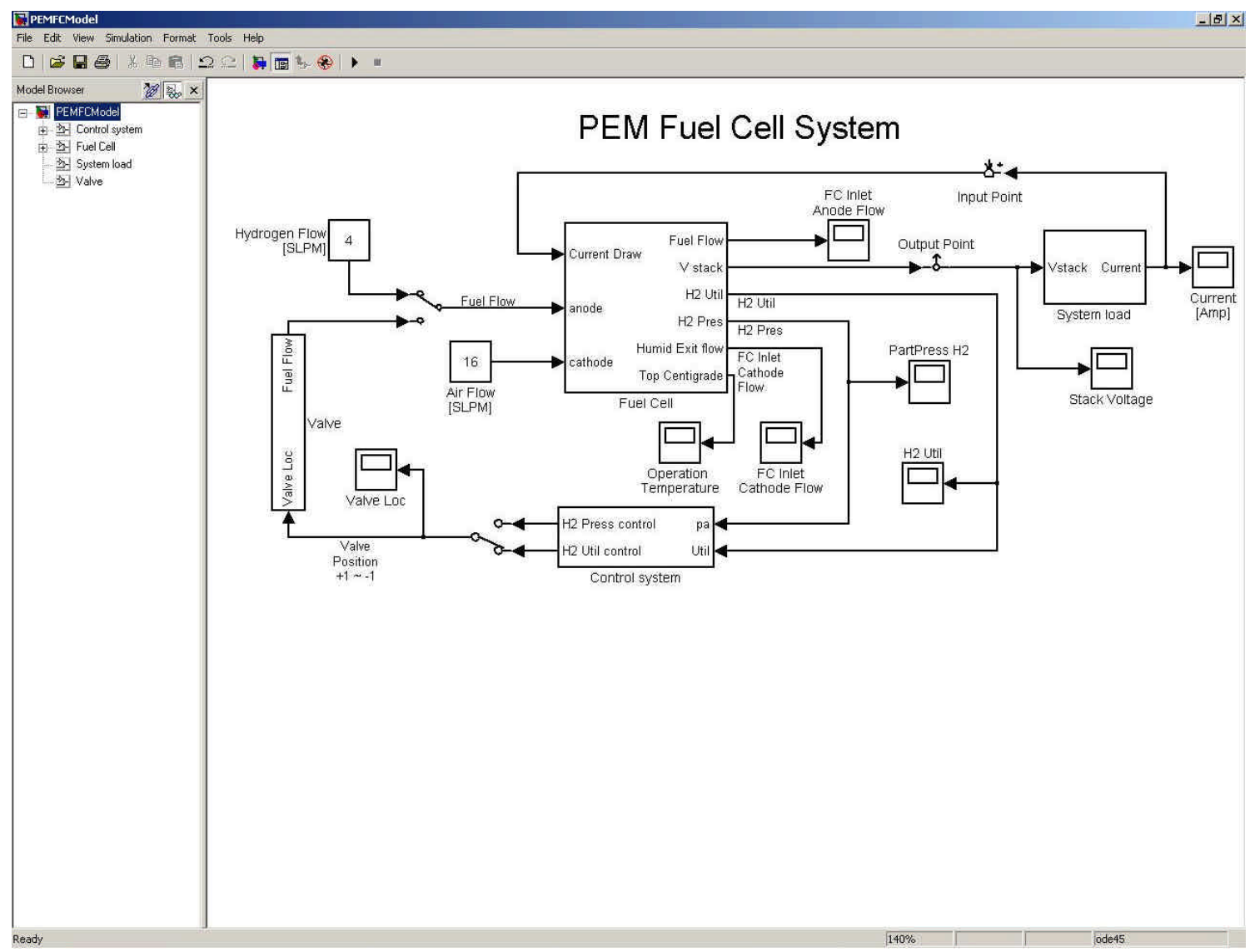

Figure 3.15: Placing Input and Output Points

Then, the LTI Viewer is used to simulate the response. The first step is selecting the type of plot to display the response, usually Nyquist or Bode. This can also be changed after a response has been generated. To choose the type of plot, select the 'Plot Configurations' item on the 'Edit' menu of the LTI Viewer as shown in Figure 3.16. This will open a separate window where the number of plots and the type of plots can be selected, seen in Figure 3.17. 


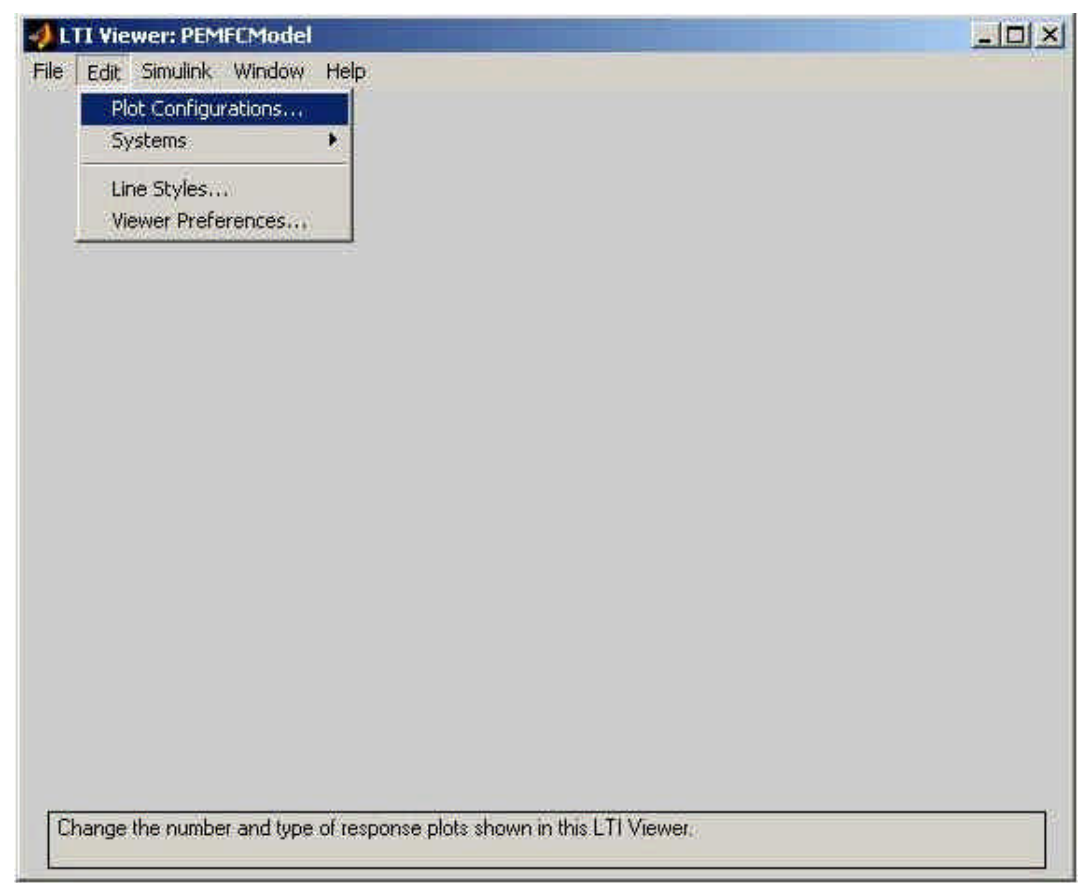

Figure 3.16: Select Plot Configuration

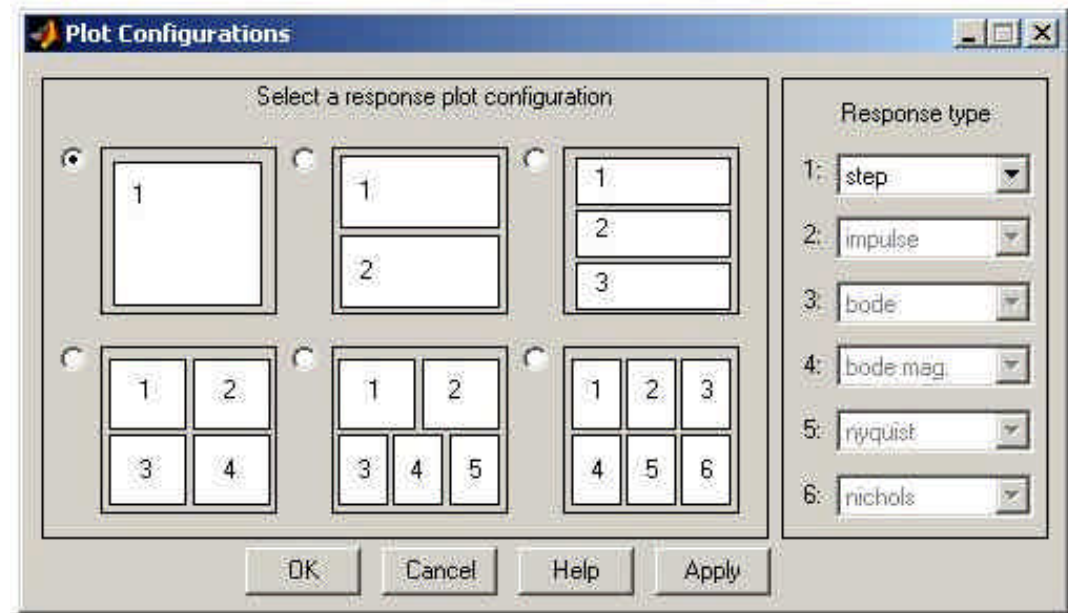

Figure 3.17: Plot Configuration Window

The number of plots to display on the LTI Viewer can be chosen by selecting from the various configurations shown. To choose the type of plot displayed, select from the drop-down box on the right that matches the appropriate plot. Figure 3.18 shows a Nyquist plot being selected from the drop-down list. 


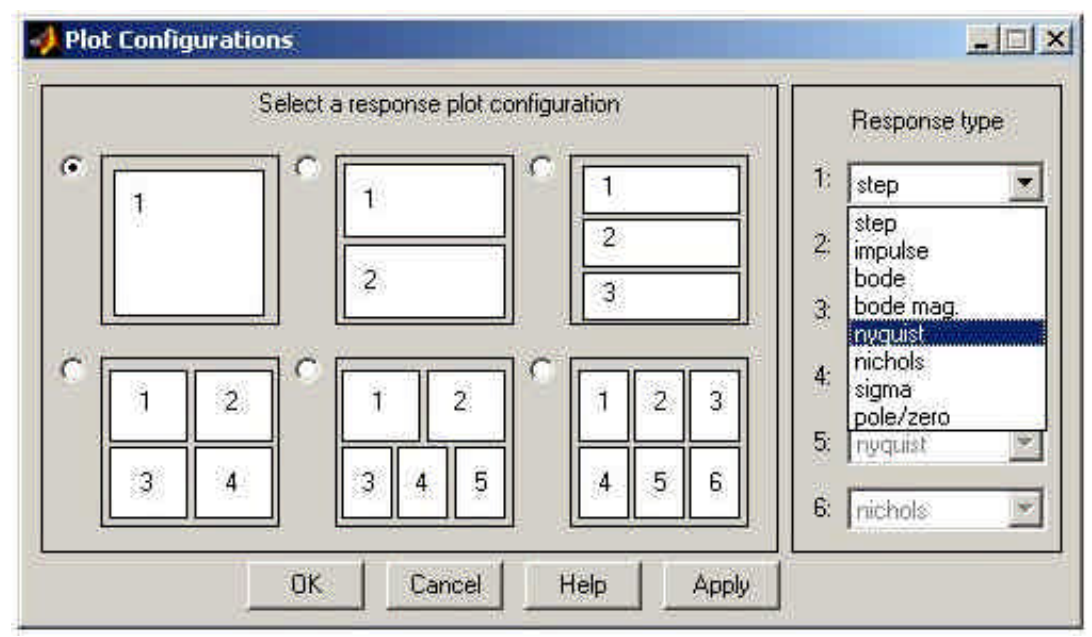

Figure 3.18: Select Nyquist Plot

To acquire a response, select the 'Get Linearized Model' item from the 'Simulink' menu on the LTI Viewer as shown in Figure 3.19.

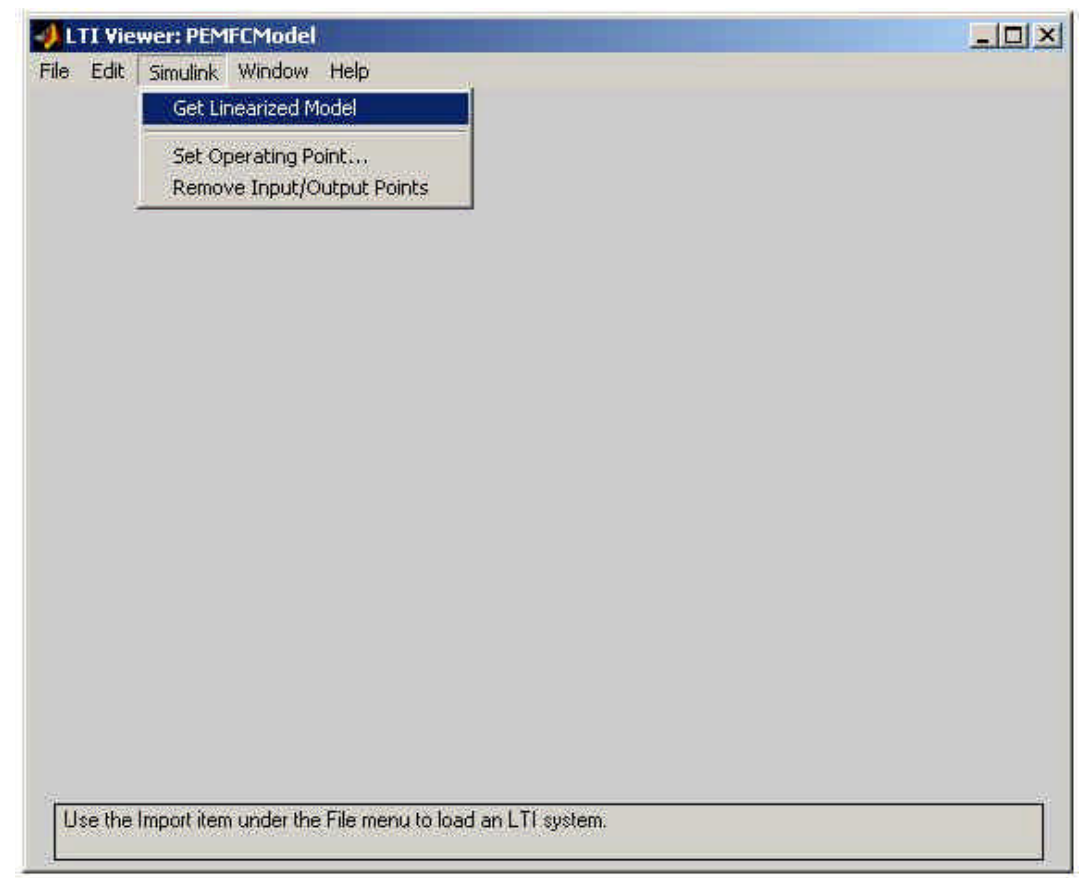

Figure 3.19: Simulate Response using LTI Viewer

\subsubsection{Scaling the Model EIS Response}

After a model EIS response has been generated, the LTI Viewer will display the plot. An example plot is shown in Figure 3.20. This plot is the raw signal as calculated by Simulink when performing a stack response at a DC current load of $20 \mathrm{~A}$. 


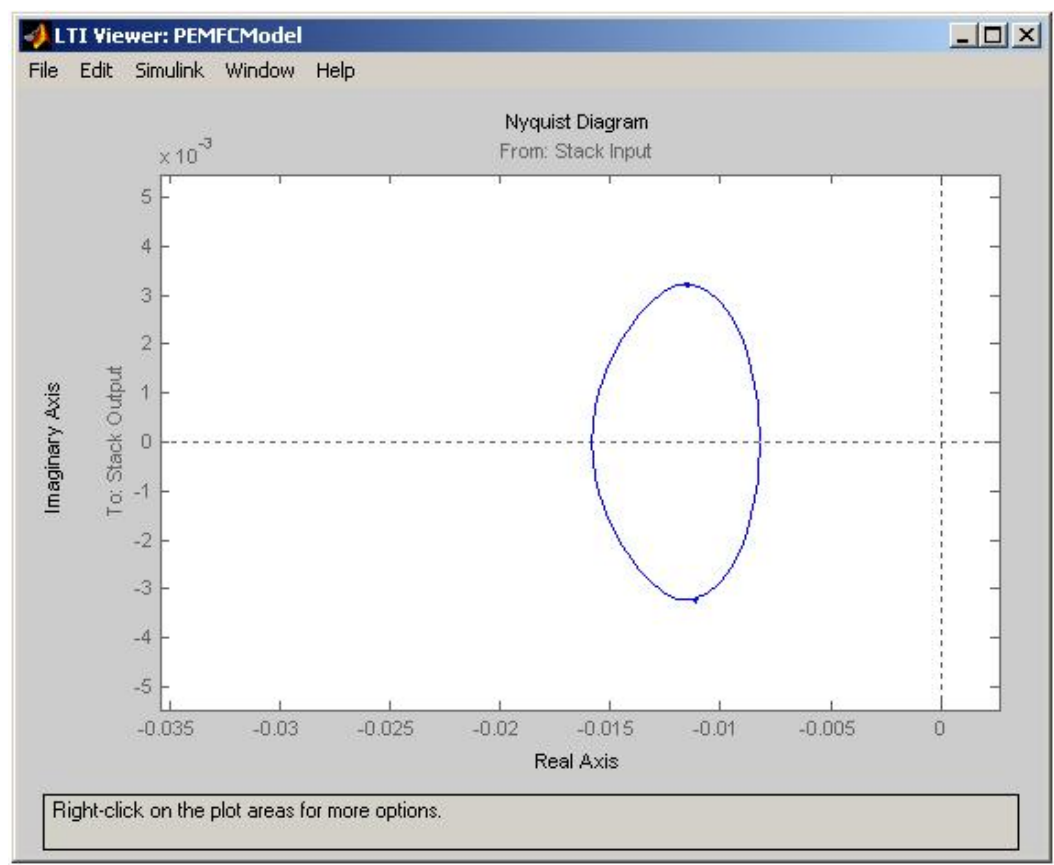

Figure 3.20: Raw Nyquist Response for Stack at 20A

There are several observations to make about the plotted response. First, the Linear Analysis tool calculates the response for both positive and negative frequencies. This is why the response is a closed-loop shape. Also, the entire response is to the left of the Imaginary Axis, meaning the real portion of the response is negative. This is due to the procedure used to calculate the cell voltage (cf. Figure 3.8). Specifically, the model calculates the losses and subtracts them from the ideal cell potential. These losses are variable, whereas the ideal cell potential is a constant (for a given operating condition). When the EIS response of the model is found, it is the loss mechanisms which are generating the response. The response of the ideal cell potential, a constant, is zero - the ideal cell potential will not alter the applied signal. Thus, when the cell potential is found by taking the ideal potential and subtracting the losses, the response becomes negative because a positive response (from the sum of the losses) is being subtracted from a zero response (the ideal cell potential). Therefore, the model's response should be multiplied by a negative one to change the sign back to positive. Lastly, the magnitude of the response is quite small because it is the total response and has not yet been normalized to account for cell area. Before the model response can be compared to the experimental response, the model response needs to be scaled to remove all these effects. 
The process for correcting the raw response is simple. From the LTI Viewer, select the 'Export' item on the 'File' menu. A dialog box will appear showing all available responses. Highlight the responses from the list and click the 'Export to Workspace' button. This will save the responses as state-space models in the MATLAB workspace.

The raw response data was corrected in MATLAB using the M-file 'makezfile.m', which is listed in the Appendix. This file takes the state-space model and adjusts the response data by removing the negative frequency information, multiplying by a factor of -1 , and normalizes for area. The resulting response is plotted in a graph and also saved to a data file that can be read into ZView. The properly scaled response of Figure 3.20 is shown in Figure 3.21.

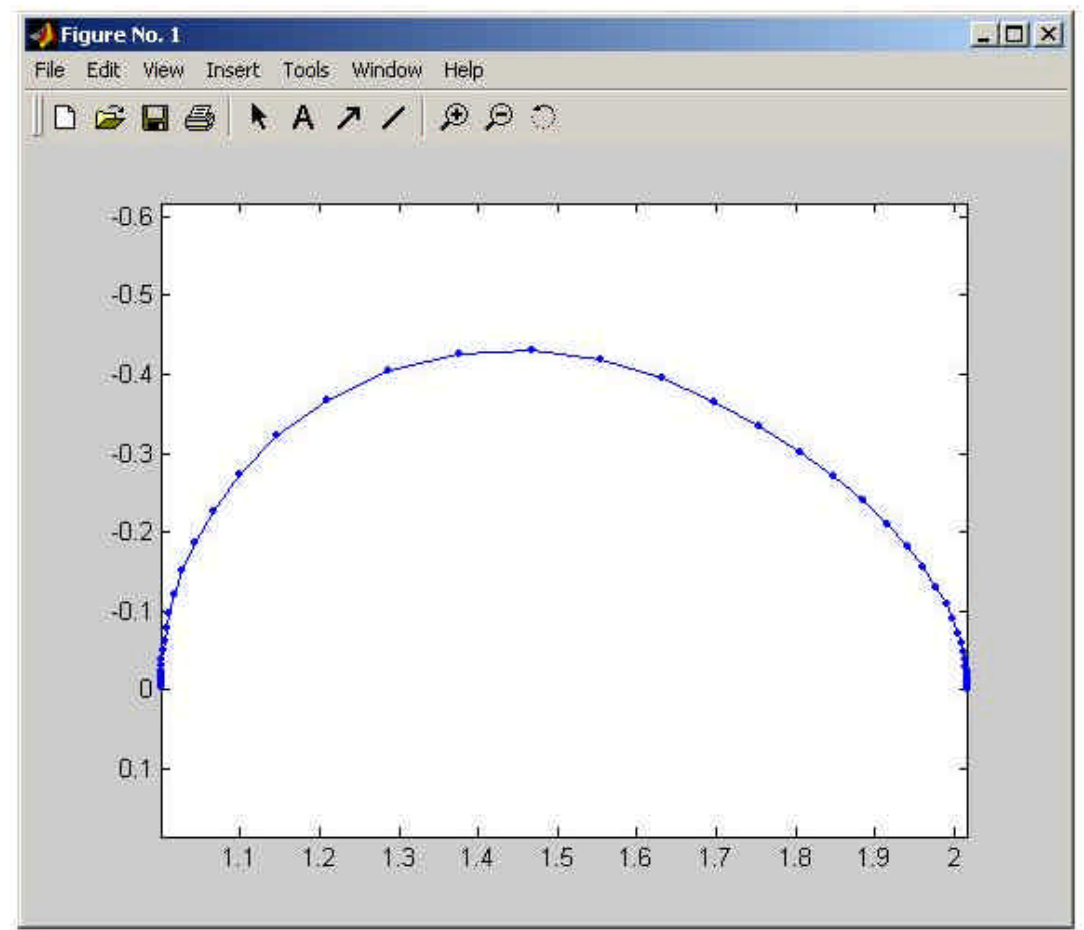

Figure 3.21: Corrected 20A Stack Response

\subsection{Modeling Results}

For this work, the four cell stack model was operated without the use of the control system or a load. Instead, the load current was directly specified. A ramp function provided the load current for the fuel cell when collecting a VI curve. This 
provided the polarization behavior of the fuel cell up to the limiting current, accounting for losses due to electrochemical activation, ohmic, and diffusion. To simulate the model EIS data, the model was operated at a constant current.

\subsubsection{Curve}

The VI curve of the PEMFC stack model was simulated to show the overall behavior of the fuel cell. The current was ramped from $1 \mathrm{~mA}$ up to the limiting current at a rate of $1 \mathrm{~A}$ per second of simulation time. Several rates were simulated to determine if the rate of change of the current affected the VI curve. It was found that the rate did not significantly affect the VI curve. The VI curve produced by the model is displayed in Figure 3.22.

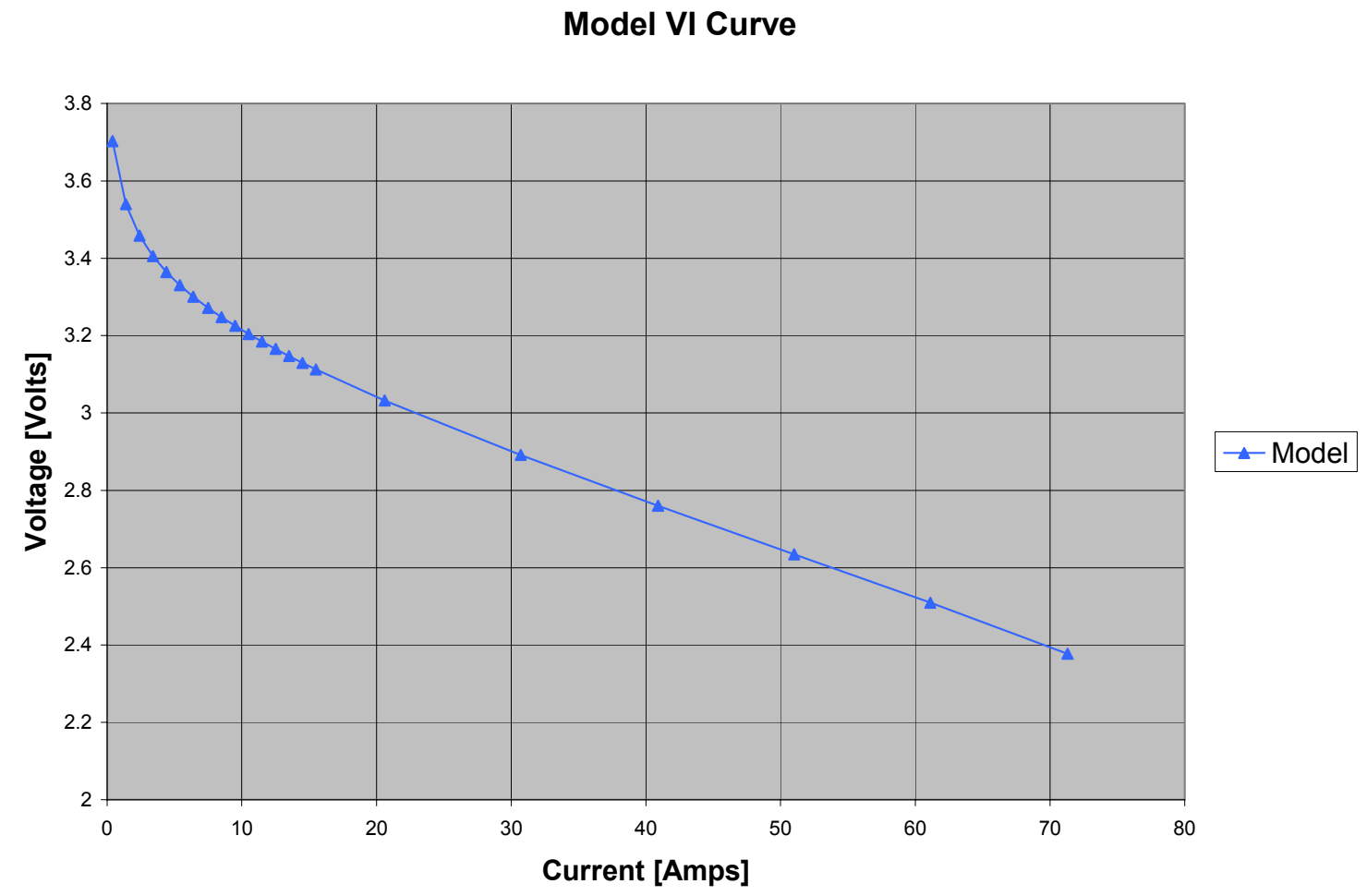

Figure 3.22: Model Simulated VI Curve

\subsubsection{EIS Response}

The model was used to predict the EIS responses while the PEMFC stack was operating at DC currents that were chosen to approximate the DC currents that were used when experimentally measuring the fuel cell EIS response. The DC current values used when simulating the response are listed in Table 3.2. 
Table 3.2: Comparison of DC currents

\begin{tabular}{|c|c|}
\hline Experimental DC Current Values [Amp] & Model DC Current Values [Amp] \\
\hline 0.2 & 0.2 \\
1 & 1 \\
1.8 & 1.8 \\
20 & 20 \\
35 & 35 \\
48 & 50 \\
59 & 60 \\
67 & 67 \\
\hline
\end{tabular}

The simulated EIS responses of the PEMFC stack are shown below in Figures 3.23 and 3.24. Figure 3.23 displays all the responses on the same plot, and Figure 3.24 is a close-up of the higher current simulations.

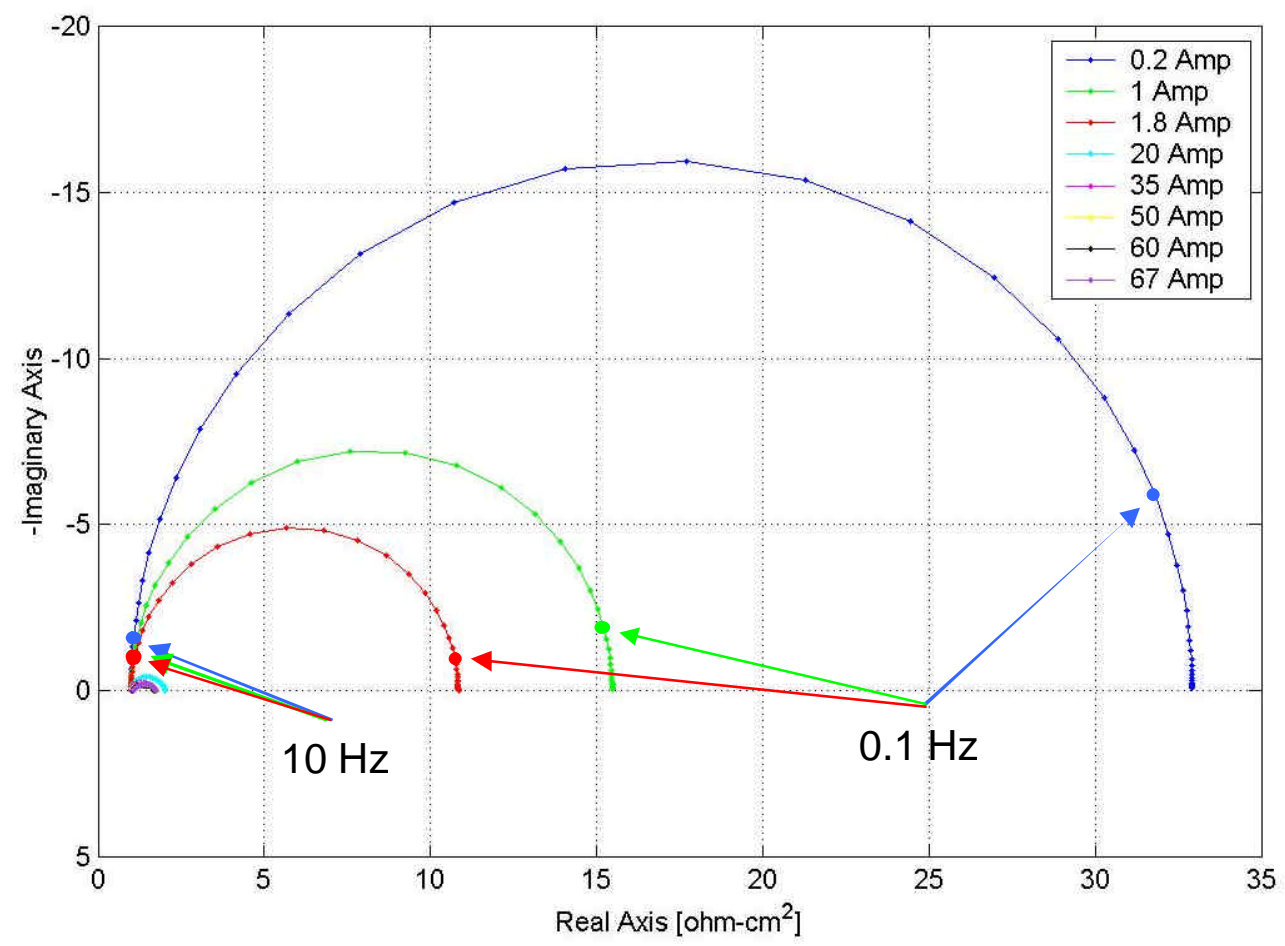

Figure 3.23: Simulated EIS Responses of the PEMFC Stack 


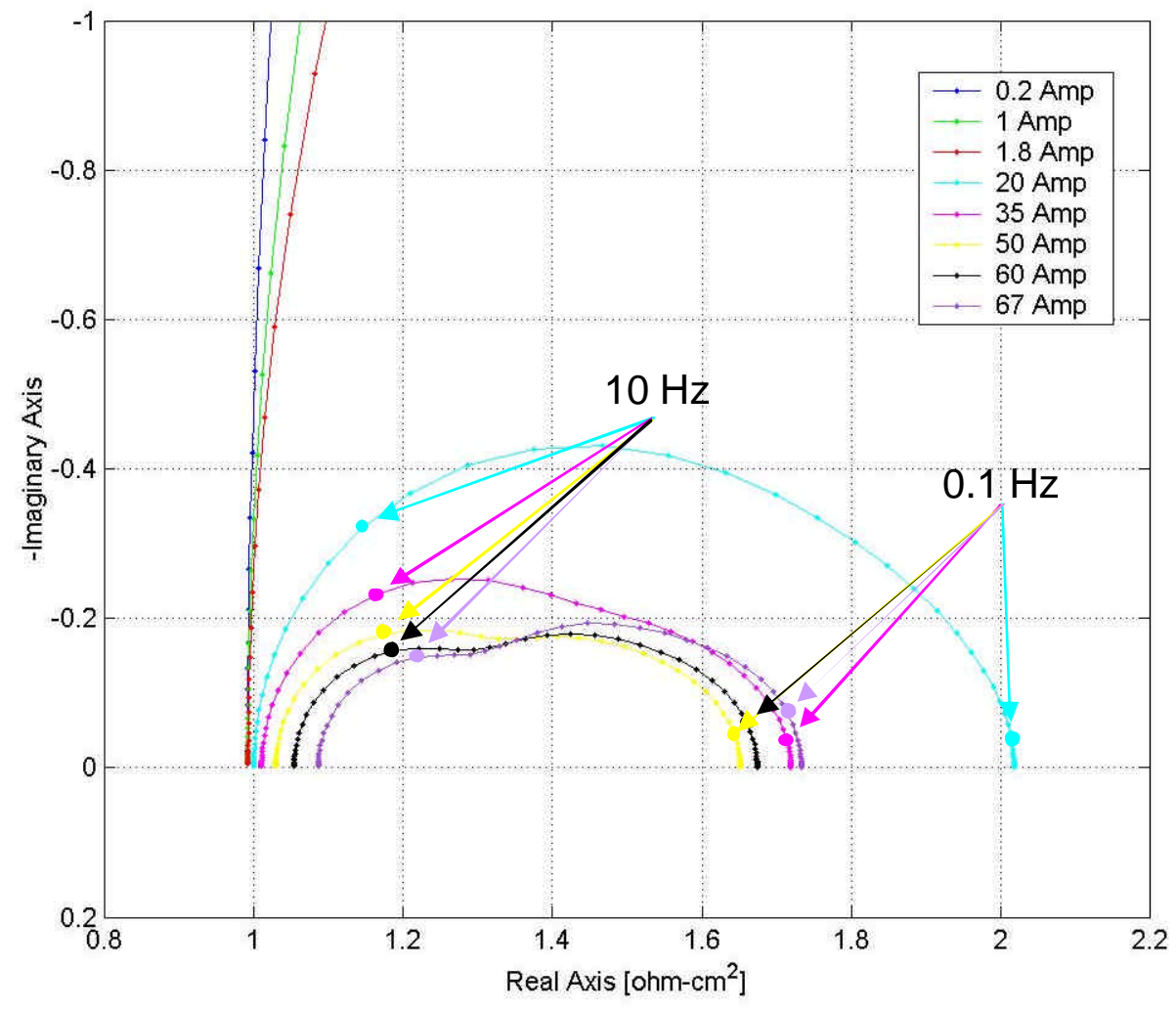

Figure 3.24: High Current Simulated EIS Responses of the PEMFC Stack

The following chapter will compare the results from the model simulation with the experimental results presented in Chapter Two. 


\section{Chapter 4: Discussion}

This chapter relates the experimental measurements of the four cell PEMFC stack with the simulated results generated by the MATLAB model. The VI curves and the EIS data are compared in the first two sections with a discussion in the last section.

\subsection{Comparison of Experimental and Model VI Curves}

The VI curve produced by the model was fitted to the experimental VI curve as a means of calibrating the model. The resulting VI curves are provided in Figure 4.1.

\section{Curve Comparison}

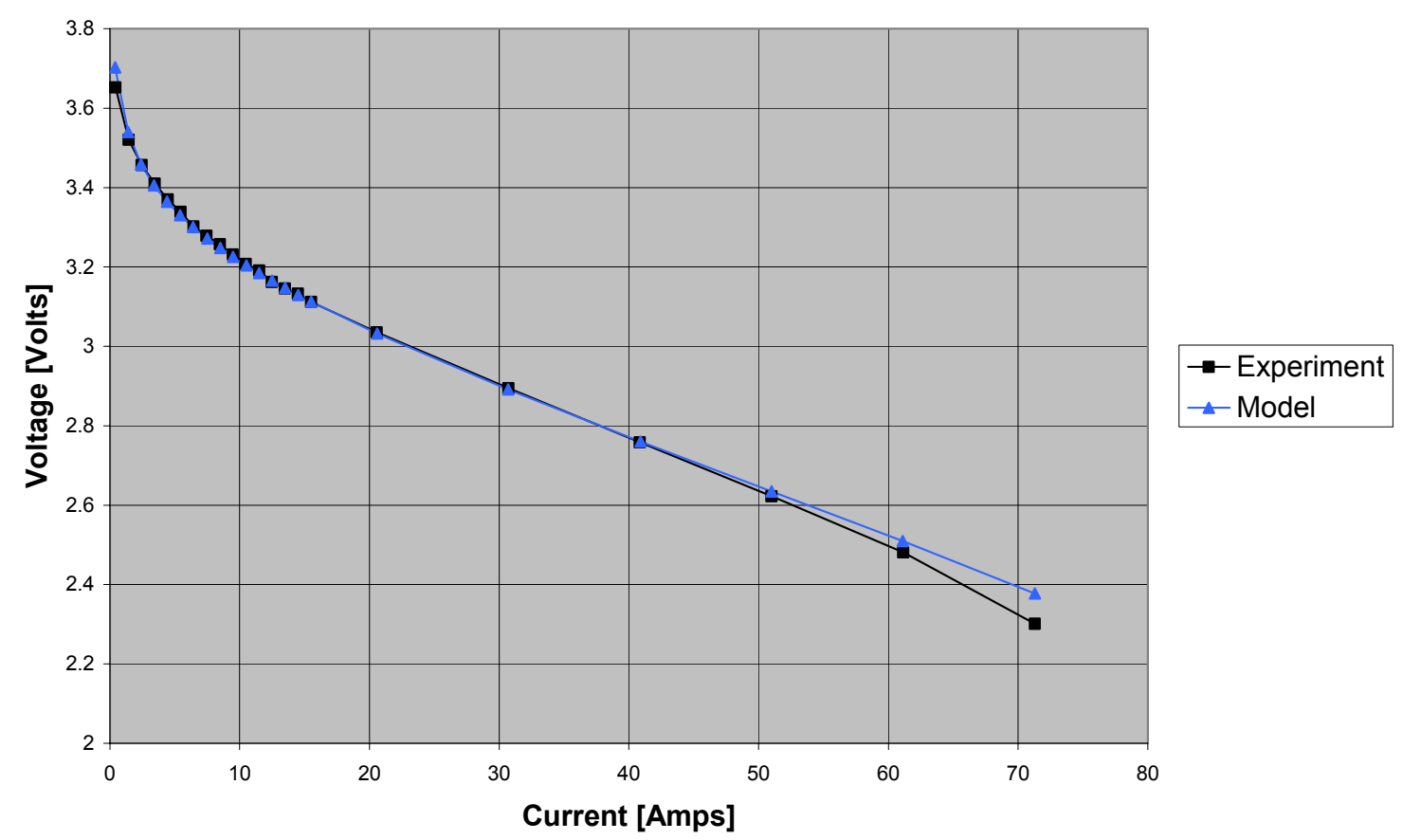

Figure 4.1: Comparison of Experimental and Model VI Curves

The model showed it could closely follow the experimentally measured stack VI curve for most of the current range examined in this work. The low current range was measured in one Amp increments to capture the curvature detail associated with the activation loss. The exchange current density, $i_{o}^{r e f}$, was adjusted until the model matched the curve, resulting in an exchange current density of $1.7 \mathrm{~mA} / \mathrm{cm}^{2}$. 
It can also be seen from the linear region that the ohmic losses of the model closely matched the experimental data. The high frequency resistance from the experimental EIS measurements showed a total stack ohmic resistance of $1 \Omega-\mathrm{cm}^{2}$. Assuming all four cells were the same, each cell had a resistance of $0.25 \Omega-\mathrm{cm}^{2}$. This value corresponds to the sum of all the resistive losses. The value used in the model is $0.2408 \Omega-\mathrm{cm}^{2}$, with the remainder of the difference from diffusion (cf. Figure 3.8).

\subsection{Comparison of Experimental Data and Model Simulations}

The results from both experimental measurements and modeling simulations of EIS are presented in this section. Each DC current case is presented individually, directly comparing the experimental data to the model simulation. The results from the $0.2 \mathrm{~A}$ measurement and simulation are shown in Figure 4.2.

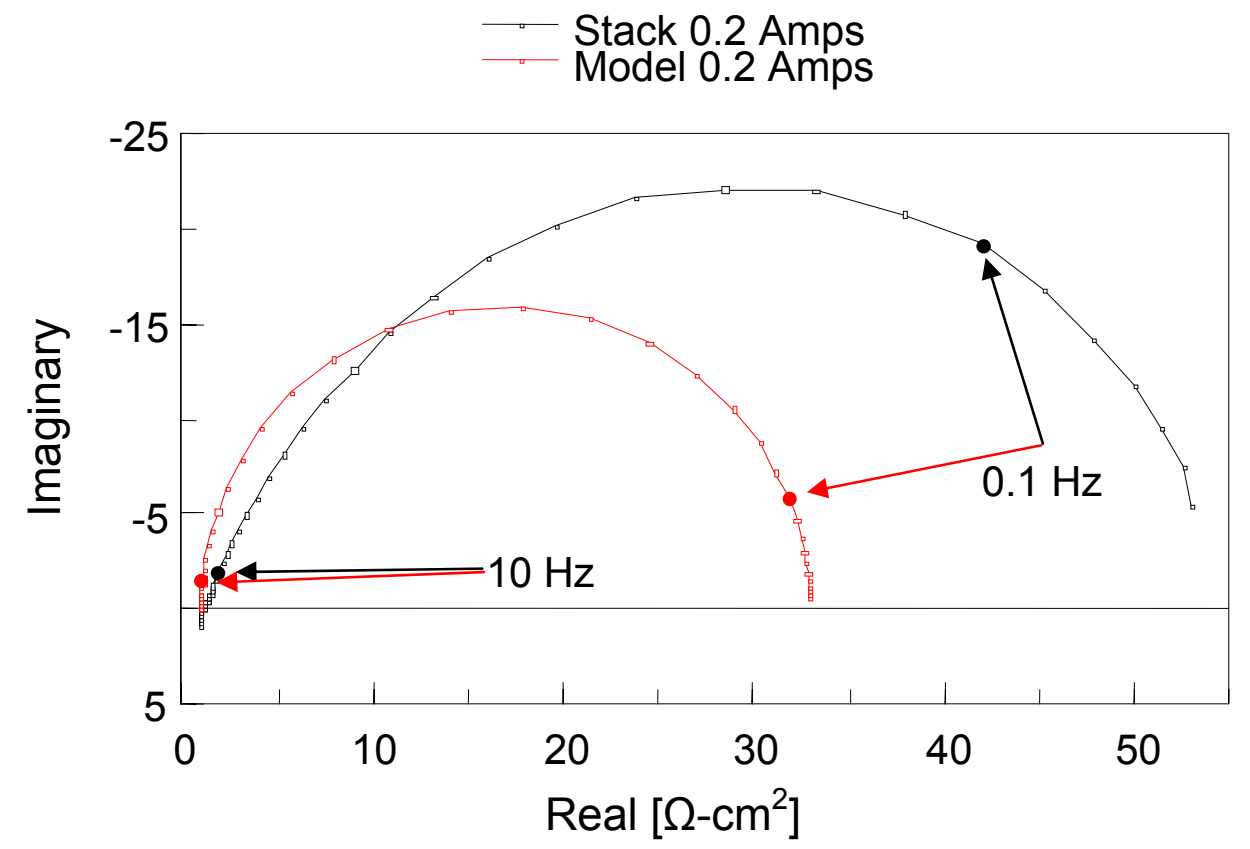

Figure 4.2: Comparison of Experimental Data and Model Simulation for Stack PEMFC at 0.2 Amps

It can be seen that, while the overall response is generally the same, the model does not closely fit the experimental data. The most noticeable difference is the model underestimates the magnitude of the response at the low frequency by almost a factor of two. However, if the data is plotted on a Bode plot, as in Figure 4.3, it can be seen that 
the model only significantly deviates from the experimental data when predicting the magnitude at the low frequencies (below $0.3 \mathrm{~Hz}$ ).

Another difference evident between the experiment and model is seen in the shape of the response curve. The model response is a true semi-circle, while the experimental curve is slightly depressed, meaning that the height is less than the radius of the curve. This is especially evident in the phase information of the Bode plot in the frequency range $100 \mathrm{~Hz}-0.1 \mathrm{~Hz}$.
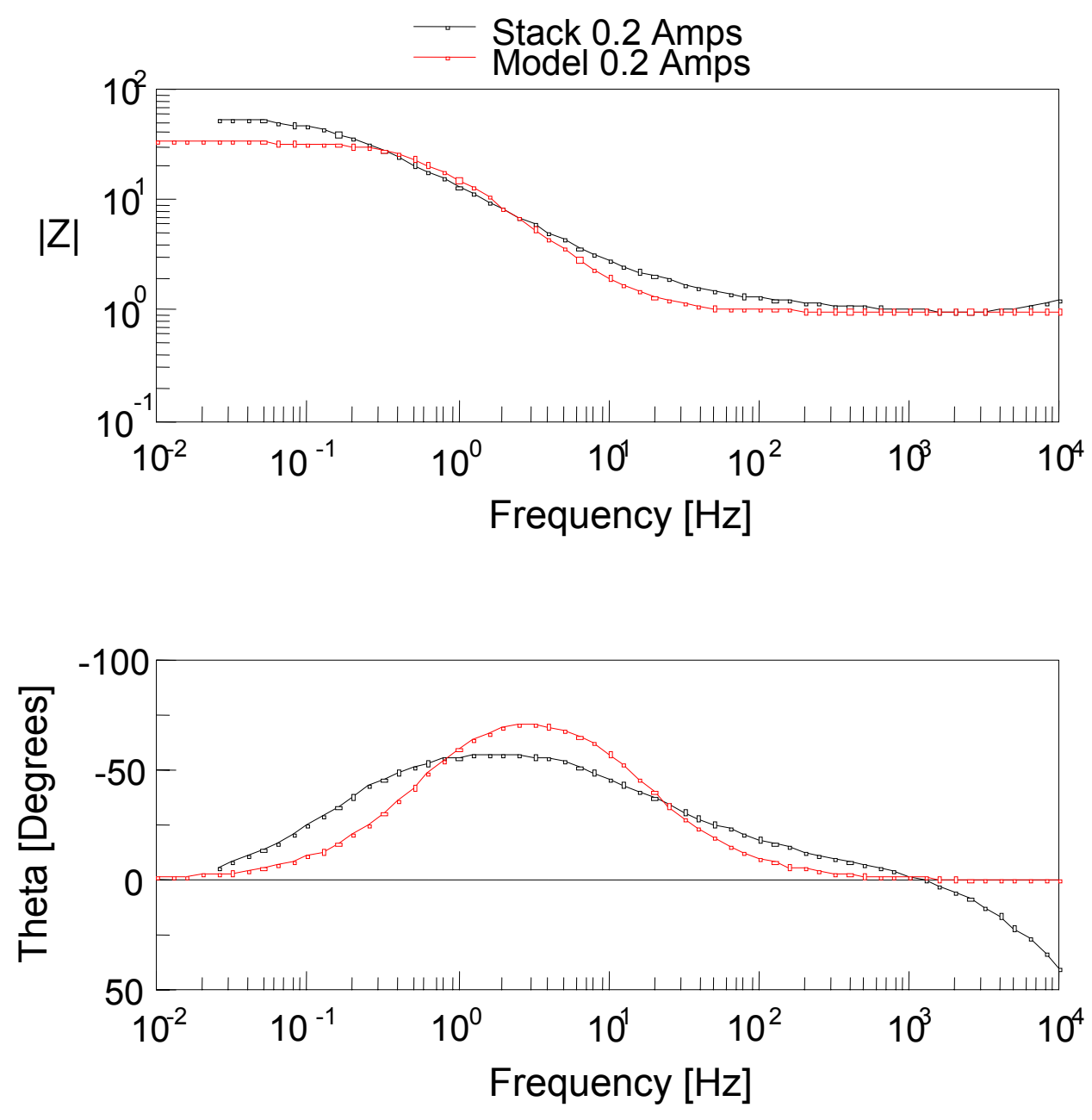

Figure 4.3: Bode Plot of 0.2A

The following series of figures compare the results of the other current settings in sequential order from low current to high current. 


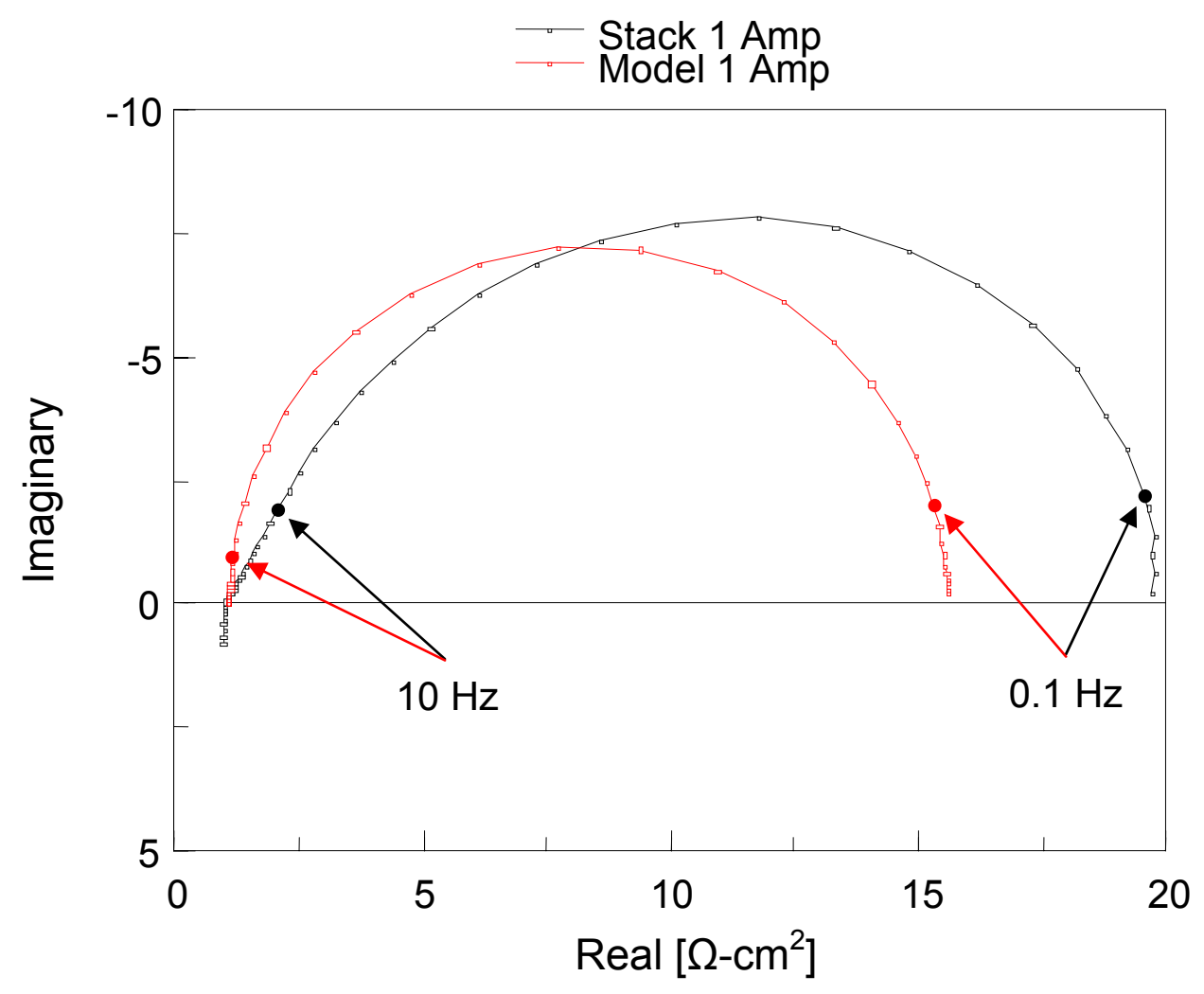

Figure 4.4: Complex Plot of 1A
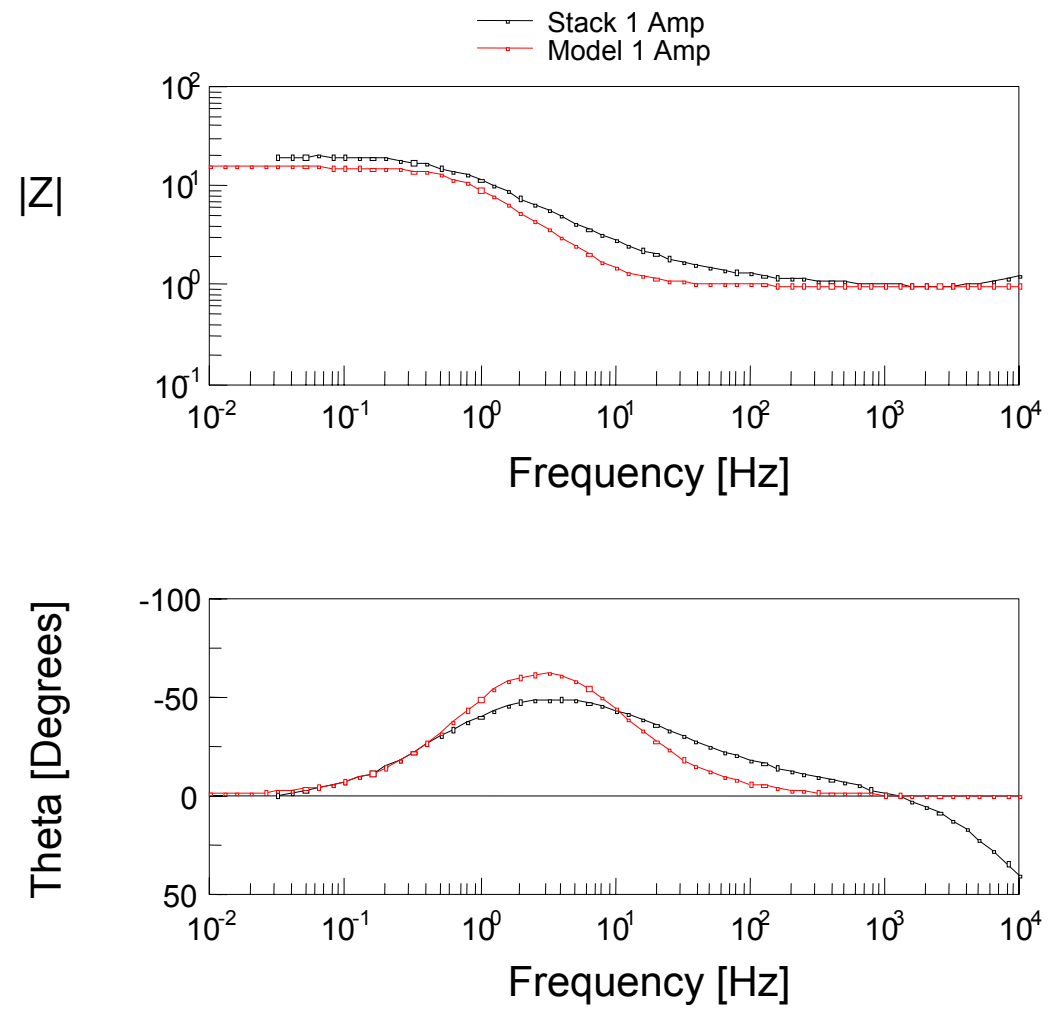

Figure 4.5: Bode Plot of 1A 


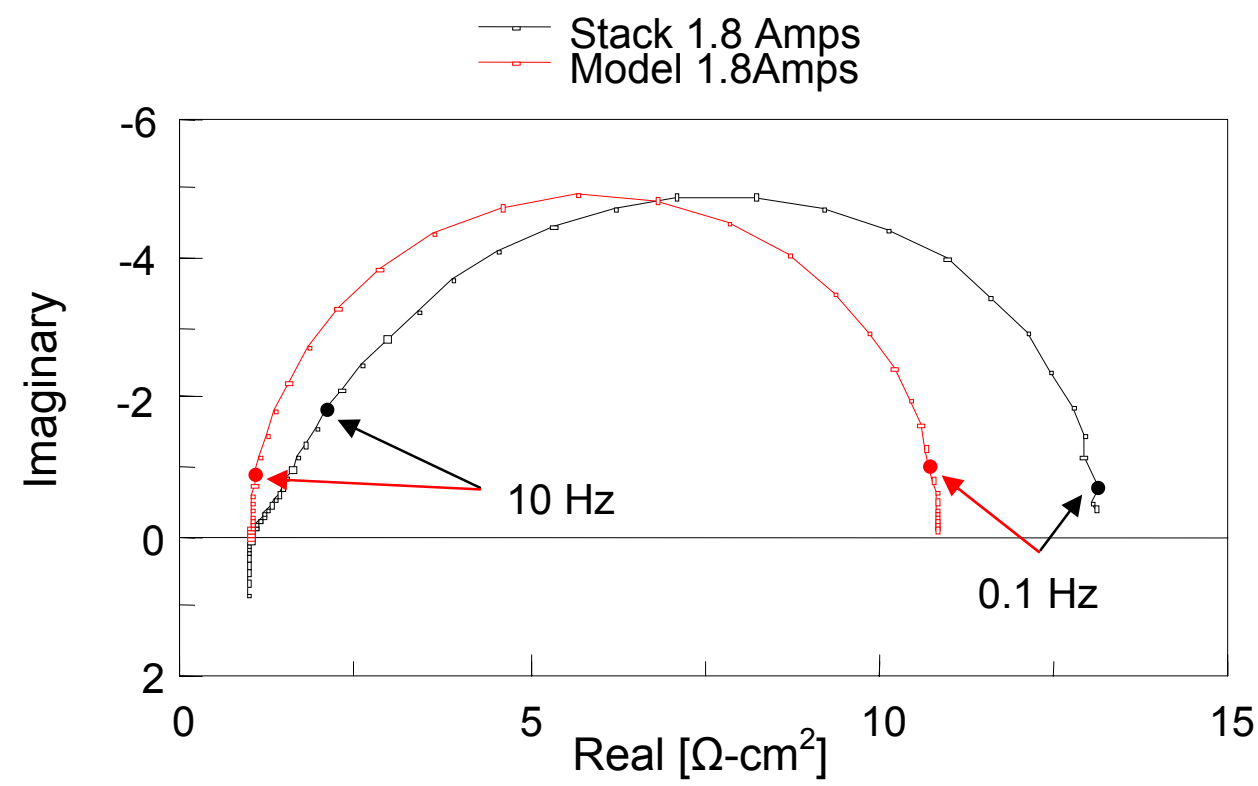

Figure 4.6: Complex Plot of 1.8A
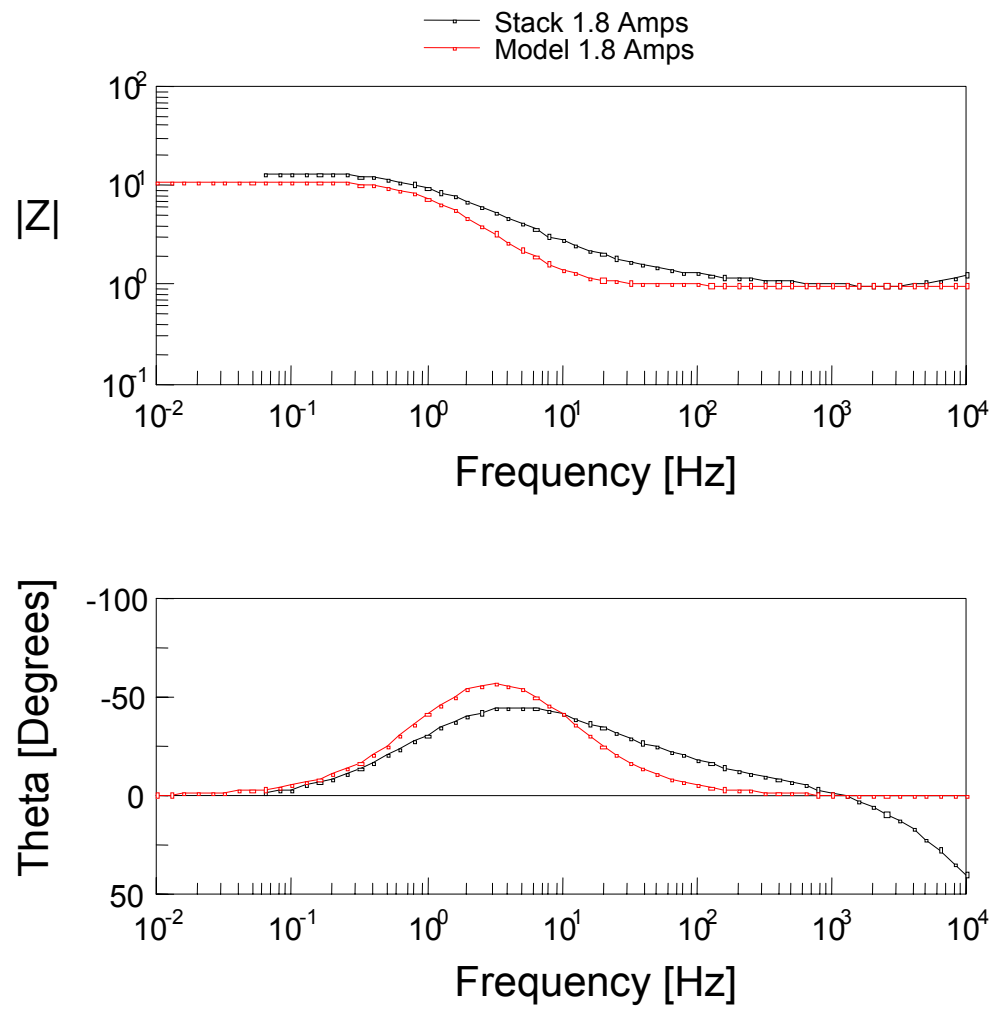

Figure 4.7: Bode Plot of 1.8A 


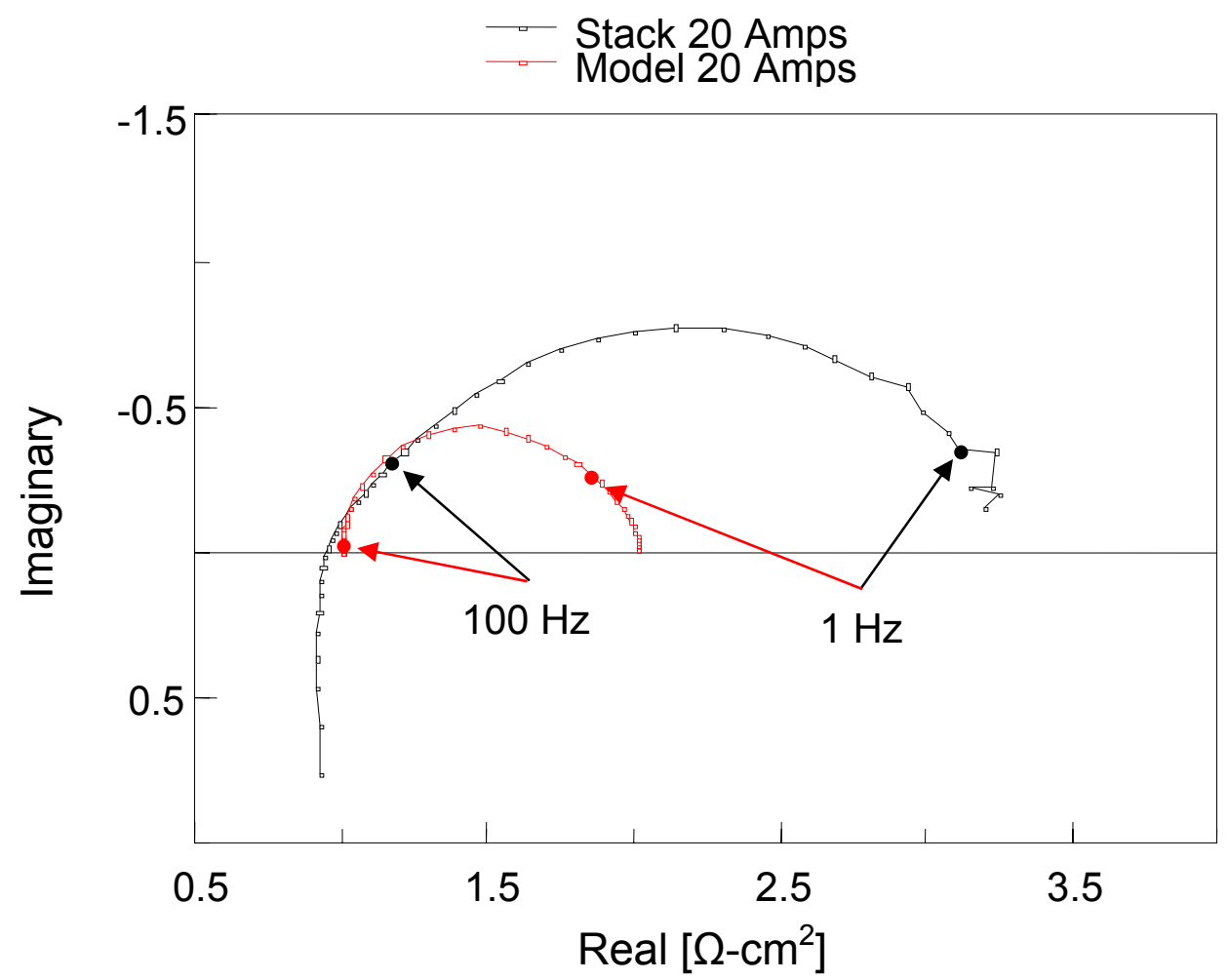

Figure 4.8: Complex Plot of 20A
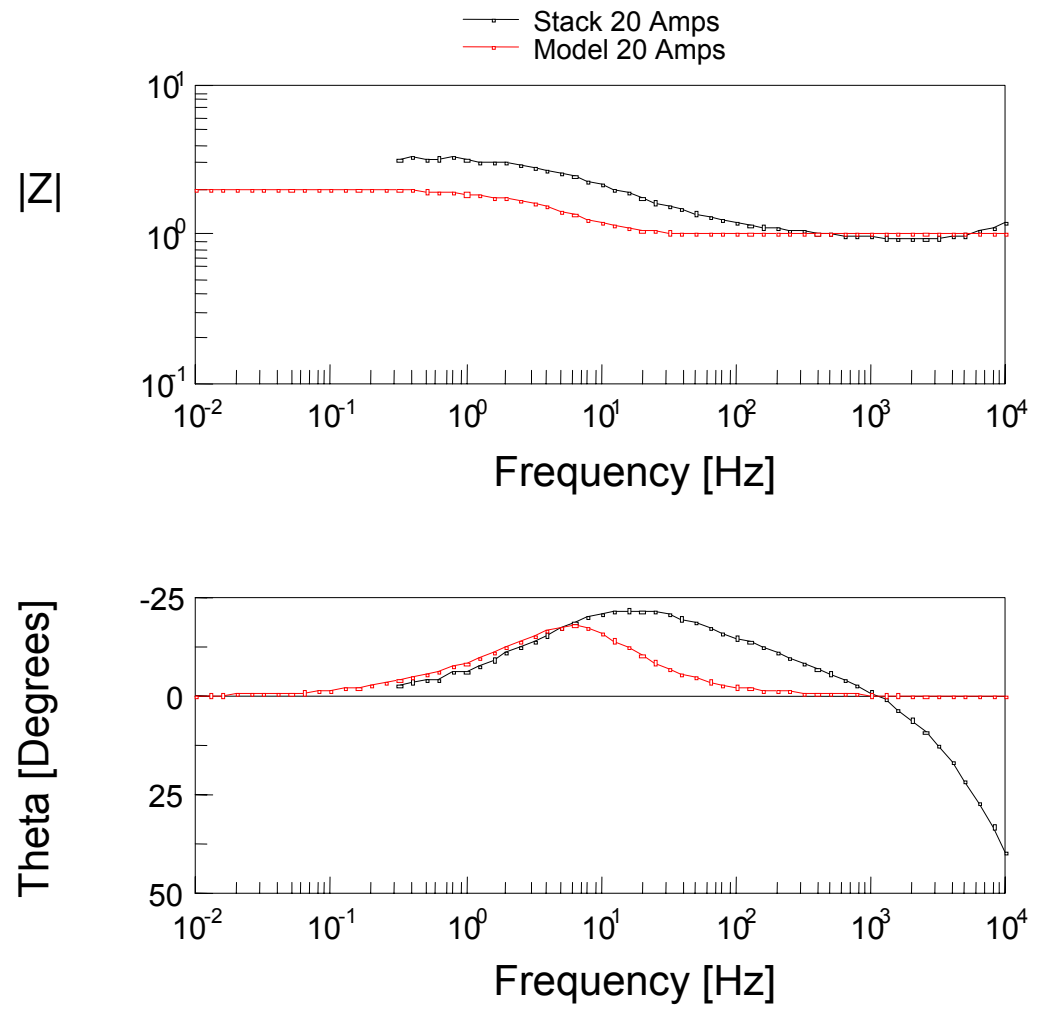

Figure 4.9: Bode Plot of 20A 

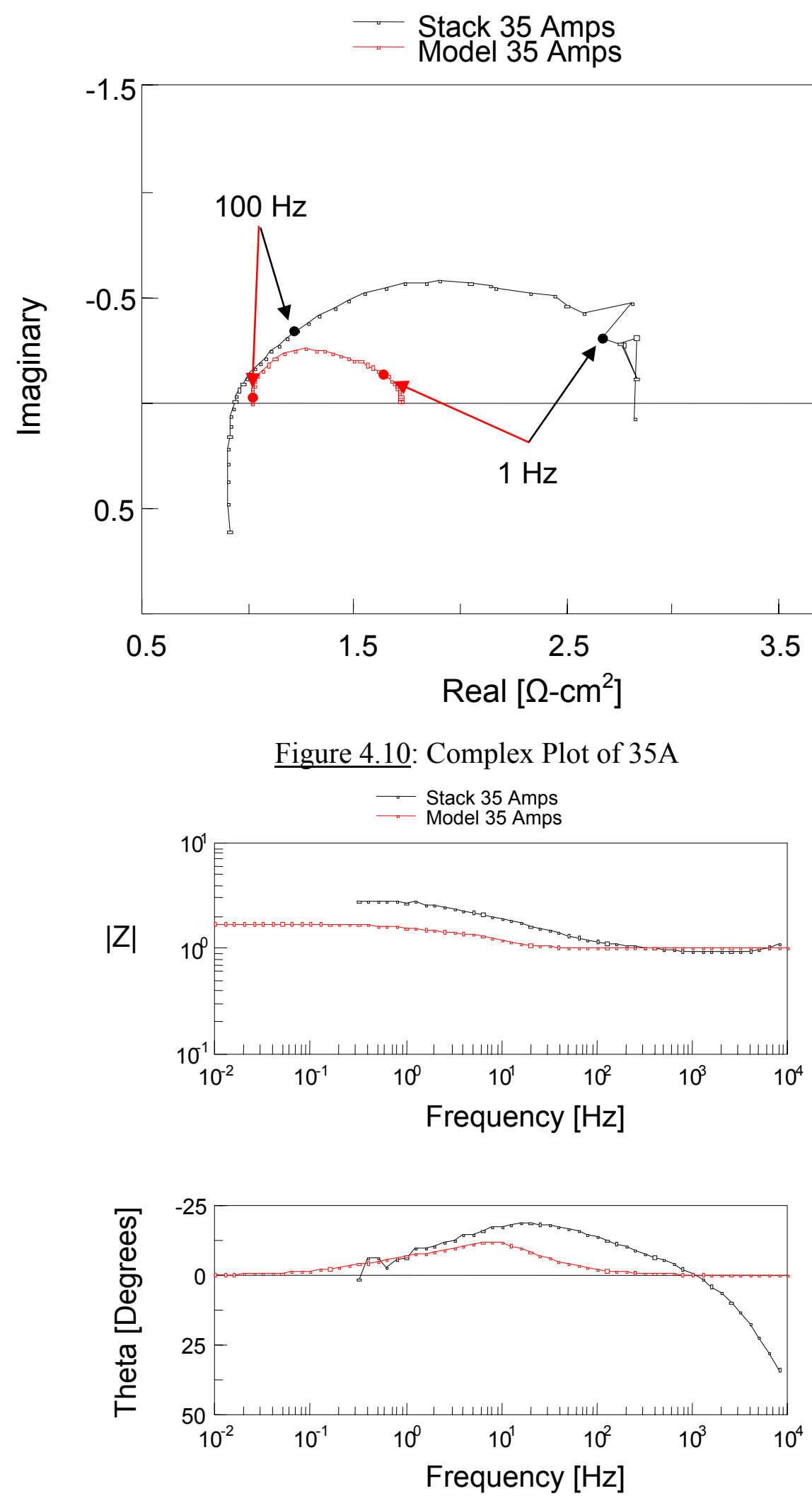

Figure 4.11: Bode Plot of 35A 


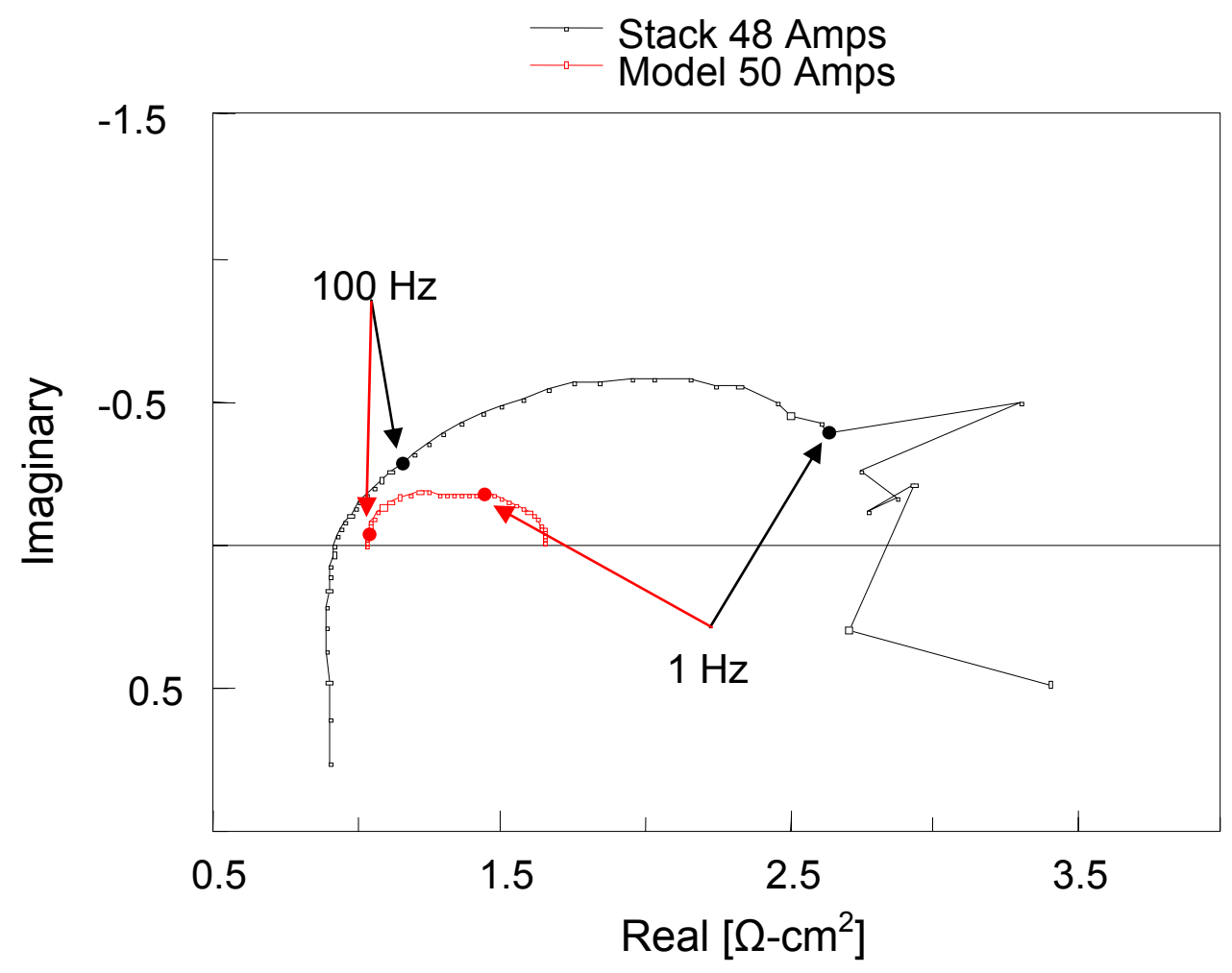

Figure 4.12: Complex Plot of 50A
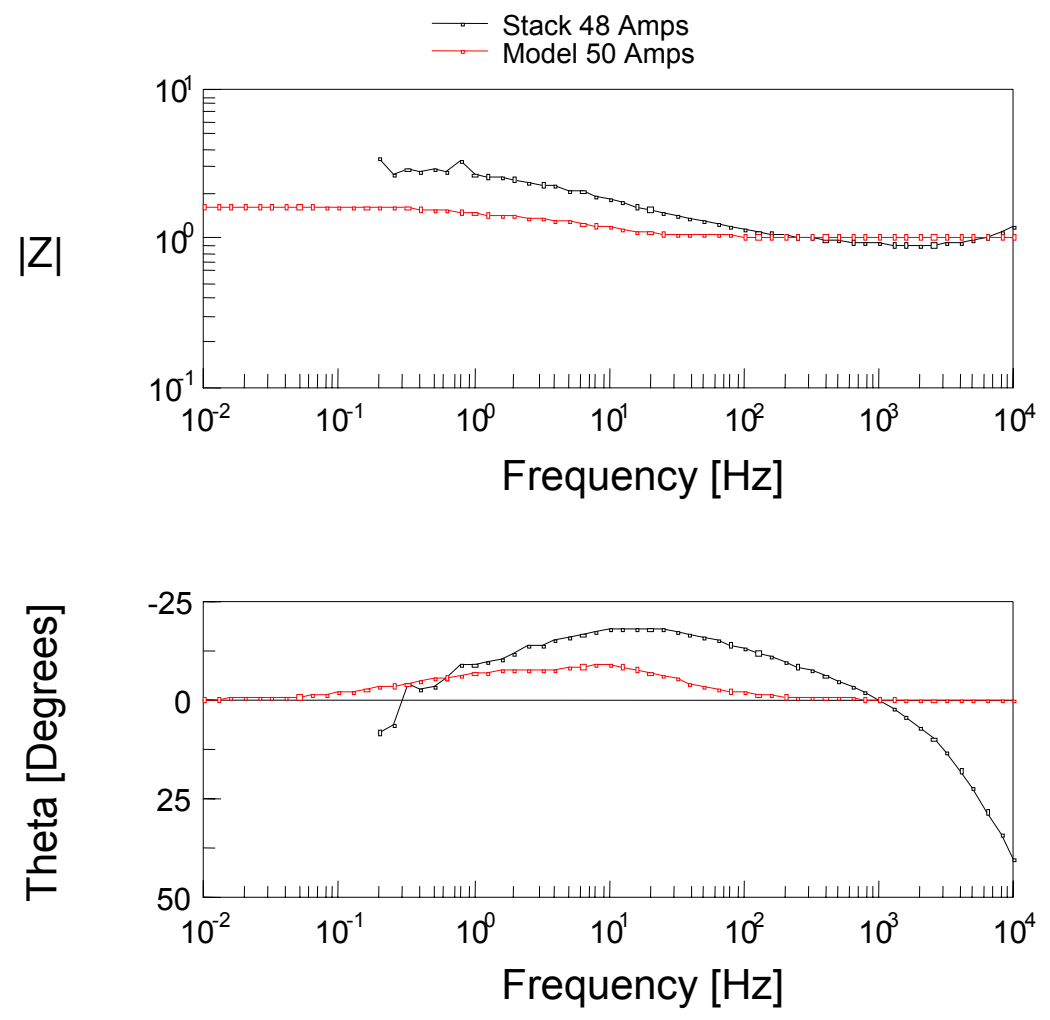

Figure 4.13: Bode Plot of 50A 


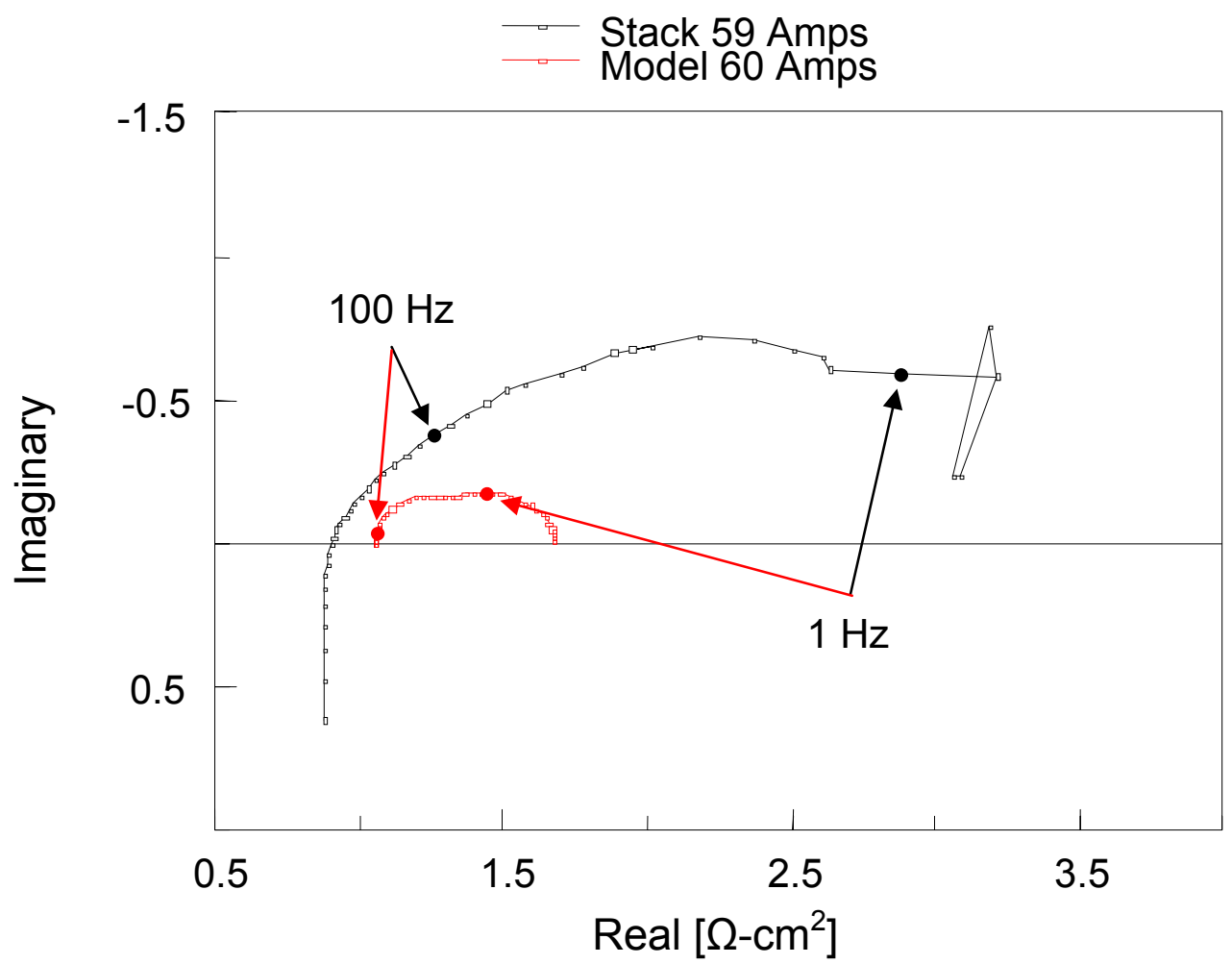

Figure 4.14: Complex Plot of 60A
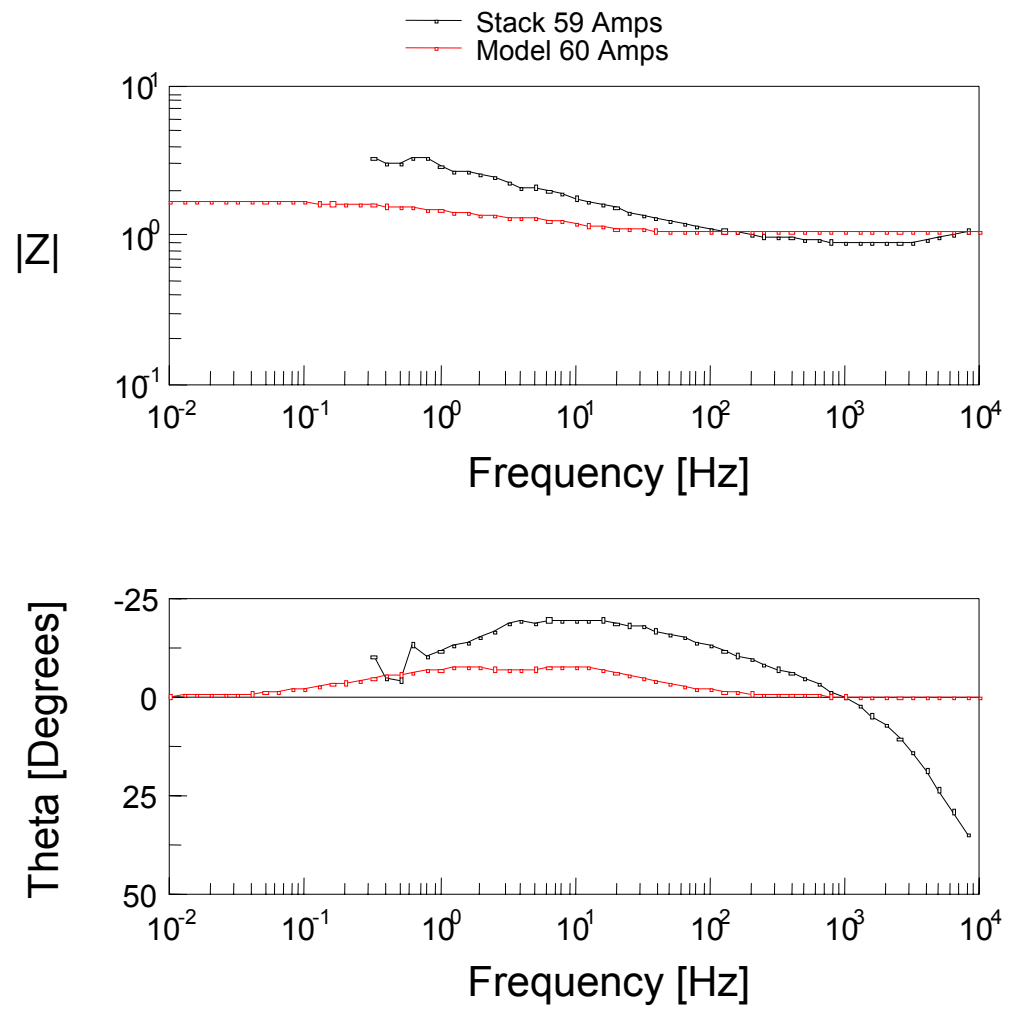

Figure 4.15: Bode Plot of 60A 


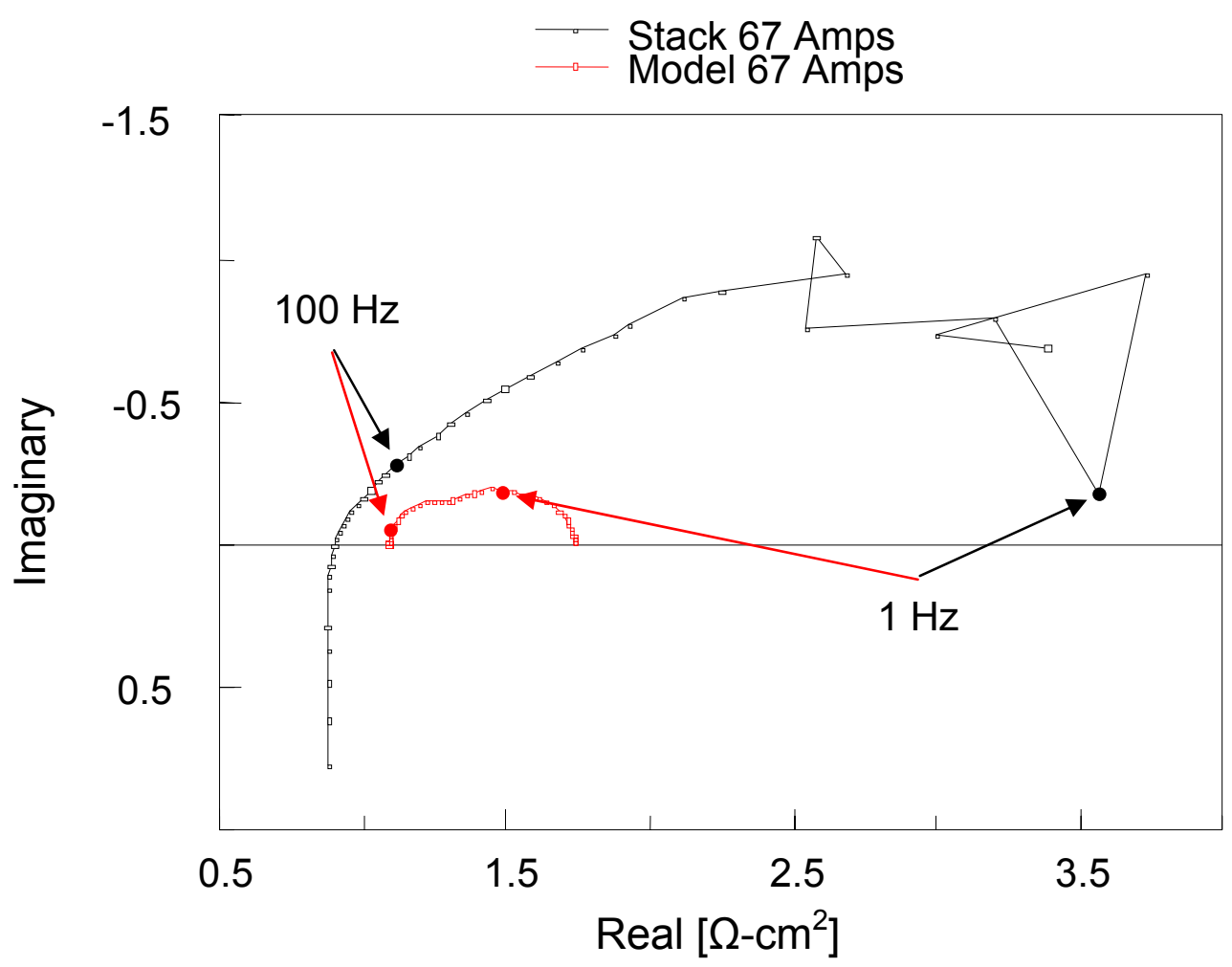

Figure 4.16: Complex Plot of 67A
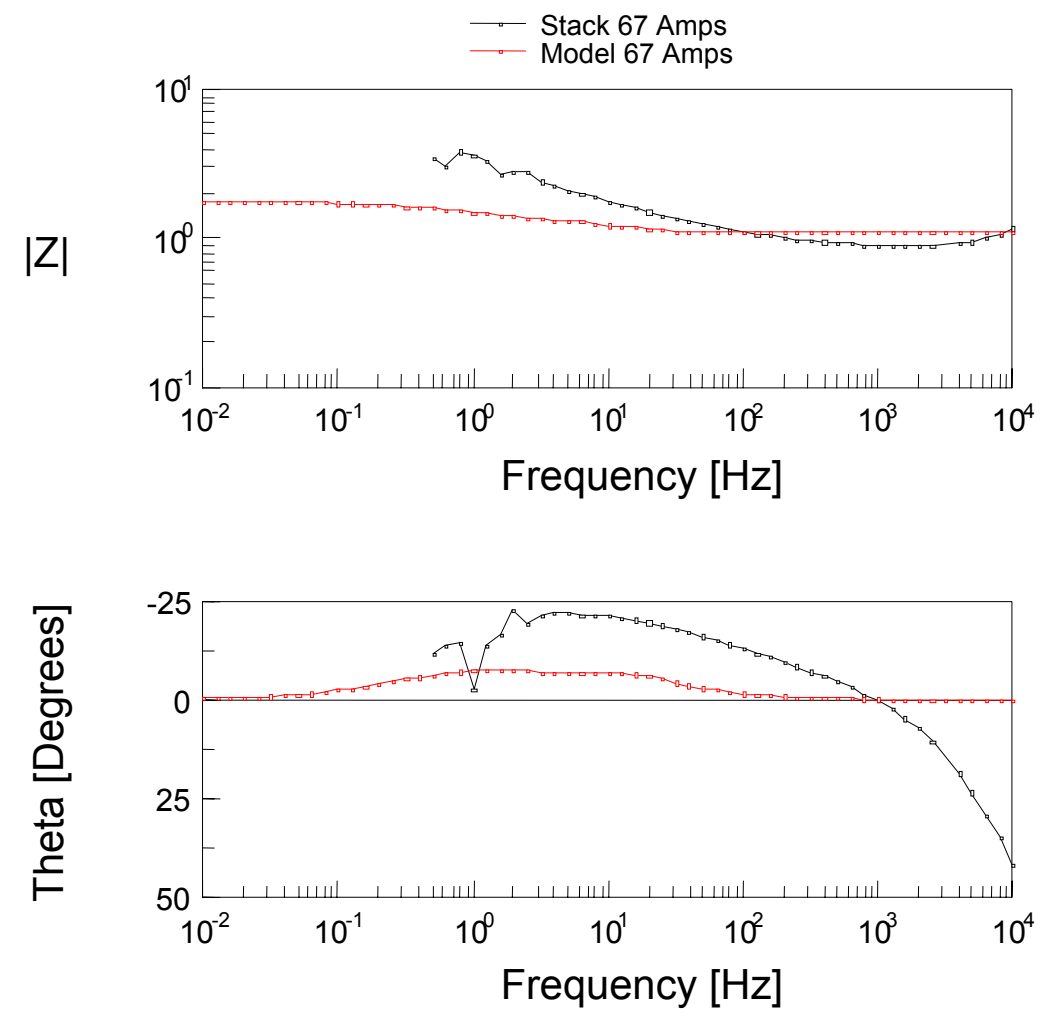

Figure 4.17: Bode Plot of 67A 
From the comparisons, it can be seen that the model always underestimates the magnitude of the low frequency response. Also of note, the experiment and model show opposite effects on the ohmic resistance. The experimental data shows a decreasing resistance with increasing stack current, while the model shows an increase. While the model simulations are always composed of at least one semicircle, the experimental data appears to maintain a single arc, which becomes increasingly depressed with higher currents.

The data for both experiment and model are summarized below in three dimensional Bode plots, showing how the response changes with the DC current. The Bode magnitude plots from the experiment and model are grouped together in Figures 4.18 and 4.19, respectively. Also, the Bode phase plots are grouped together in Figures 4.20 and 4.21 . 


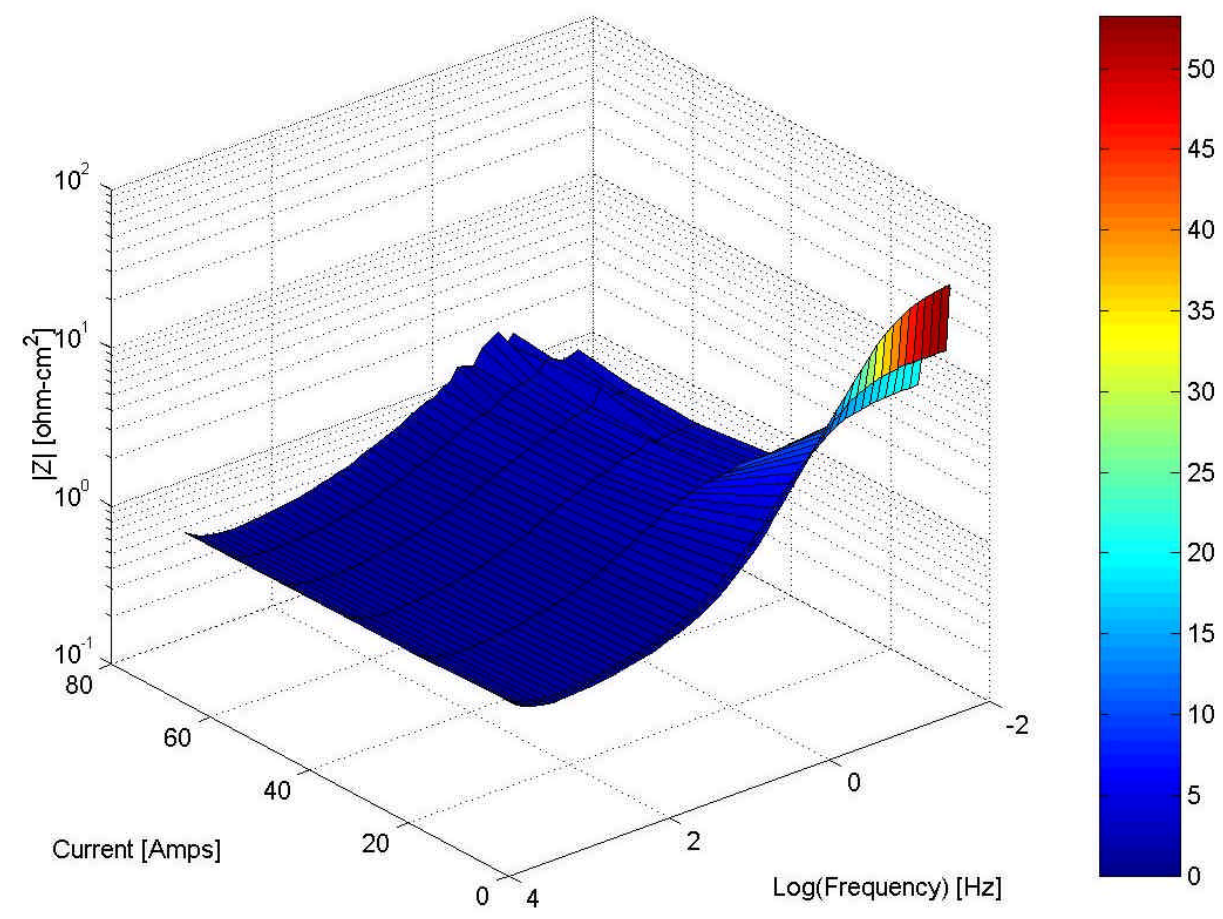

Figure 4.18: 3D Bode Magnitude of all Experimental PEMFC Stack Measurements

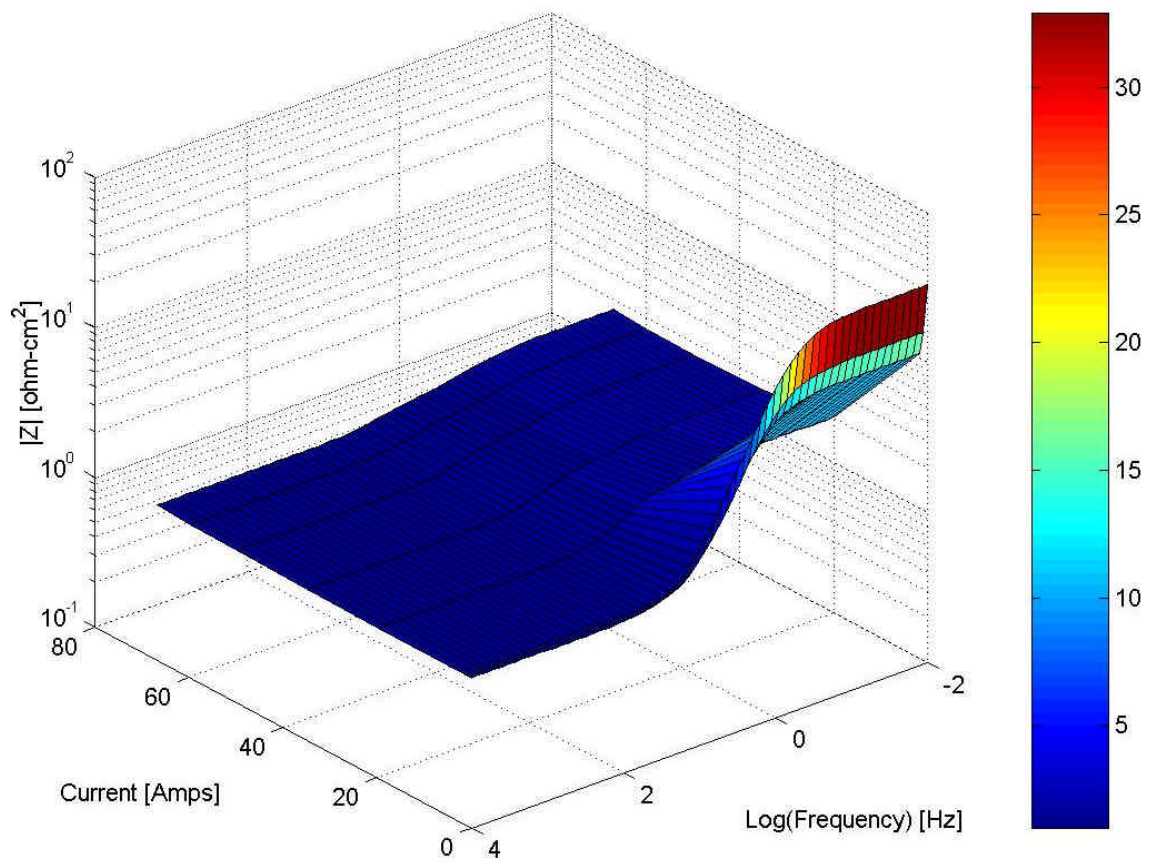

Figure 4.19: 3D Bode Magnitude of all PEMFC Stack Model Simulations 


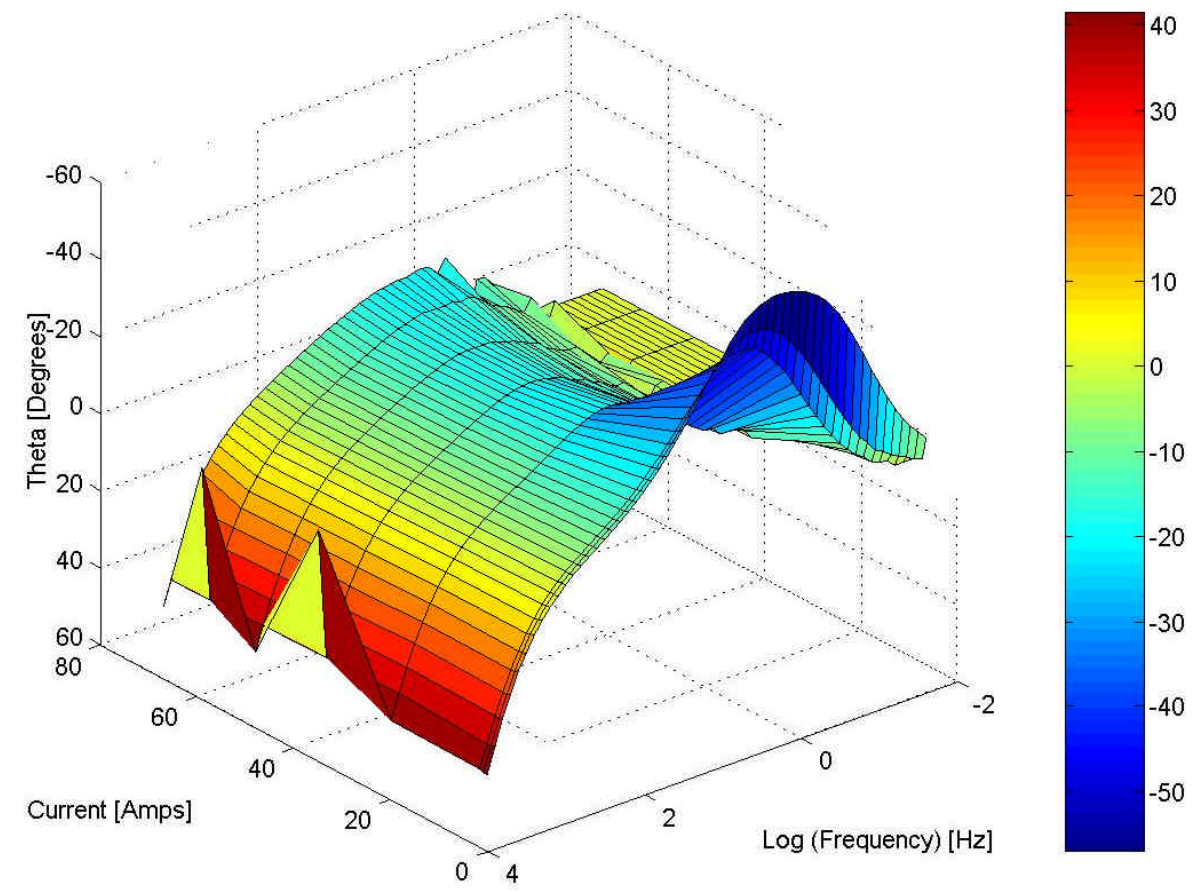

Figure 4.20: 3D Bode Phase of all Experimental PEMFC Stack Measurements

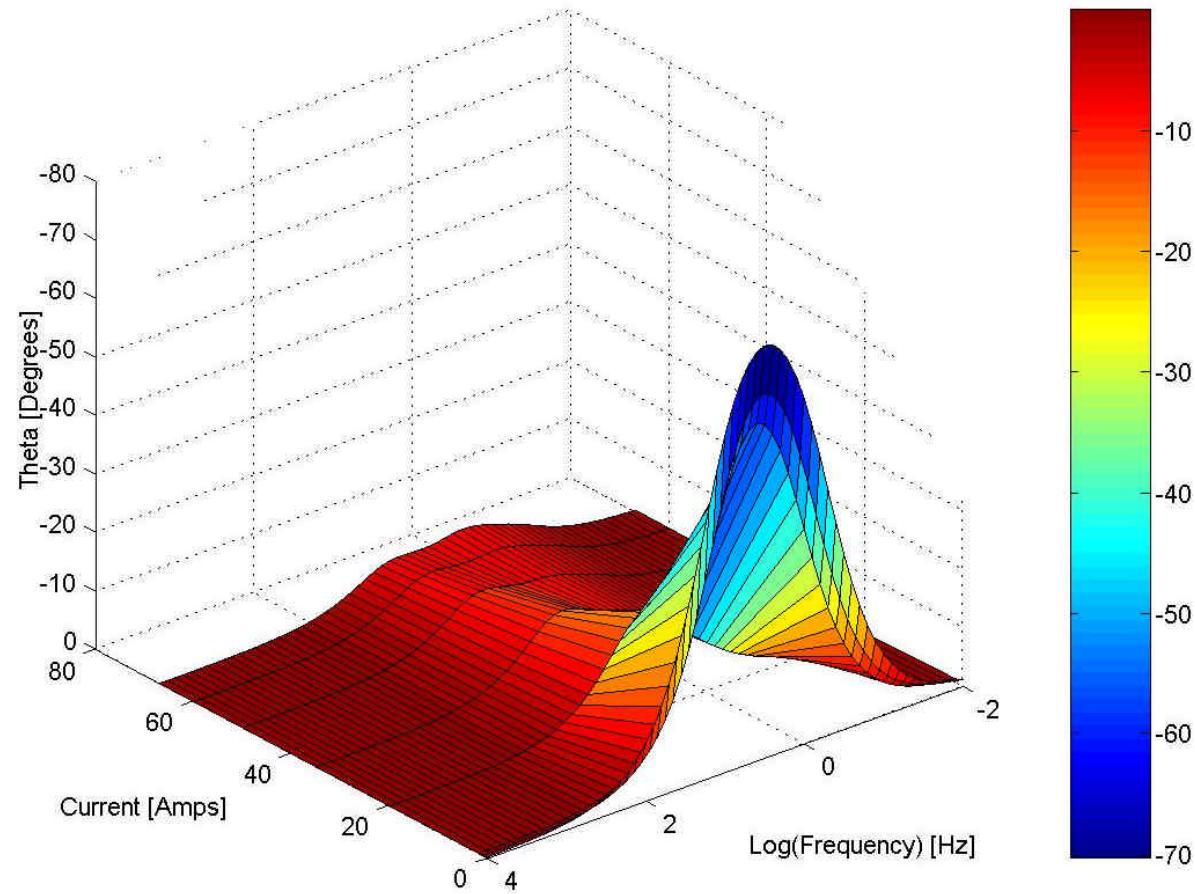

Figure 4.21: 3D Bode Phase of all PEMFC Stack Model Simulations 
In a very general sense it can be seen that the model and experiment show similar responses to changes in current. The overall trend of how the data changes with current can be seen from the three-dimensional Bode Plots. Both experiment and model produce an initial large, single arc result in the complex plane. As the current increases, this response quickly becomes much smaller (by over a factor of 10), and then starts to gradually increase for both cases. Similarly, there is also a trend present in the phase information obtained from both cases. As the current increases, the maximum phase angle shifts to lower frequencies. In the model, there are two arcs that cause this effect, one at high frequencies which is diminishing, while the other arc at low frequencies is growing. By contrast, in the experimental data only one arc is distinguishable and appears to shift the maximum phase angle to lower frequencies.

\subsection{Observations}

While there are definite discrepancies between the experimental data and the model simulations, there are still some important observations that can be made. The presence of similar trends in both the experiment and model shows that the model is capable of simulating the fundamental behavior of the fuel cell stack. This is significant because of the simplicity of the bulk model. The model, which is quite simplified, can still predict the nature of the response of the stack as it changes with load current.

Regarding the experimental response, if the arc in the response is produced from a single effect, the depressed arc can be modeled with an equivalent circuit consisting of a constant phase element (CPE) and a resistor in parallel. A CPE is a more general form of a capacitor, with a capacitor being a CPE with a constant phase difference of $90^{\circ}$. A capacitor produces a semicircle response, which is how the model simulates the response. A depressed arc comes from a constant phase difference of less than $90^{\circ}$, which is similar to the experimental response. A possible reason for the CPE response in the experimental data could be due to the capacitance in the catalyst layer being distributed rather than at a single interface [51]. Another explanation of the depressed arc could be that the experimental arc is combination of the response from several cells which are not identical. This could result in a blending of all the cell responses summed together in the same way Ciureanu [29] suggested that a single cell response could be comprised of 
several indistinguishable arcs. The result could then appear as a single arc that has been altered due to the combination of several similar, but not identical, arcs (one from each cell). In addition, the highly depressed nature of the higher current responses might even suggest that the cells are possibly beginning to show two or more separate arcs, as the model predicts. These arcs would also be intermingled together, complicating the response even further. More testing, under varying conditions, would be required to determine if this situation was occurring.

The model, at present, does not offer much insight into the interpretation of the measured response, since it generally lumps parameters together into a bulk quantity. Thus, it cannot generate the kind of responses proposed by the first suggested explanation of the depressed arc. Also, since the model's response was intended to match the experimental stack's response, both of which assumed four identical cells, the second possibility was not examined. However, the model has demonstrated it could potentially provide more information, pending improvements and revisions, by capturing the overall trend present in the EIS responses.

The difference between experiment and model regarding how the ohmic resistance changes with current could be explained by results published by Bender, et al. [52]. They reported the high frequency resistance initially decreased with increasing current then started to increase with a further increase in current. This is sketched in Figure 4.22. 


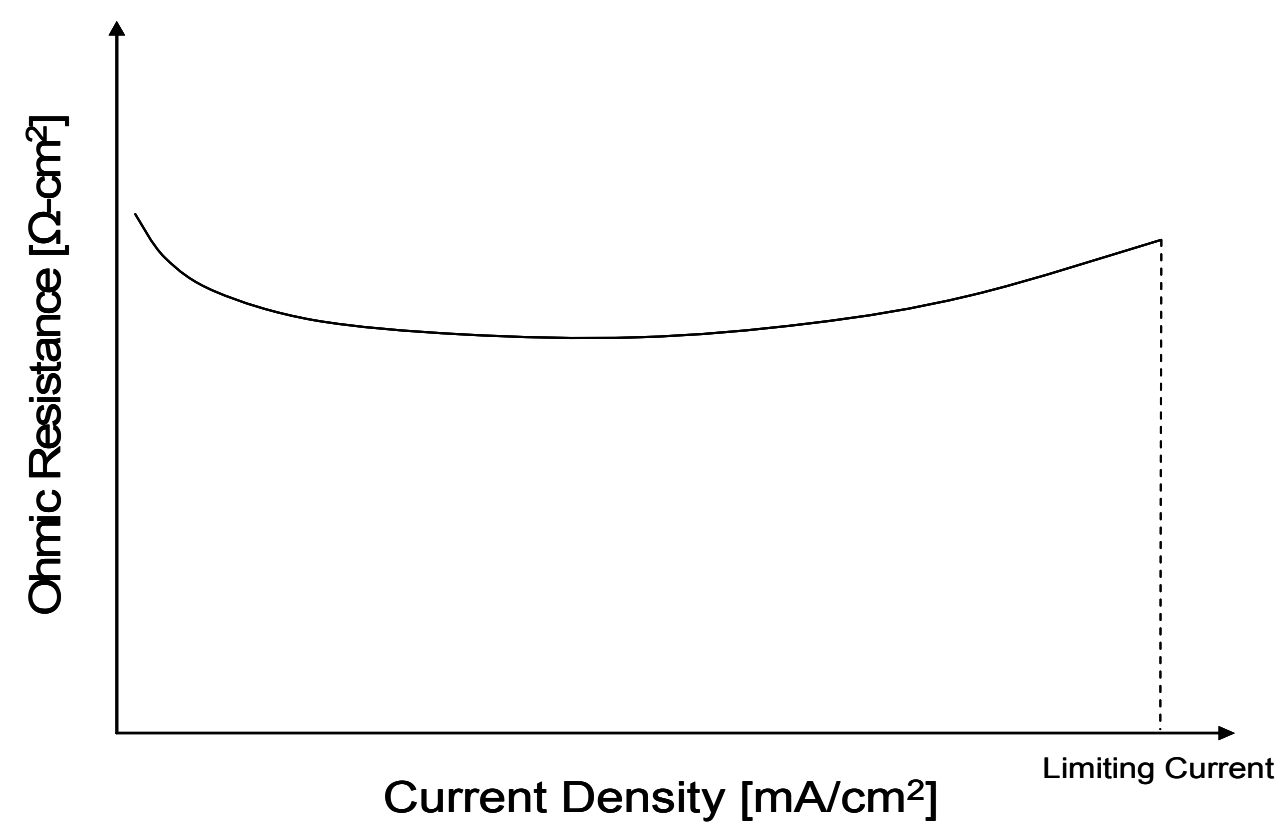

Figure 4.22: Effect of Current on Ohmic Resistance of Fuel Cell [52]

Bender et al. attribute the initial decrease in ohmic resistance to an improvement in membrane conductivity due to better membrane hydration. They claim the increase in resistance may be due to the anode side of the membrane drying out, thus reducing conductivity. This could explain why the experimental EIS data shows a decrease in the ohmic resistance with increasing current. The reason an increase was never observed in this work could be explained by noting the current density when the measurements were taken. The maximum current density for which a response was measured was approximately $0.5 \mathrm{~A} / \mathrm{cm}^{2}$. From previous measurements [44], the stack reaches a limiting current density of approximately $0.82 \mathrm{~A} / \mathrm{cm}^{2}$. It is not known how the ohmic resistance of the stack changes as the current approaches the limiting current density. The reason the model ohmic resistance increases is due to increased diffusion losses through the gas diffusion electrodes (cf. Equation 3.7). The model has no mechanism to account for fluctuations in the membrane conductivity and simply assumes a fixed value.

To check the accuracy of the model simulated response at low currents (where the voltage is mostly determined by activation processes), according to Ciureanu [29], the charge transfer resistance, which is the diameter of the arc at low currents, at open circuit conditions is given by the following equation,

$$
R_{C T}=\frac{R T}{n F i_{o}}
$$


The value for $\mathrm{n}$ is the number of electrons transferred in the process, in this case, two; one from each hydrogen atom in the diatomic molecule. Using the value for the reference exchange current density in the model, $1.7 \mathrm{~mA} / \mathrm{cm}^{2}$, solving Equation 4.1 yields a charge transfer resistance of $8.7 \Omega / \mathrm{cm}^{2}$. Multiplying this by four, for the number of cells in the stack, produces the value $34.8 \Omega / \mathrm{cm}^{2}$. This agrees closely with the value of $31.9 \Omega / \mathrm{cm}^{2}$ for the charge transfer resistance simulated at $0.2 \mathrm{~A}$, which is close to open circuit conditions. Using the same process to estimate an exchange current density from the experimental measurements suggests a value for $i_{o}$ to be less than $1.1 \mathrm{~mA} / \mathrm{cm}^{2}$. This assumes a value for $\mathrm{R}_{\mathrm{CT}}$ of 13.325 , which comes from dividing the diameter of the $0.2 \mathrm{~A}$ arc, $\sim 53.3 \Omega / \mathrm{cm}^{2}$, by four. This exchange current value is similar to the value obtained from the VI curve fit. This result also supports the model in assuming a linear system for the stack response by showing that the stack response is composed of the sum of the cell responses.

Some potential sources of error in the experimental measurements include the large AC magnitude, which could affect the linearity of the EIS measurement, and the measurement accuracy of the VI curve, which would affect the model calibration. Also of significance is the change in cell temperatures with current that is not included in the model.

In summary, the model and experiment responses both show similar behavior when the stack current is changed, and generally agree with results published in the literature. However, the overall accuracy is not very high, generally only within the same order of magnitude. The results also show that even though the model showed reasonable agreement in matching the VI curve, it was unable to predict the EIS response with the same degree of accuracy. This emphasizes the need to use another measure, other than just the VI curve, to provide input to a model, and thereby come to a better understanding of the processes at work within a fuel cell stack. It is hoped that EIS can fulfill this role for stack applications. 


\section{Chapter 5: Conclusions and Future Work}

\subsection{Conclusions}

The primary goal of this thesis was to compare Electrochemical Impedance Spectroscopy measurements performed on an operating PEM fuel cell stack to a response simulated by a model of the stack. This objective was met as measurements of the stack's response were compared to the model's response over a range of current loads. The stack and model responses showed qualitative agreement, although they did not match exactly. The results show that the model predicted well the behavior of the VI curve for currents less than 50 Amps. The model also qualitatively predicted the impedance response as a function of current and frequency. Because of apparent model incompleteness, it was not able to predict accurately the shape of the response in the complex plane at currents larger than 20 Amps and did not match the change in ohmic resistance with current.

\subsection{Stack EIS Challenges}

In extending EIS to a PEMFC stack, a couple challenges were encountered with the experimental setup. One of the more significant issues was noise in the measurement response. Much of this noise is attributable to the series connection of the four large surface area cells. This could potentially be a significant problem with stacks consisting of many cells. But for the four cell stack used in this work, the magnitude of the AC signal was increased to improve the signal-to-noise ratio and counteract the increased noise levels. Another challenge was measuring the EIS response at high currents. The resistive load bank provided the best overall solution by not complicating the measurement setup while enabling moderate current densities in the stack. 


\subsection{Future Work}

This section describes some of the extensions of this thesis. First, experimental conditions are suggested. Then, model improvements are discussed. Last, a potential application of EIS as a diagnostic tool for PEMFC stack operation is suggested.

\subsubsection{Experiment}

An extension of this work would measure the response for different operating conditions such as number of cells, gas flow rates and pressure, humidification, temperature, and MEA properties. This would include the case where the conditions were varied across the entire stack and also the case when individual cell conditions are adjusted independent of the overall stack conditions. This would develop a more extensive knowledge of the stack's response at different conditions and could show more relationships between known stack conditions and the measured response. From these relationships, a more detailed and comprehensive model could be developed, which would help improve the interpretation and accuracy of the simulated responses. The ultimate goal of this work would provide the capability to relate the measured response of a stack to information about the conditions of the stack which may not be available from just the individual cell voltages. Then, based on this understanding of its response, the stack could be optimized in order to perform more effectively and efficiently.

\subsubsection{Model}

The fuel cell model could be improved in several ways. To show better agreement when comparing the simulated EIS responses to the measured responses, the bulk model needs to be converted into a distributed model. This would capture some of the distributed effects that are present in the fuel cell stack which contribute to the measured response. Also, the model could use more detailed equations which would better resemble the actual fuel cell stack behavior. By way of example, the stack temperature is set initially in the model and then remains constant throughout simulation. In reality, the temperature of the stack is a function of many variables and fluctuates as the conditions on the stack change. Another example is the cell's double layer capacitance which is always assumed to be constant for all cells. 


\subsubsection{Potential Application}

The likely use of EIS with fuel cell stacks would require an understanding of the general response of the stack, given known operating conditions. The nominal case would produce a stack response that would resemble a single cell response multiplied by the number of cells, given that all cells are approximately equal and are operating properly. Therefore, if the response was different from the nominal case, a potentially bad cell could be identified based on the effect it would have on the stack response. This is not to say that the cell number could be determined, but, rather, this is a collective check on the performance of all the cells to see if they are operating normally. As a simple example, suppose the following equivalent circuit represented a four cell stack, where $\mathrm{R} 1$ represents the sum of all the ohmic resistances, and each cell is reduced to a RC pair.

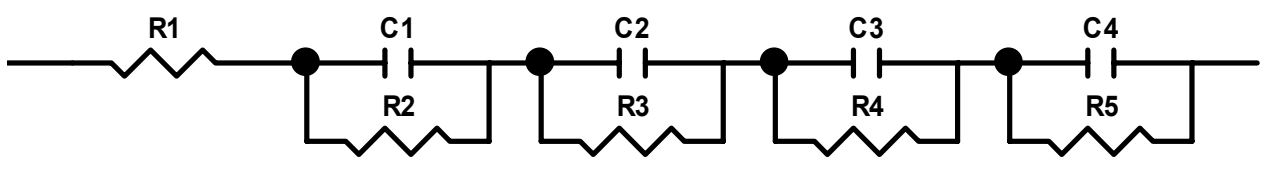

Figure 5.1: Simple Fuel Cell Stack Equivalent Circuit

For simplicity, all values for resistors are $1 \Omega$ and all values of capacitors are $1 \mathrm{~F}$. The response of such a stack would be depicted by the 'Simple Stack' response in Figures 5.2 and 5.3. To illustrate the change in the stack response by a single cell changing, one of the parallel resistors was increased to $10 \Omega$ in the response 'Simple Stack High Resistance Cell'. The response 'Simple Stack High Capacitance Cell' involved replacing one of the $1 \mathrm{~F}$ capacitors with a $4 \mathrm{~F}$ capacitor. All other circuit elements remained unchanged.

Specifying which cell(s) has the problem and identifying the nature of the problem are beyond the scope of this thesis. A detailed study of fuel cell stack EIS responses would need to be performed to validate this potential application of EIS measurements. 


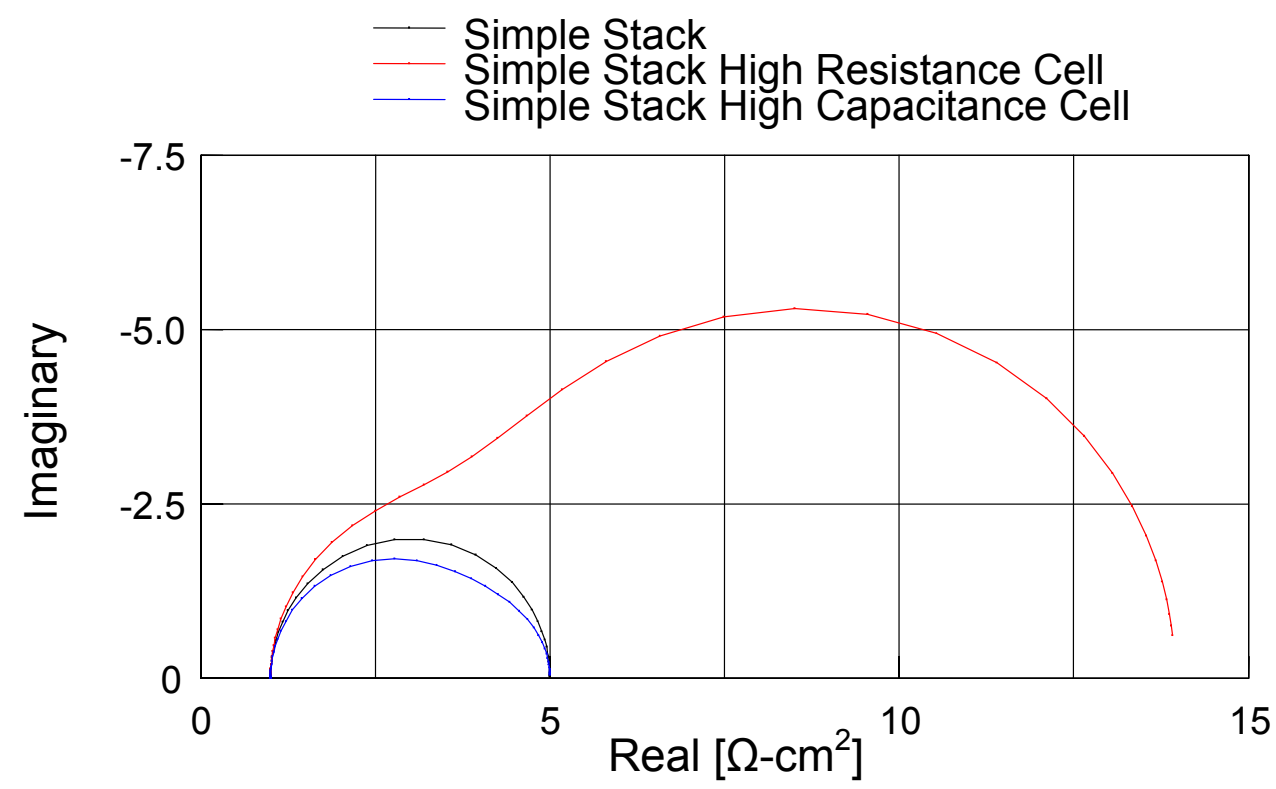

Figure 5.2: Responses of Simple Stack Example
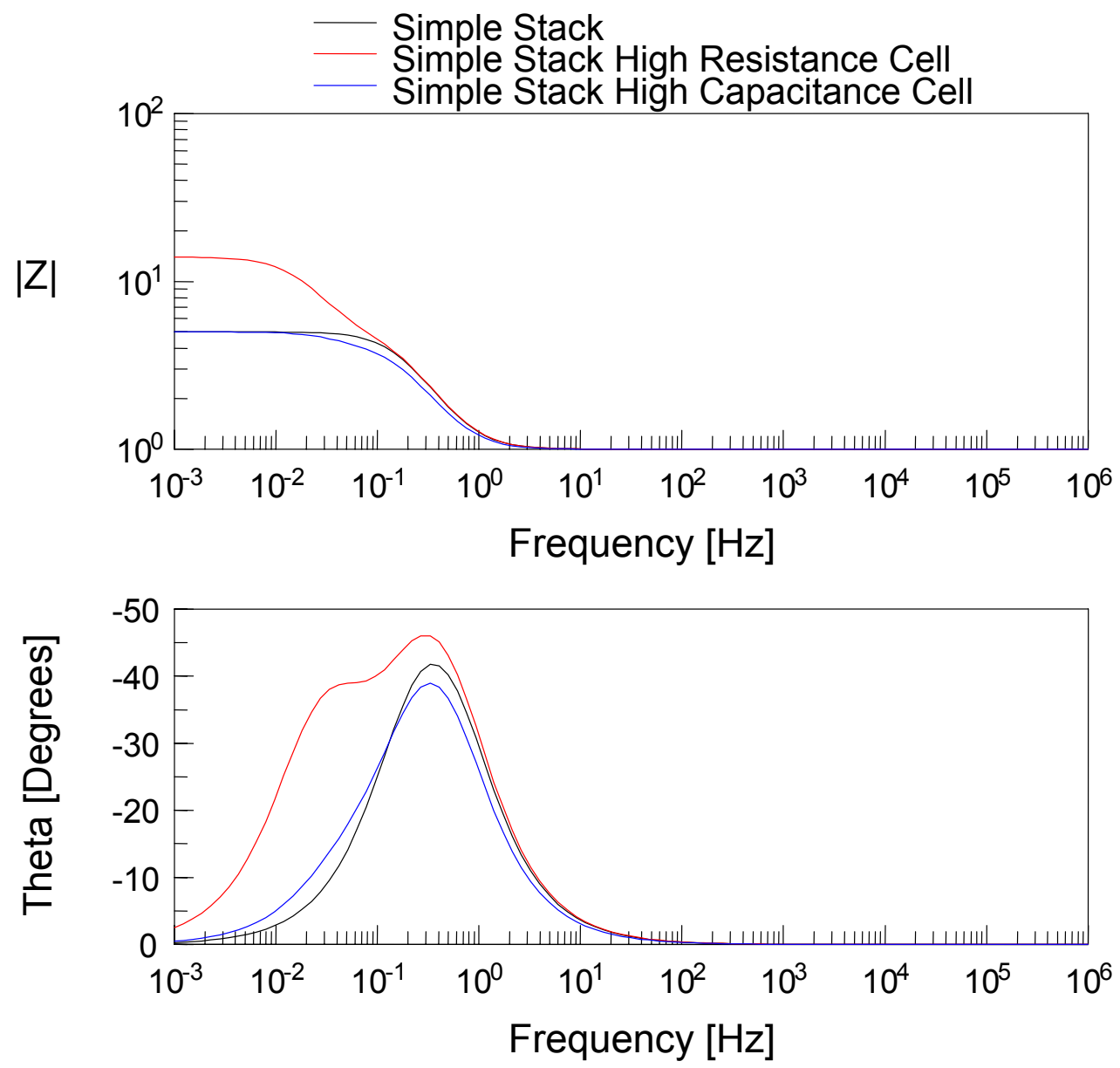

Figure 5.3: Bode Plot of Simple Stack Example 


\section{References}

[1] Abraham, Spencer, "Secretary Abraham Outlines National Energy Policy", http://www.energy.gov/engine/content.do?PUBLIC_ID $=13409 \& B T$ CODE=PR_S PEECHES\&TT CODE=PRESSSPEECH, Accessed July 3, 2004

[2] C. Stone, A.E. Morrison, Solid State Ionics 152-153 (2002) 1.

[3] J.M. Ogden, M.M. Steinbugler, T.G. Kreutz, J. Power Sources 79 (1999) 143.

[4] M.G.L. Santarelli, M. Calì, A. Bertonasco, Energy Conversion and Management 44 (2003) 2353.

[5] J. Hamelin, K.Agbossou, A. Laperrière, F. Laurencelle, T.K. Bose, Intl. J. Hydrogen Energy 26 (2001) 625.

[6] D.W. Green, Editor, Perry's Chemical Engineers' Handbook, $7^{\text {th }}$ Ed., McGrawHill, New York, NY, 1997

[7] A. Parthasarathy, B. Dave, S. Srinivasan, and A.J. Appleby, J. Electrochemical Soc. 139 (1992) 1634.

[8] T. Mennola, M. Mikkola, M. Noponen, T. Hottinen, and P. Lund, J. Power Sources 112 (2002) 261.

[9] S.-Y. Ahn, S.-J. Shin, H.Y. Ha, S.-A. Hong, Y.-C. Lee, T.W. Lim, I.-H. Oh, J. Power Sources 106 (2002) 295.

[10] N.P. Siegel, M.W. Ellis, D.J. Nelson, M.R. von Spakovsky, J. Power Sources, 115 (2003) 81.

[11] M.E. Orazem, P. Agarwal, and L.H. Garcia-Rubio, J. Electroanalytical Chemistry 378 (1994) 51.

[12] T.A. Zawodzinski, T.E. Springer, F. Uribe, and S. Gottesfeld, Solid State Ionics 60 (1993) 199.

[13] M. Wakizoe, O.A. Velev, and S. Srinivasan, Electrochimica Acta 40 (1995) 335.

[14] C.A. Ward and J.A. Garcia, J. Power Sources 66 (1997) 83.

[15] J.A. Garcia, C.A. Ward, R.D. Venter, and S. Ho, J. Power Sources 66 (1997) 89.

[16] V. Mehta and J.S. Cooper, J. Power Sources 114 (2003) 32.

[17] R. Jiang and D. Chu, J. Power Sources 93 (2001) 25.

[18] D. Chu and R. Jiang, J. Power Sources 80 (1999) 226.

[19] D. Chu and R. Jiang, J. Power Sources 83 (1999) 128.

[20] Z. Qi and A. Kaufman, J. Power Sources (2002) 469.

[21] N.D. Cogger and N.J. Evans, Solartron Technical Report No. 6 (1999).

[22] N.D. Cogger and R.V. Webb, Solartron Technical Report No. 10 (1997).

[23] A.M. Kauffman, Solartron Technical Report No. 17 (1997).

[24] S.R. Taylor, et al., A Short Course on Electrochemical Impedance Spectroscopy, The Center for Electrochemical Science and Engineering, U. of Virginia, (2002).

[25] T.E. Springer, T.A. Zawodzinski, M.S. Wilson, and S. Gottesfeld, J. Electrochemical Soc. 143 (1996) 587.

[26] V.A. Paganin, C.L.F. Oliveira, E.A. Ticianelli, T.E. Springer, and E.R. Gonzalez, Electrochimica Acta 43 (1998) 3761.

[27] T.J.P. Freire and E.R. Gonzalez, J. Electroanalytical Chemistry 503 (2001) 57. 
[28] N. Wagner, W. Schnurnberger, B. Muller, and M. Lang, Electrochimica Acta 43 (1998) 3785.

[29] M. Ciureanu and R. Roberge, J. Physical Chemistry B 105 (2001) 3531.

[30] I.D. Raistrick, Electrochimica Acta, 35 (1990) 1579.

[31] B. Andreaus, A.J. McEvoy, and G.G. Scherer, Electrochimica Acta 47 (2002) 2223.

[32] J.-P. Diard, N. Glandut, P. Landaud, B. Le Gorrec, and C. Montella, Electrochimica Acta 48 (2003) 555.

[33] T.E. Springer and I.D. Raistrick, J. Electrochemical Soc. 136 (1989) 1594.

[34] T.E. Springer, M.S. Wilson, and S. Gottesfeld, J. Electrochemical Soc. 140 (1993) 3513.

[35] D.M. Bernardi and M.W. Verbrugge, J. Electrochemical Soc. 139 (1992) 2477.

[36] D. Singh, D.M. Lu, and N. Djilali, Int. J. Engineering Science 37 (1999) 431.

[37] T. Berning, D.M. Lu, and N. Djilali, J. Power Sources 106 (2002) 284.

[38] G. Maggio, V. Recupero, and L. Pino, J. Power Sources 101 (2001) 275.

[39] L. You and H. Liu, Int. J. Hydrogen Energy 26 (2001) 991.

[40] J.H. Lee, T.R. Lalk, and A.J. Appleby, J. Power Sources 70 (1998) 258.

[41] J.H. Lee and T.R. Lalk, J. Power Sources 73 (1998) 229.

[42] D. Chu, R. Jiang, and C. Walker, J. Applied Electrochemistry 30 (2000) 365.

[43] R. Jiang and D. Chu, J. Power Sources 92 (2001) 193.

[44] J.P. Hensel, R.S. Gemmen, B.J. Hetzer, J.D. Thornton, J.S. Vipperman, W.W. Clark, and A.F. Ayhan, Proceedings of $2^{\text {nd }}$ Intl. Conference on Fuel Cell Science, Engineering and Technology, Rochester, NY, (2004) 283.

[45] ZPlot for Windows, Operating Manual Version 2.2, Scribner Associates, Inc., 2000.

[46] ZView for Windows, Operating Manual Version 2.2, Scribner Associates, Inc. 2000.

[47] T. Romero-Castañón, L.G. Arriaga, U. Cano-Castillo, J. Power Sources 118 (2003) 179.

[48] R.S. Gemmen, J. Fluids Engineering 125 (2003) 576.

[49] D.M. Bernardi, M.W. Verbrugge, AICHE Journal 37 (1991) 1151.

[50] Z.H. Wang, C.Y. Wang, and K.S. Chen, J. Power Sources 94 (2001) 40.

[51] J.Ross MacDonald, Editor, Impedance Spectroscopy, John Wiley and Sons, New York, NY, 1987.

[52] G. Bender, M.S. Wilson, T.A. Zawodzinski, J. Power Sources, 123 (2003) 163. 


\section{Appendix: Code Listing}

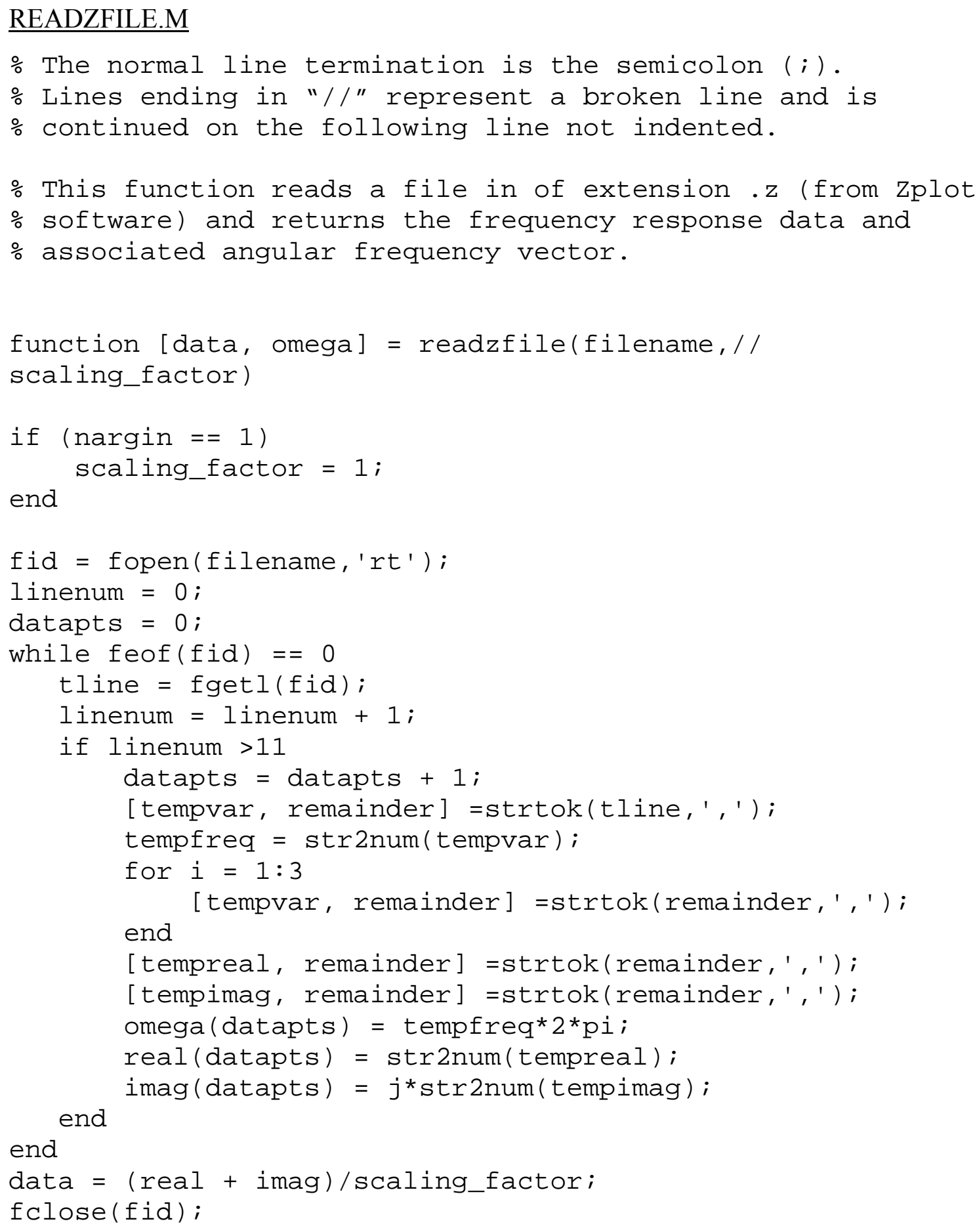




\section{SUBTRACTLOAD.M}

\% The normal line termination is the semicolon (;).

\% Lines ending in "//" represent a broken line and is

o continued on the following line not indented.

:This function will read in two text files, the first

o being the measured stack and load response. The second

ㄷle is the measured load response. This function

\% assumes that the stack and load has been normalized with

\% a scaling factor to account for the cell area and the

o load measurement has not been scaled.

\% 'sandl' stands for Stack AND Load, which is the measured \% fuel cell data operating with a load

:This function requires the function readzfile to execute \% properly.

function stack_sys = subtractload//

(stackandload_filename,load_filename)

[sandl_data, sandl_freqw] $=/ /$

readzfile (stackandload_filename,133.7);

[load_data, load_freqw] = readzfile(load_filename,1);

\% Drop $100 \mathrm{kHz}-12.589 \mathrm{kHz}$ range from load data

load_data = load_data $(11$ :length (load_data) $)$;

load_freqw = load_freqw $(11:$ length (load_freqw $))$;

\% Adjust the lengths of load vectors to account for the

\% removal of bad sandl data points, which leaves 'holes'

$\%$ in the sandl vectors

for $i=1$ :length (load_freqw)

if $i<=$ length (load_freqw)

if sandl_freqw(i) = load_freqw(i)

load_freqw $=[$ load_freqw $(1: i-1) / /$

load_freqw(i+1:length (load_freqw))];

load_data $=[$ load_data $(1: i-1) / /$

load_data(i+1:length(load_data))]; end

end

end

\% Pad the end of the load data to match length of sandl

o data

for $i=$ length (load_freqw) +1 : length (sandl_freqw)

load_data $(i)=$ real (load_data (length (load_freqw) ) $+j * 0$; 


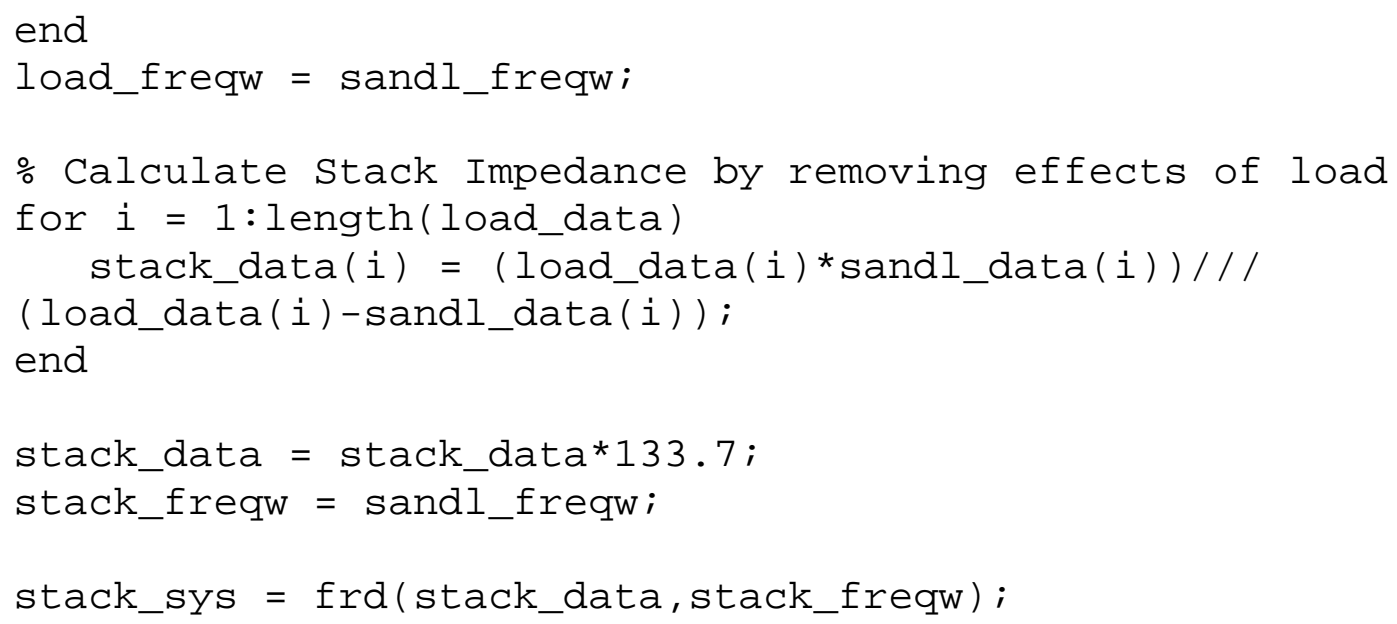




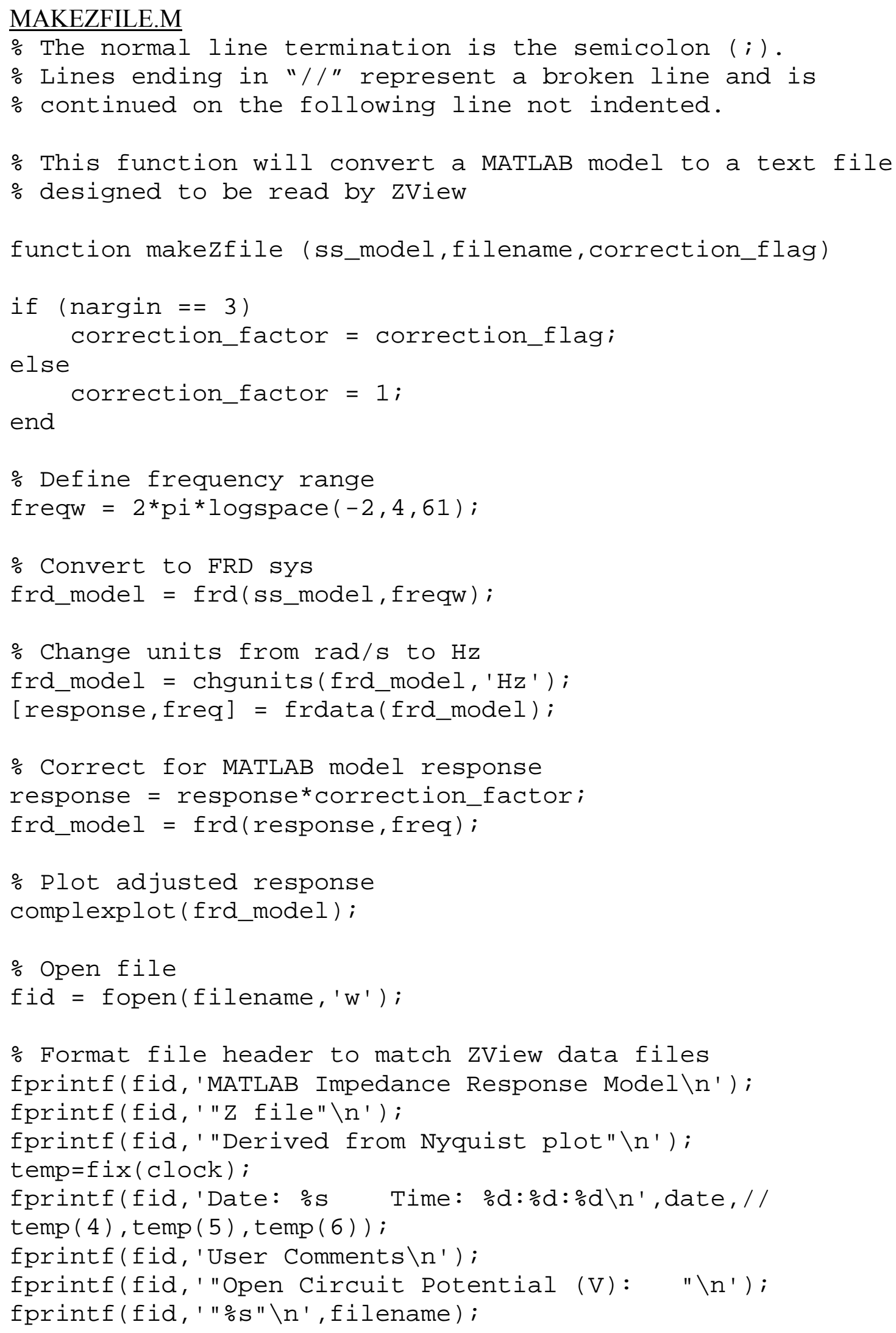


o Save data to file

for $i=1$ :length (freq)

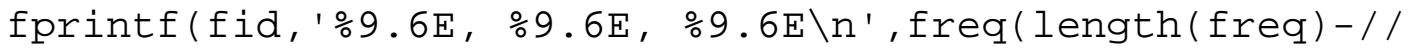

$i+1$ ), real (response (length (freq) -i+1)), imag (response//

(length (freq) $-i+1))$ );

end

fclose (fid); 


\section{COMPLEXPLOT.M}

o The normal line termination is the semicolon ( $;$ ).

o Lines ending in "//" represent a broken line and is

\% continued on the following line not indented.

\% This function will generate a complex plane plot for the given FRD System

function complexplot (frd_sys)

[tempdata, tempfreq] = frdata(frd_sys);

tempdata2 $(1:$ length (tempdata) $)=/ /$

tempdata $(:,:, 1:$ length (tempdata)) ;

tempdata $=$ transpose (tempdata2);

plot (tempdata, '.-') ;

set (gca, 'YDir', 'reverse') ;

axis equal; 


\section{$\underline{\text { SIMULINK MODEL SETUP FILE }}$}

Table A.1: Assignment of Setup File Variables

\begin{tabular}{|c|c|c|}
\hline Parameter & Name & Setup File Variable Name \\
\hline $\mathrm{A}_{\text {cell }}$ & Cell Active Area & CellActiveArea \\
\hline $\mathrm{C}_{\mathrm{DL}}$ & Double Layer Capacitance & SpecificCellCapacitance \\
\hline \multirow{2}{*}{$\mathrm{D}^{\prime}$} & \multirow{2}{*}{ Effective Diffusion Coefficient } & O2EffectiveDiffCoef \\
\hline & & H2EffectiveDiffCoef \\
\hline$D_{H_{2}}$ & Hydrogen Diffusion Coefficient & H2DiffusionCoef \\
\hline$D_{\mathrm{O}_{2}}$ & Oxygen Diffusion Coefficient & O2DiffusionCoef \\
\hline \multirow{2}{*}{$\mathrm{E}_{\mathrm{o}}$} & \multirow{2}{*}{ Standard State Potential } & StandardStateVoltagePotential_A \\
\hline & & StandardStateVoltagePotential_B \\
\hline $\mathrm{F}$ & Faraday's Constant & Faraday \\
\hline$i_{o}$ & Exchange Current Density & ExchangeCurrent \\
\hline$i_{o}^{r e f}$ & Reference Exchange Current Density & RefExchangeCurrent \\
\hline $\mathrm{p}$ & Pressure & Pop \\
\hline$p_{H_{2}}$ & Partial Pressure of Hydrogen & $\mathrm{PH} 2$ \\
\hline$p_{\mathrm{H}_{2}}^{\text {ref }}$ & Reference Partial Pressure of Hydrogen & REF_PH2 \\
\hline$p_{\mathrm{O}_{2}}$ & Partial Pressure of Oxygen & $\mathrm{PO} 2$ \\
\hline$p_{O_{2}}^{r e f}$ & Reference Partial Pressure of Oxygen & REF_PO2 \\
\hline $\mathrm{p}_{\mathrm{o}}$ & Reference Standard State Pressure & Pstd \\
\hline $\mathrm{R}$ & Universal Gas Constant & Runiv \\
\hline $\mathrm{r}_{\text {ohmic }}$ & Area Specific Ohmic Resistance & SpecificCellResistance \\
\hline $\mathrm{T}$ & Temperature & Top \\
\hline \multirow{2}{*}{$\mathrm{T}_{\text {ref }}$} & \multirow{2}{*}{ Reference Temperature } & REF_TH2 \\
\hline & & REF_TO2 \\
\hline$\alpha$ & Transfer Coefficient & TransferCoefficient \\
\hline$\delta$ & Diffusion Layer Thickness & DiffusionLayerThickness \\
\hline$\varepsilon$ & Diffusion Layer Porosity & DiffusionLayerPorosity \\
\hline$\eta$ & Overpotential & eta \\
\hline$\tau$ & Diffusion Layer Tortuosity & DiffusionLayerTortuosity \\
\hline
\end{tabular}




\section{$\underline{\text { SETUP FILE CODE LISTING }}$}

\% The normal line termination is the semicolon (i).

\% Lines ending in "//" represent a broken line and is

o continued on the following line not indented.

ㅇimulink Fuel Cell Model Parameters

$\circ$

\%Written by:

oDr. Randall S. Gemmen

oUSDOE/National Energy Technology Laboratory

\%Morgantown, WV 26507

orandall.gemmen@net 1 .doe.gov

$\circ$

\% Revised by Mark Kim and William W. Clark -- 1-24-02

\% Revised by Randy Gemmen \& Brian Hetzer for enhanced

o diffusion model -- 7-15-02

\% Revised by Brian Hetzer to include Look-Up Table to

\% improve Electochemical Loss -- 9-30-02

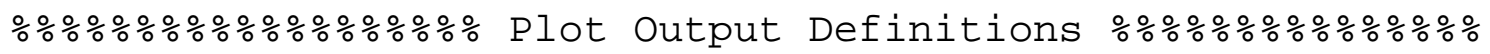
MyNumPlotPoints $=20000$;

MyDecimation=10;

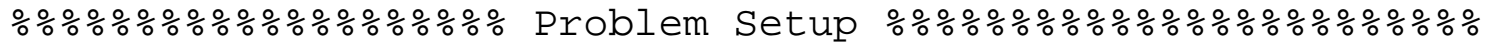

으 Initial Conditions $==>67$ Amps

ICPH2=56310; \%Pa--Initial cell H2 partial pressure

ICPO2=8194; $\%$ Pa--Initial cell O2 partial pressure

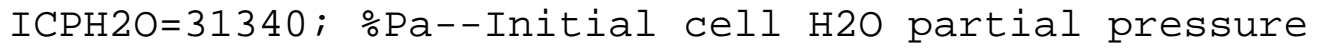

ICElectrochem $=0.1904 ;$ oVolt--Initial electrochemical

o overpotential

○Constants

Prefflow $=101000.0 ; \% \mathrm{~Pa}--$ reference pressure for definition o of standard flows

Trefflow $=273.15+25.0 ; \% \mathrm{~K}--$ reference pressure for definition o of standard flows

Pstd $=100000.0 ; \% \mathrm{~Pa}--$ standard state operating pressure

Tstd $=273.15+25.0$; $\% \mathrm{~K}--$ standard state operating temperature

Faraday $=96439.0 ;$ ocoulomb/gm-mole of e-

Runiv=8.3144; \%joule/gm-mole-K

$\mathrm{MWH} 2 \mathrm{O}=18.0 ; \% \mathrm{gm} / \mathrm{gm}-\mathrm{mol}$

StandardStateVoltagePotential_A=1.229;

StandardStateVoltagePotential_B $=-0.00085$;

SpecificCellResistance=0.2408; \%ohm-cm2 


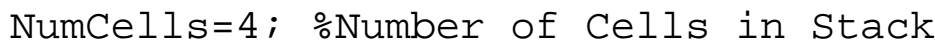

CellActiveWidth=0.1162; m--electrochemically active region \% of cell

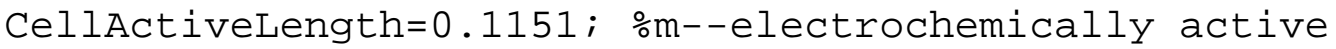
o region of cell

CellActiveArea=CellActiveLength ${ }^{*}$ CellActiveWidth; $\frac{\circ}{\circ}{ }^{2}$

$\mathrm{Pop}=101000.0 ; \% \mathrm{~Pa}-$-operating pressure

TempC $=70.0 ;$ oCelsius--operating temperature

Top $=273.0+\mathrm{TempC} ; \quad \mathrm{K}$--operating temperature

TopHumid=273+TempC; $\% \mathrm{~K}-$-humidifier temperature

LoadResistance $=48.0 / 31.25 / 60.0 *$ NumCells; $\%$ ohm (Load=typical o stack voltage/typical current)

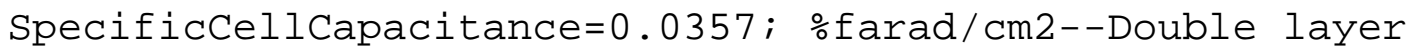
\% capacitance

Define Electrochemical Loss Mechanism

LowCurrentVoltageLoss $=0.2528$; \%volt--this is the loss per \% cell that occurs at the low current

RefExchangeCurrent $=17 ; \circ \mathrm{Amp} / \mathrm{m}^{\wedge} 2$

ExchangeCurrent $=$ RefExchangeCurrent*CellActiveArea; $\%$ Amp TransferCoefficient $=0.5$;

○Create Input and Output vectors for Butler-Volmer Look-Up ㅇ Table

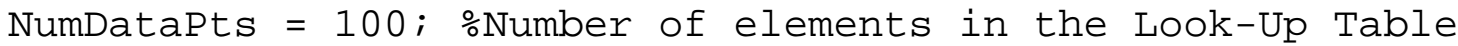
eta_hi $=0.0664$; $\%$ where eta is the Electric Loss due to the o interfacial kinetics.

eta_lo $=0.0071$; $\frac{\circ}{T}$ The $h i$ and lo values are for the ratios \% of current density over exchange current odensity (i/io) $=10.000$ and 0.500 , \% respectively.

eta_step $=$ (eta_hi-eta_lo)/(NumDataPts-1); \%Caculate the \% spacing between each data point

for $i=1:$ NumDatapts

LUT_output $(i)=(i-1)$ *eta_step + eta_lo; \%Look-Up Table temp1 $=\exp \left(\left((2 *\right.\right.$ Faraday $) /\left(\right.$ Runiv $\left.\left.{ }^{*} \mathrm{Top}\right)\right) * / /$ oOutput vector

TransferCoefficient*LUT_output(i)); $\%$ First term of B-V Eq temp $2=\exp (((2 *$ Faraday $) /($ Runiv*Top $)) * / /$

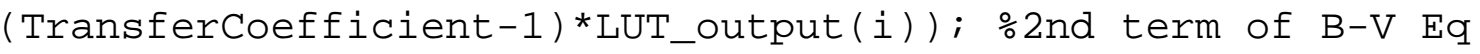
LUT_input $(i)=$ temp1 - temp2; $\quad$ oLUT Input vector end; 
Define diffusion loss parameters

DiffusionLayerThickness $=0.0004 ; \% \mathrm{~m}$

DiffusionLayerporosity $=0.4$;

DiffusionLayerTortuosity $=4$;

Define diffusion parameters for $\mathrm{O} 2$

$\mathrm{REF} \_\mathrm{TO} 2=353.0$; $\% \mathrm{~K}$

$\mathrm{REF} \_\mathrm{PO} 2=101000.0$; $\frac{\mathrm{Pa}}{}$

O2Diffusioncoef $=2.84 e-5 ; \circ \mathrm{m} 2 /$ sec--oxygen molecular

\% diffusion coef in the cathode

o gas mixture at $\mathrm{REF} \_\mathrm{T}$ and $\mathrm{REF} \_\mathrm{P}$

O2EffectiveDiffCoef=O2DiffusionCoef* (Top/REF_TO2)^1.5*//

(REF_PO2/Pop)*DiffusionLayerPorosity ///

DiffusionLayerTortuosity;

Define diffusion parameters for $\mathrm{H} 2$

$\mathrm{REF} \_\mathrm{TH} 2=353.0$; $\% \mathrm{~K}$

REF_PH2=101000.0; $\cong \mathrm{Pa}$

H2DiffusionCoef=1.1e-4; $\frac{\circ}{2} 2 /$ sec--oxygen molecular diffusion

o coef in the cathode gas mixture

\% at REF_T and REF_P

H2EffectiveDiffCoef=H2DiffusionCoef* (Top/REF_TH2)^1.5*//

(REF_PH2/Pop) *DiffusionLayerPorosity ///

DiffusionLayerTortuosity;

oDefine humidity conditions in the humidifier

AnodeRelativeHumid=0.9;

CathodeRelativeHumid=0.9;

Define active channel volumes where electrochemistry

o occurs

AnodeChannelWidth $=0.001587 ; \% \mathrm{~m}$

AnodeChannelHeight $=0.0007938 ; \% \mathrm{~m}$

AnodeChannelLengt $\mathrm{h}=0.343$; $\frac{\mathrm{m}}{}$

CathodeChannelWidth=0.001587; \%m

CathodeChannelHeight $=0.001587$; $\frac{\mathrm{m}}{}$

CathodeChannelLength=AnodeChannelLength; $\% \mathrm{~m}$

Define material volume capacitance for gas flow equations AnodeHumidifierGasVolume $=3.14159 *(0.3)^{\wedge} 2.0 / 4 * 0.2$; \%m3

AnodeInletManifoldWidth=CellActiveWidth; $\% \mathrm{~m}$

AnodeInletManifoldHeight=AnodeChannelHeight ; $\circ \mathrm{m}$

AnodeInletManifoldDept $h=0.015 ; \% m$

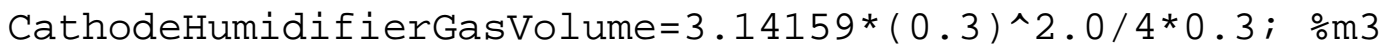

CathodeInletManifoldWidth=CellActiveWidth; $\frac{\mathrm{m}}{}$ 
CathodeInletManifoldHeight=CathodeChannelHeight ; m CathodeInletManifoldDepth=0.015; \%m

oDefine flow equation parameters

$\mathrm{KF}$ actorAnode $=0.00183 ;$ \%LPM $/ \mathrm{PaDeltaP}$

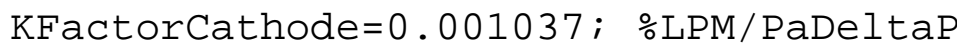

\%Proportional-Integral Control Parameters for Fuel o Utilization Controller

$\mathrm{Ki}=0.05$;

$\mathrm{Kp}=0.1$;

\%Determine mole fractions for anode/cathode inlet-Assume o ideal gas for saturation pressures of $\mathrm{H} 2 \mathrm{O}$. Tref $=273.0+80.0$; $\% \mathrm{~K}--$ Reference Sat Temp for Clapyron eq

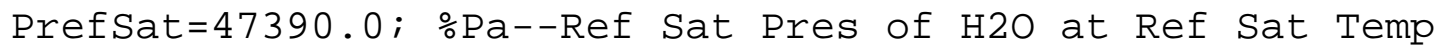
HrefFG $=2308.8 * \mathrm{MWH} 2 \mathrm{O}$; $\frac{\circ}{\mathrm{o}} \mathrm{oule/gm-mol--heat} \mathrm{of} \mathrm{vaporization} \mathrm{at}$ $\div$ Ref Temp.

Define various parameters used in the Simulink program. NumberOfAnodeChannels $=15$;

NumberOfCathodeChannels $=15$;

O2DiffusionCurrentLimit_Param=02EffectiveDiffCoef*4.0*// Faraday/Runiv/Top/DiffusionLayerThickness *CellActiveWidth// *CellActiveLength; \%amp/Pa H2DiffusionCurrentLimit_Param=H2EffectiveDiffCoef*2.0*// Faraday/Runiv/Top/DiffusionLayerThickness *CellActiveWidth// *CellActiveLength; $\%$ amp/Pa

$\mathrm{Kr}=1 / 4.0 /$ Faraday;

CellCapacitance=SpecificCellCapacitance*100*100*//

CellActiveArea; $\%$ Farads

R2fact $=1 / 100.0 / 100.0 /$ CellActiveArea; $。 1 / \mathrm{cm}^{\wedge} 2$

VolAnode = (AnodeChannelWidth*AnodeChannelLength* / /

AnodeChannelHeight *NumberOfAnodeChannels+CellActiveArea * / /

DiffusionLayerThickness*DiffusionLayerPorosity) ; m^3

VolCathode= (CathodeChannelWidth*CathodeChannelLength* / /

CathodeChannelHeight *NumberOfCathodeChannels+//

CellActiveArea*DiffusionLayerThickness*//

DiffusionLayerPorosity) ; ㅇ^3

AnodeInletManifoldVolume=AnodeInletManifoldWidth * / /

AnodeInletManifoldHeight *AnodeInletManifoldDepth; \%m^3 
CathodeInletManifoldVolume=CathodeInletManifoldWidth *// CathodeInletManifoldHeight *CathodeInletManifoldDepth; $\% \mathrm{~m}^{\wedge} 3$

AnodeGasCapacitance=AnodeInletManifoldVolume+//

AnodeHumidifierGasVolume; $\frac{\circ}{\circ}{ }^{3}$

CathodeGasCapacitance=CathodeInletManifoldVolume $+/ /$ CathodeHumidifierGasVolume; $: \mathrm{m}^{\wedge} 3$ 